\title{
The Lanthanide Contraction Revisited
}

\author{
Michael Seitz, Allen G. Oliver, and Kenneth N. Raymond* \\ Department of Chemistry, University of California, \\ Berkeley, CA 94720-1460 \\ E-mail: raymond@socrates.berkeley.edu. Fax: (510) 486-5283.
}

\section{Supporting Information}

Table of Contents:

1. TREN-1,2-HOIQO Lanthanide Complexes

1.1 Structure

1.2 Bond Lengths

1.3 O-O Distances

1.4 Dihedral Angles along the Edges of the Coordination Polyhedron

1.5 Trends in Bond Lengths vs. f Electron Configuration

2. TREN-SAL Lanthanide Complexes

2.1 Structure

2.2 Bond Lengths

2.3 X-X Distances

2.4 Trends in Bond Lengths vs. f Electron Configuration

3. $\left[\mathrm{Ln}(\mathrm{tptz})\left(\mathrm{NO}_{3}\right)_{3}\left(\mathrm{H}_{2} \mathrm{O}\right)\right]$ Complexes

3.1 Structure

3.2 Bond Lengths

3.3 X-X Distances

3.4 Trends in Bond Lengths vs. f Electron Configuration

4. $\left[\mathrm{Ln}(\mathrm{PhMeCH}-\mathrm{DOTAM})\left(\mathrm{H}_{2} \mathrm{O}\right)\right](\mathrm{OTf})_{3}$ Complexes $\mathrm{S} 32$

4.1 Structure

4.2 Bond Lengths

4.3 X-X Distances

4.4 Trends in Bond Lengths vs. $f$ Electron Configuration

5. $\left[\mathrm{Ln}\left(\mathrm{H}_{2} \mathrm{O}\right)_{9}\right]\left(\mathrm{EtOSO}_{3}\right)_{3}$ Complexes

5.1 Bond Lengths

5.2 O-O Distances

5.3 Trends in Bond Lengths vs. f Electron Configuration

6. The Average X-X Distance vs. the Average Ln-X Bond Length

7. References
Page

S2

S2

$\mathrm{S} 2$

$\mathrm{S} 2$

S3

S3

$\mathrm{S} 14$

S14

S14

S15

S15

S19

S19

S19

S20

S20

S32

S33

S33

S44

S44

S44

S45

S48

S51 


\section{TREN-1,2-HOIQO Lanthanide Complexes}

\subsection{Structure}

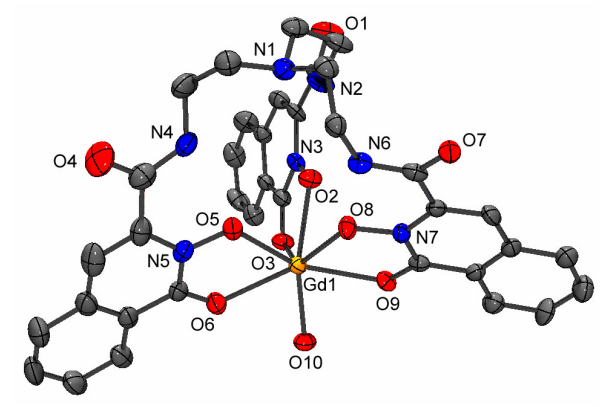

Asymmetric unit of $\left[\mathrm{Gd}(\mathrm{TREN}-1,2-\mathrm{HOIQO})\left(\mathrm{H}_{2} \mathrm{O}\right)\right]^{*} \mathrm{H}_{2} \mathrm{O}$. Thermal ellipsoid plot (Ortep 3 for Windows, ${ }^{1} 50 \%$ probability level) with atom numbering scheme. Hydrogens and the isolated water molecule omitted for clarity.

\subsection{Bond Lengths}

Table S1. Bond lengths in [Ln(TREN-1,2-HOIQO) $\left.\left(\mathrm{H}_{2} \mathrm{O}\right)\right]^{\star} \mathrm{H}_{2} \mathrm{O}$.

\begin{tabular}{|c|c|c|c|c|c|c|c|c|c|}
\hline f electrons (Ln) & $\mathrm{d}_{\mathrm{Ln}-\mathrm{O} 3}(\mathrm{su})[\hat{\mathrm{A}}]$ & $\mathrm{d}_{\mathrm{Ln}-\mathrm{OG}}(\mathrm{su})[\hat{\mathrm{A}}]$ & $\mathrm{d}_{\mathrm{Ln}-\mathrm{Og}}(\mathrm{su})[\overline{\mathrm{A}}]$ & $\mathrm{d}_{\mathrm{Ln}-\mathrm{O} 2}(\mathrm{su})[\hat{\mathrm{A}}]$ & $\mathrm{d}_{\mathrm{Ln}-\mathrm{O} 5}(\mathrm{su})[\hat{\mathrm{A}}]$ & $\mathrm{d}_{\mathrm{Ln}-\mathrm{O} 8}(\mathrm{su})[\hat{\mathrm{A}}]$ & $\mathrm{d}_{\mathrm{Ln}-\mathrm{O} 10}(\mathrm{su})[\hat{\mathrm{A}}]$ & $\mathrm{d}_{\mathrm{Ln}-\mathrm{O} 7}(\mathrm{su})[\hat{\mathrm{A}}]$ & $\Sigma \mathrm{d}_{\mathrm{Ln}-\mathrm{O}}(\mathrm{su})[\AA]$ \\
\hline $0(\mathrm{La})$ & $2.482(4)$ & $2.463(4)$ & $2.479(4)$ & $2.447(4)$ & $2.443(5)$ & $2.473(4)$ & $2.581(4)$ & $2.516(4)$ & $19.884(34)$ \\
\hline $1(\mathrm{Ce})^{2}$ & $2.469(4)$ & $2.445(4)$ & $2.450(4)$ & $2.432(4)$ & $2.426(4)$ & $2.445(4)$ & $2.573(4)$ & $2.478(4)$ & $19.718(32)$ \\
\hline $2(\mathrm{Pr})$ & $2.452(4)$ & $2.427(4)$ & $2.428(4)$ & $2.407(4)$ & $2.405(4)$ & $2.437(3)$ & $2.556(4)$ & $2.473(4)$ & $19.585(31)$ \\
\hline $3(\mathrm{Nd})$ & $2.434(4)$ & $2.397(4)$ & $2.415(4)$ & $2.395(4)$ & 2.394(4) & $2.418(4)$ & $2.536(4)$ & $2.461(4)$ & $19.450(32)$ \\
\hline $4(\mathrm{Pm})$ & - & - & - & - & - & - & - & - & - \\
\hline $5(\mathrm{Sm})$ & $2.396(4)$ & $2.381(4)$ & $2.374(4)$ & $2.377(4)$ & $2.377(4)$ & $2.396(4)$ & $2.518(4)$ & $2.434(4)$ & $19.253(32)$ \\
\hline $6(\mathrm{Eu})$ & $2.384(5)$ & $2.368(5)$ & $2.356(5)$ & $2.361(5)$ & $2.367(5)$ & $2.386(5)$ & $2.501(5)$ & $2.420(5)$ & $19.143(40)$ \\
\hline $7(\mathrm{Gd})$ & $2.373(5)$ & $2.357(5)$ & $2.349(5)$ & $2.352(4)$ & $2.343(5)$ & $2.376(4)$ & $2.491(5)$ & $2.403(5)$ & $19.044(38)$ \\
\hline $8(\mathrm{~Tb})$ & $2.356(4)$ & $2.338(4)$ & $2.333(4)$ & $2.336(4)$ & $2.332(4)$ & $2.361(4)$ & $2.462(4)$ & $2.381(4)$ & $18.899(32)$ \\
\hline 9 (Dy) & $2.340(4)$ & $2.335(4)$ & $2.322(4)$ & $2.329(4)$ & $2.327(4)$ & $2.355(4)$ & $2.460(4)$ & $2.375(4)$ & $18.843(32)$ \\
\hline $10(\mathrm{Ho})$ & $2.327(5)$ & $2.318(5)$ & $2.313(5)$ & $2.312(5)$ & $2.311(5)$ & $2.333(5)$ & $2.456(5)$ & $2.353(5)$ & $18.723(40)$ \\
\hline $11(\mathrm{Er})$ & $2.314(4)$ & $2.309(5)$ & $2.307(4)$ & $2.307(4)$ & $2.313(4)$ & $2.330(4)$ & $2.442(5)$ & $2.353(4)$ & $18.675(34)$ \\
\hline $12(\mathrm{Tm})$ & $2.308(4)$ & $2.300(4)$ & 2.294(4) & $2.297(4)$ & $2.304(4)$ & $2.324(4)$ & $2.424(4)$ & $2.338(4)$ & $18.589(32)$ \\
\hline $13(\mathrm{Yb})$ & $2.277(6)$ & $2.282(6)$ & $2.265(6)$ & $2.300(6)$ & $2.286(6)$ & $2.301(6)$ & $2.437(6)$ & $2.333(6)$ & $18.481(48)$ \\
\hline $14(\mathrm{Lu})$ & $2.288(3)$ & $2.292(3)$ & $2.282(3)$ & $2.294(3)$ & $2.287(3)$ & $2.320(3)$ & $2.418(3)$ & $2.329(3)$ & $18.510(24)$ \\
\hline$\overline{\mathrm{d}_{\mathrm{La}} / \mathrm{d}_{\mathrm{Lu}}}$ & 1.085 & 1.075 & 1.086 & 1.067 & 1.068 & 1.066 & 1.067 & 1.080 & 1.074 \\
\hline
\end{tabular}

\subsection{O-O Distances}

Table S2. Distances O-O (O bound to $\mathrm{Ln})$ in $\left[\mathrm{Ln}(\mathrm{TREN}-1,2-\mathrm{HOIQO})\left(\mathrm{H}_{2} \mathrm{O}\right)\right]^{\star} \mathrm{H}_{2} \mathrm{O}$.

\begin{tabular}{|c|c|c|c|c|c|c|c|c|c|c|c|c|c|c|c|c|c|c|c|}
\hline $\begin{array}{c}\text { f electrons } \\
\text { (Ln) }\end{array}$ & $\begin{array}{c}\text { do7-09 } \\
{[A ̊]}\end{array}$ & $\begin{array}{c}\mathrm{d}_{\text {○3-05 }} \\
{[\AA \AA]}\end{array}$ & $\begin{array}{c}\mathrm{d}_{07-05} \\
{[\hat{\mathrm{A}}]}\end{array}$ & $\begin{array}{c}\mathrm{d}_{\text {O3-09 }} \\
{[\hat{A}]}\end{array}$ & $\begin{array}{c}d_{05-06} \\
{[\AA \AA A}\end{array}$ & 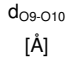 & $\begin{array}{c}\mathrm{d}_{\mathrm{O} 2-03} \\
{[\hat{\mathrm{A}}]}\end{array}$ & $\begin{array}{c}d_{07-08} \\
{[\AA \AA A}\end{array}$ & $\begin{array}{c}\mathrm{d}_{07-010} \\
{[\hat{\mathrm{A}}]}\end{array}$ & $\begin{array}{c}d_{03-06} \\
{[\hat{A}]}\end{array}$ & $\begin{array}{c}d_{07-06} \\
[\AA]]\end{array}$ & $\begin{array}{c}\mathrm{d}_{010-03} \\
{[\AA \AA]}\end{array}$ & $\begin{array}{c}d_{02-05} \\
{[\AA \AA A]}\end{array}$ & $\begin{array}{c}\mathrm{d}_{\mathrm{O2}-09} \\
{[\hat{\mathrm{A}}]}\end{array}$ & $\begin{array}{c}\mathrm{d}_{09-08} \\
{[\hat{A}]}\end{array}$ & $\begin{array}{c}d_{08-05} \\
{[\AA \AA]}\end{array}$ & $\begin{array}{c}\mathrm{d}_{06-010} \\
{[\hat{A}]}\end{array}$ & $\begin{array}{c}\mathrm{d}_{02-08} \\
{[\hat{A}]}\end{array}$ & $\begin{array}{c}\Sigma \mathrm{d}_{\mathrm{O}-\mathrm{O}} \\
[\AA]]\end{array}$ \\
\hline 0 (La) & 3.120 & 3.942 & 3.408 & 3.960 & 2.568 & 3.213 & 2.557 & 3.078 & 3.124 & 3.284 & 3.143 & 3.092 & 3.158 & 3.602 & 2.576 & 3.090 & 2.905 & 2.915 & 56.735 \\
\hline $1(\mathrm{Ce})^{2}$ & 3.109 & 3.902 & 3.379 & 3.912 & 2.555 & 3.159 & 2.562 & 3.043 & 3.104 & 3.224 & 3.122 & 3.064 & 3.128 & 3.555 & 2.574 & 3.065 & 2.907 & 2.881 & 56.245 \\
\hline $2(\operatorname{Pr})$ & 3.112 & 3.908 & 3.353 & 3.840 & 2.557 & 3.119 & 2.551 & 3.039 & 3.086 & 3.217 & 3.100 & 3.023 & 3.106 & 3.509 & 2.576 & 3.031 & 2.884 & 2.862 & 55.873 \\
\hline $3(\mathrm{Nd})$ & 3.112 & 3.897 & 3.335 & 3.793 & 2.551 & 3.086 & 2.549 & 3.006 & 3.072 & 3.190 & 3.077 & 2.992 & 3.086 & 3.477 & 2.576 & 2.997 & 2.856 & 2.843 & 55.495 \\
\hline $4(\mathrm{Pm})$ & - & - & - & - & - & - & & & & & & & & & & & & & \\
\hline $5(\mathrm{Sm})$ & 3.085 & 3.865 & 3.319 & 3.703 & 2.562 & 3.023 & 2.567 & 2.986 & 3.040 & 3.135 & 3.054 & 2.931 & 3.040 & 3.428 & 2.573 & 2.959 & 2.839 & 2.795 & 54.904 \\
\hline $6(\mathrm{Eu})$ & 3.083 & 3.866 & 3.294 & 3.646 & 2.560 & 2.976 & 2.551 & 2.967 & 3.031 & 3.137 & 3.038 & 2.910 & 3.023 & 3.392 & 2.570 & 2.940 & 2.838 & 2.775 & 54.597 \\
\hline 7 (Gd) & 3.085 & 3.852 & 3.258 & 3.610 & 2.562 & 2.973 & 2.546 & 2.953 & 3.015 & 3.120 & 3.023 & 2.888 & 3.012 & 3.363 & 2.565 & 2.914 & 2.804 & 2.758 & 54.301 \\
\hline $8(\mathrm{~Tb})$ & 3.067 & 3.823 & 3.253 & 3.578 & 2.556 & 2.936 & 2.534 & 2.928 & 2.976 & 3.074 & 3.004 & 2.850 & 2.995 & 3.326 & 2.567 & 2.890 & 2.774 & 2.749 & 53.880 \\
\hline 9 (Dy) & 3.074 & 3.817 & 3.237 & 3.535 & 2.558 & 2.901 & 2.544 & 2.918 & 2.969 & 3.063 & 3.001 & 2.842 & 2.984 & 3.302 & 2.570 & 2.881 & 2.788 & 2.724 & 53.708 \\
\hline $10(\mathrm{Ho})$ & 3.061 & 3.795 & 3.208 & 3.515 & 2.552 & 2.891 & 2.539 & 2.884 & 2.959 & 3.033 & 2.977 & 2.826 & 2.955 & 3.284 & 2.558 & 2.855 & 2.767 & 2.702 & 53.361 \\
\hline $11(\mathrm{Er})$ & 3.060 & 3.792 & 3.211 & 3.489 & 2.544 & 2.876 & 2.549 & 2.884 & 2.948 & 3.018 & 2.976 & 2.802 & 2.953 & 3.272 & 2.564 & 2.853 & 2.753 & 2.684 & 53.228 \\
\hline $12(\mathrm{Tm})$ & 3.059 & 3.775 & 3.217 & 3.470 & 2.562 & 2.854 & 2.538 & 2.881 & 2.924 & 2.998 & 2.966 & 2.792 & 2.933 & 3.248 & 2.572 & 2.835 & 2.736 & 2.682 & 53.042 \\
\hline $13(\mathrm{Yb})$ & 3.042 & 3.738 & 3.188 & 3.412 & 2.542 & 2.824 & 2.521 & 2.856 & 2.920 & 2.958 & 2.954 & 2.788 & 2.927 & 3.221 & 2.544 & 2.809 & 2.741 & 2.669 & 52.654 \\
\hline $14(\mathrm{Lu})$ & 3.054 & 3.752 & 3.194 & 3.417 & 2.552 & 2.831 & 2.535 & 2.860 & 2.913 & 2.974 & 2.956 & 2.761 & 2.928 & 3.216 & 2.579 & 2.812 & 2.722 & 2.674 & 52.730 \\
\hline $\mathrm{d}_{\mathrm{La}} / \mathrm{d}_{\mathrm{Lu}}$ & 1.022 & 1.051 & 1.067 & 1.159 & 1.006 & 1.135 & 1.009 & 1.076 & 1.072 & 1.104 & 1.063 & 1.120 & 1.079 & 1.120 & 0.999 & 1.090 & 1.067 & 1.090 & 1.076 \\
\hline
\end{tabular}




\subsection{Dihedral Angles along the Edges of the Coordination Polyhedron}

Table S3. Dihedral angles along the edges of the coordination polyhedron [ ${ }^{\circ}$. Shape measure $\mathrm{SM}_{\text {tdh }}$ (deviation relative to an ideal trigonaldodecahedron) and $\mathrm{SM}_{\mathrm{Gd}}$ (deviation relative to the gadolinium complex). ${ }^{3}$

\begin{tabular}{|c|c|c|c|c|c|c|c|c|c|c|c|c|c|c|c|}
\hline edge & ideal & La & $\mathrm{Ce}^{2}$ & $\mathrm{Pr}$ & $\mathrm{Nd}$ & $\mathrm{Sm}$ & Eu & Gd & $\mathrm{Tb}$ & Dy & Ho & $\mathrm{Er}$ & $\mathrm{Tm}$ & $\mathrm{Yb}$ & Lu \\
\hline 07-08 & 74.3 & 55.07 & 55.68 & 56.25 & 56.68 & 57.31 & 57.75 & 57.94 & 58.37 & 58.77 & 58.70 & 58.85 & 59.35 & 59.72 & 60.10 \\
\hline O7-O5 & 29.9 & 41.41 & 41.56 & 41.77 & 42.10 & 42.00 & 42.24 & 42.42 & 42.09 & 42.36 & 42.54 & 42.75 & 42.31 & 43.00 & 41.60 \\
\hline O7-06 & 61.5 & 49.04 & 48.85 & 49.16 & 49.02 & 49.25 & 49.54 & 49.40 & 49.45 & 49.51 & 49.18 & 49.02 & 49.47 & 48.91 & 50.19 \\
\hline O7-010 & 61.5 & 56.74 & 57.09 & 57.40 & 57.55 & 57.88 & 57.89 & 57.86 & 57.90 & 58.21 & 58.17 & 58.30 & 58.38 & 59.55 & 57.81 \\
\hline O7-09 & 29.9 & 47.45 & 46.36 & 45.77 & 45.51 & 44.62 & 43.87 & 43.60 & 43.44 & 42.49 & 42.23 & 42.41 & 42.03 & 40.98 & 41.47 \\
\hline O6-O10 & 53.2 & 61.63 & 61.31 & 61.04 & 60.82 & 60.79 & 60.78 & 61.22 & 60.70 & 60.74 & 60.98 & 60.80 & 60.54 & 60.87 & 61.31 \\
\hline O9-O10 & 74.3 & 68.17 & 69.12 & 68.39 & 68.26 & 68.38 & 68.31 & 68.07 & 67.95 & 68.35 & 68.79 & 68.42 & 68.37 & 69.03 & 68.33 \\
\hline O3-O9 & 29.9 & 30.36 & 29.78 & 30.81 & 31.32 & 31.28 & 31.93 & 32.46 & 32.32 & 32.54 & 32.67 & 33.06 & 33.07 & 31.99 & 33.02 \\
\hline O2-O9 & 61.5 & 40.79 & 41.52 & 40.91 & 41.39 & 41.47 & 40.91 & 41.13 & 42.01 & 42.00 & 42.25 & 42.33 & 42.69 & 43.26 & 43.33 \\
\hline O2-O8 & 53.2 & 63.81 & 63.67 & 63.64 & 63.10 & 63.33 & 63.25 & 63.29 & 62.81 & 62.97 & 62.61 & 62.80 & 62.40 & 62.70 & 62.62 \\
\hline O2-O5 & 61.5 & 62.25 & 62.23 & 62.55 & 62.98 & 63.87 & 64.15 & 64.18 & 63.98 & 64.33 & 64.58 & 64.75 & 64.47 & 65.26 & 64.65 \\
\hline O3-O5 & 29.9 & 13.68 & 13.98 & 13.05 & 13.10 & 12.47 & 12.02 & 11.46 & 11.93 & 11.56 & 11.64 & 11.57 & 12.06 & 11.18 & 11.71 \\
\hline O2-O3 & 74.3 & 86.69 & 87.41 & 87.47 & 87.83 & 88.45 & 88.63 & 88.64 & 89.19 & 89.63 & 89.89 & 89.75 & 89.33 & 89.71 & 89.14 \\
\hline O5-O6 & 74.3 & 80.45 & 80.35 & 80.54 & 80.37 & 80.85 & 81.14 & 81.17 & 81.17 & 81.44 & 81.28 & 81.53 & 81.25 & 81.25 & 81.69 \\
\hline O3-O6 & 53.2 & 57.05 & 57.54 & 57.88 & 57.78 & 58.11 & 58.46 & 58.73 & 59.02 & 59.53 & 59.43 & 59.37 & 59.66 & 59.67 & 59.85 \\
\hline O10-O3 & 74.3 & 72.70 & 72.39 & 72.08 & 71.85 & 71.41 & 71.10 & 70.54 & 70.02 & 69.50 & 69.50 & 69.21 & 68.65 & 68.80 & 67.86 \\
\hline O9-08 & 61.5 & 68.95 & 68.61 & 68.48 & 67.87 & 67.32 & 67.16 & 67.06 & 66.82 & 66.48 & 66.52 & 66.14 & 66.11 & 65.35 & 65.52 \\
\hline O8-O5 & 61.5 & 57.52 & 57.45 & 57.87 & 57.78 & 57.83 & 57.87 & 57.83 & 58.56 & 58.19 & 58.03 & 57.95 & 58.62 & 57.91 & 58.68 \\
\hline $\mathrm{SM}_{\mathrm{tdh}}$ & 0 & 11.01 & 10.77 & 10.81 & 10.70 & 10.66 & 10.70 & 10.77 & 10.56 & 10.58 & 10.57 & 10.64 & 10.44 & 10.41 & 10.34 \\
\hline $\mathrm{SM}_{\mathrm{Gd}}$ & - & 2.01 & 1.78 & 1.44 & 1.24 & 0.89 & 0.70 & 0 & 0.65 & 0.69 & 0.72 & 0.74 & 0.94 & 1.27 & 1.26 \\
\hline
\end{tabular}

\subsection{Trends in Bond Lengths and O-O Distances vs. $f$ Electron Configuration}

Bond lengths:

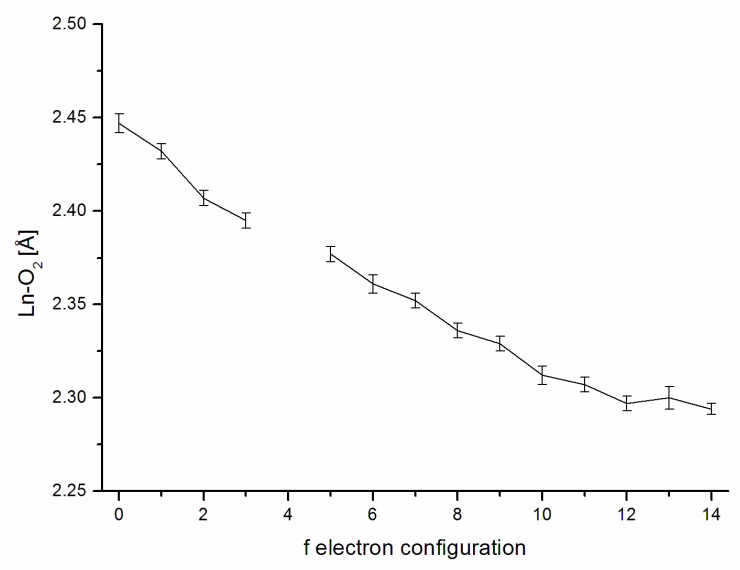

Figure S1. Bond length Ln-O2 vs. f electronic configuration. 


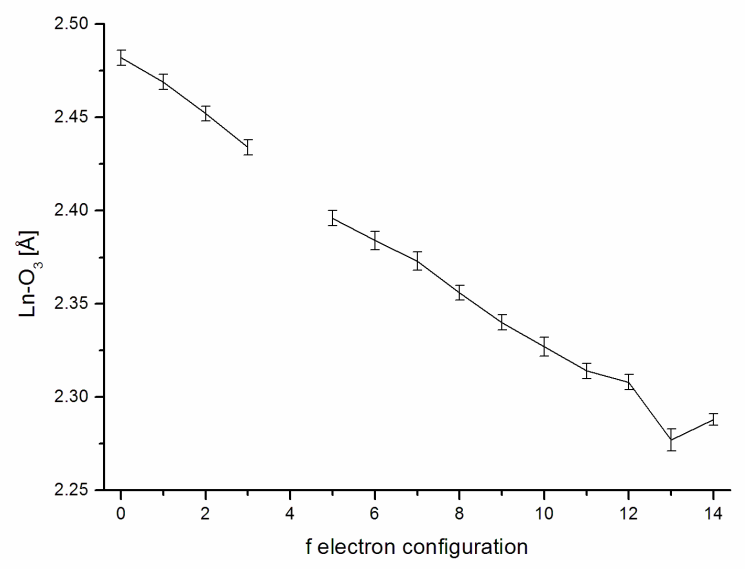

Figure S2. Bond length Ln-O3 vs. f electronic configuration.

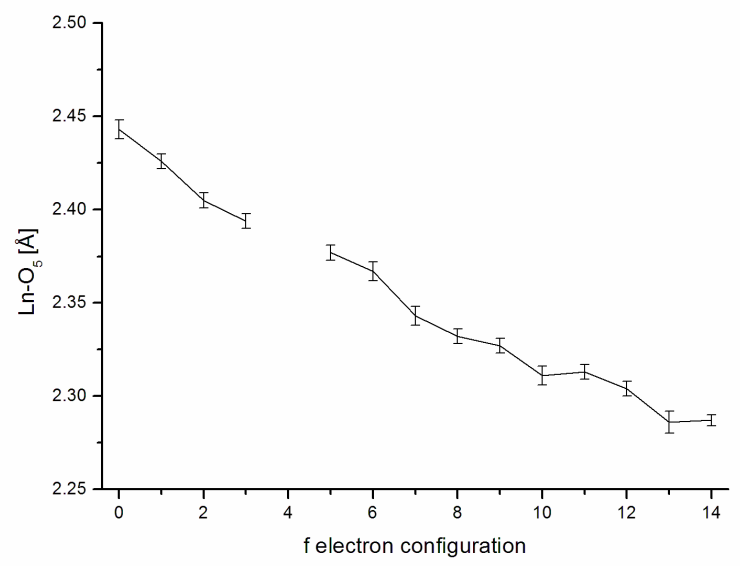

Figure S3. Bond length Ln-O5 vs. f electronic configuration.

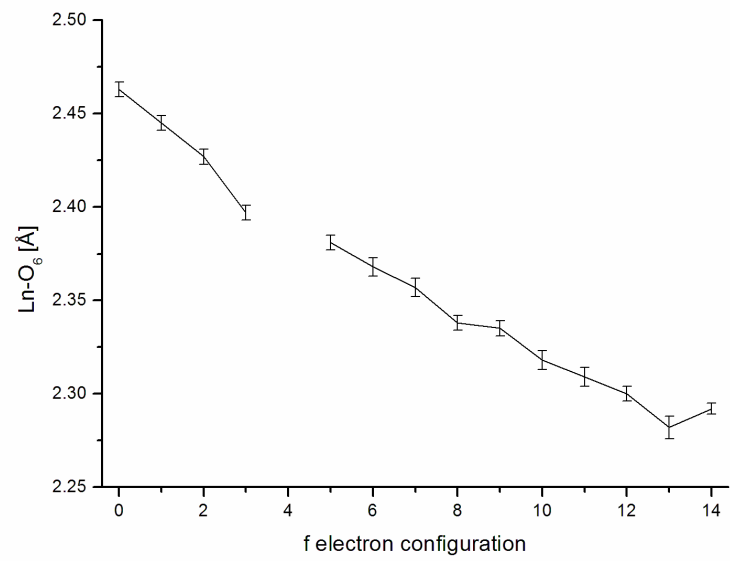

Figure S4. Bond length Ln-O6 vs. f electronic configuration. 


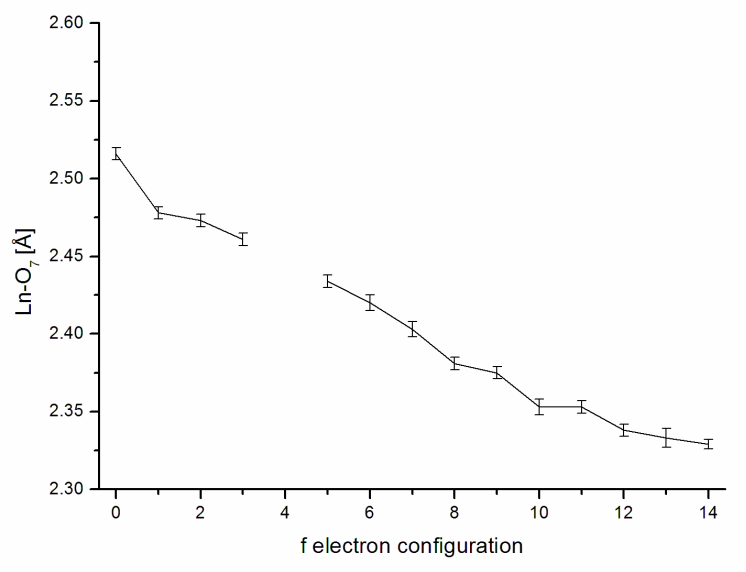

Figure S5. Bond length Ln-O7 vs. f electronic configuration.

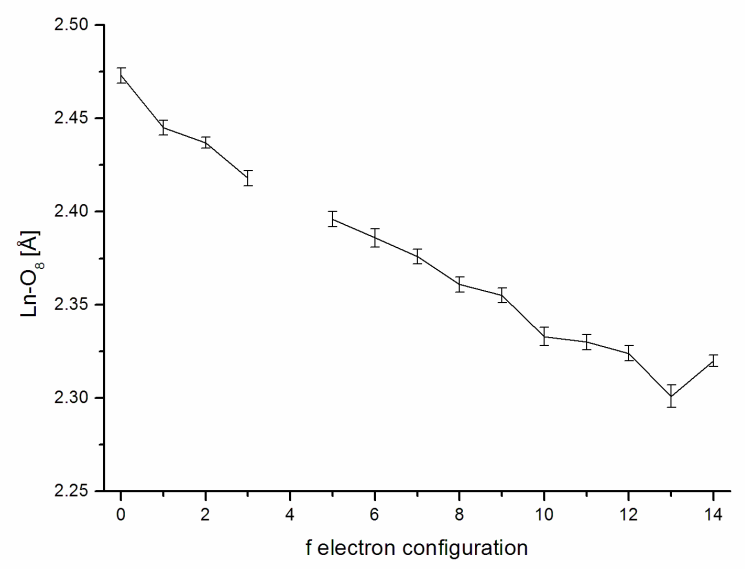

Figure S6. Bond length Ln-O8 vs. f electronic configuration.

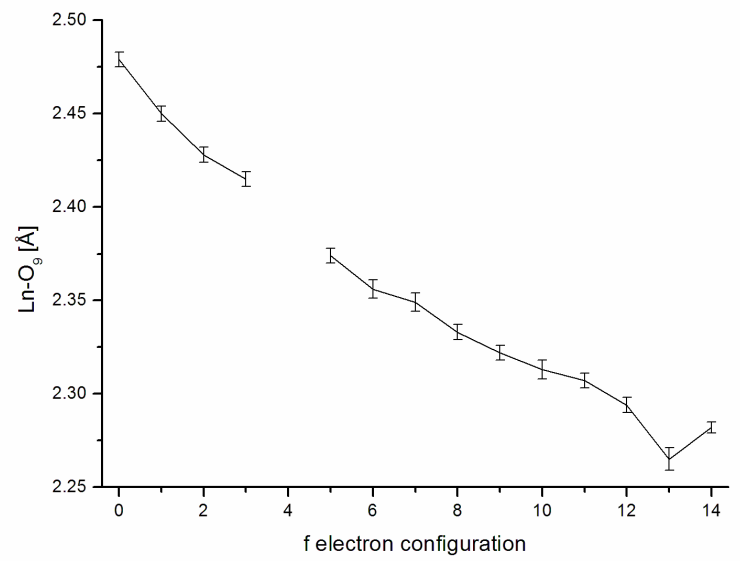

Figure S7. Bond length Ln-O9 vs. f electronic configuration. 


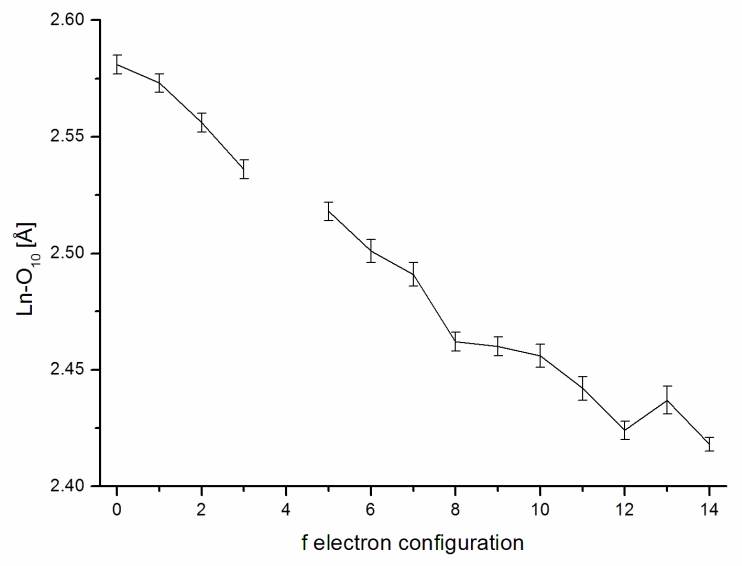

Figure S8. Bond length Ln-O10 vs. f electronic configuration.

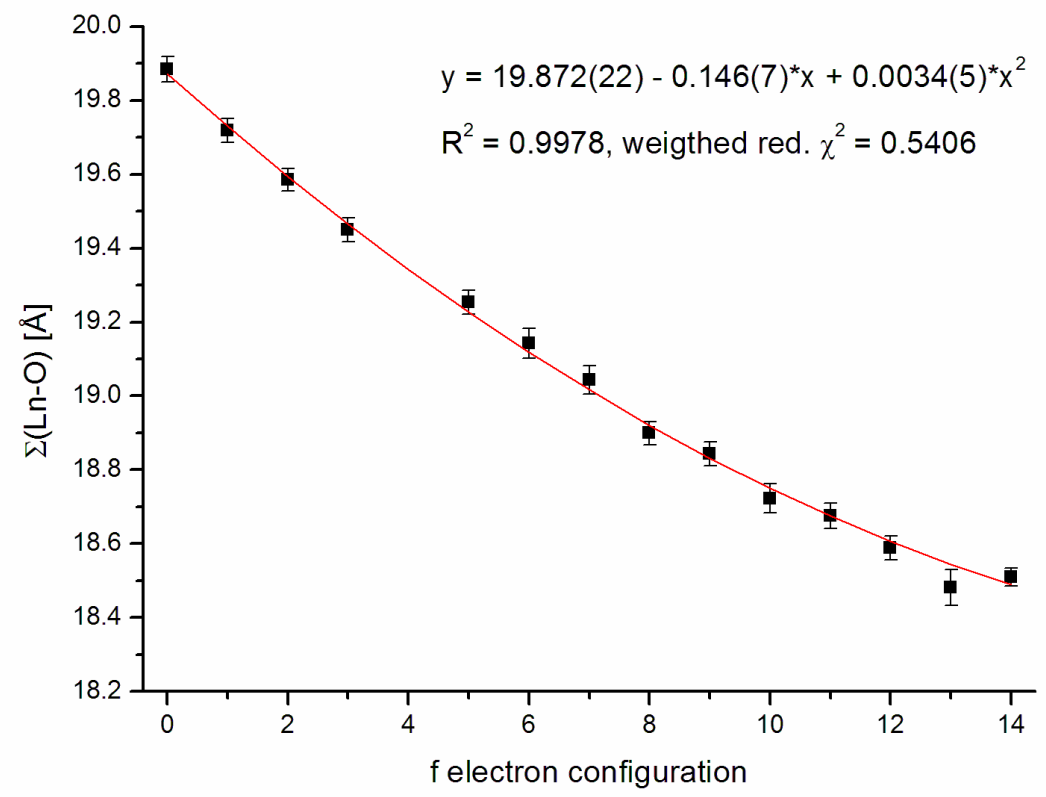

Figure S9. Sum of bond lengths Ln-O vs. $f$ electronic configuration. Quadratic fit $\left(\chi^{2}-\right.$ weighting factor: $\mathrm{su}^{-2}$ ). 
Distances O-O:

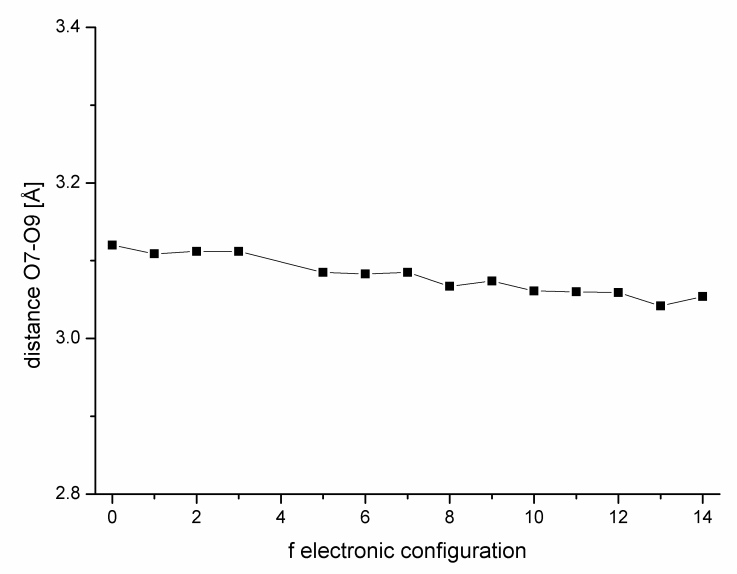

Figure S10. Distance O7-O9 vs. f electronic configuration.

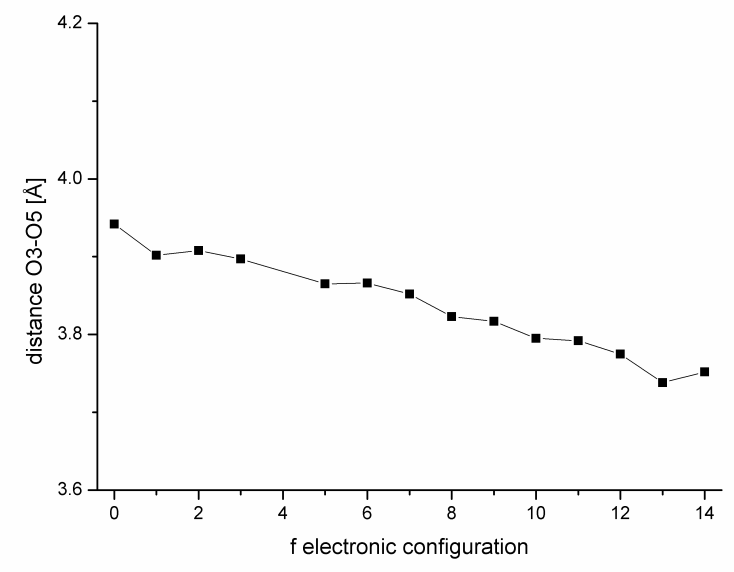

Figure S11. Distance O3-O5 vs. f electronic configuration.

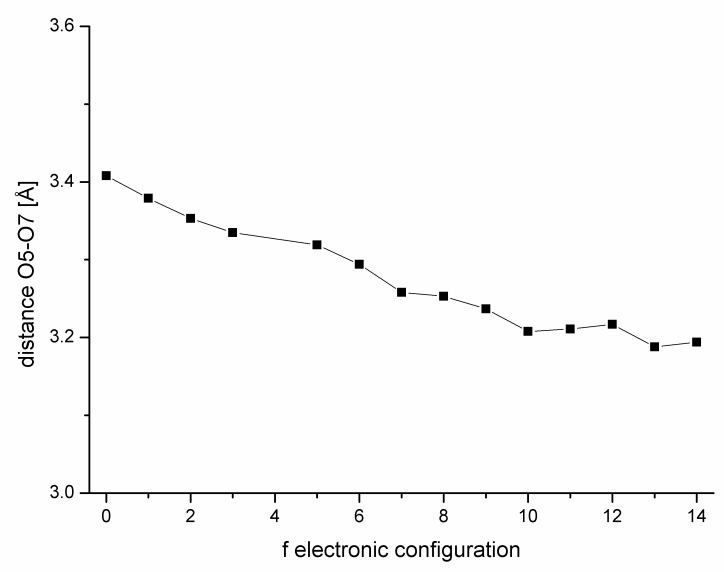

Figure S12. Distance O7-O5 vs. f electronic configuration. 


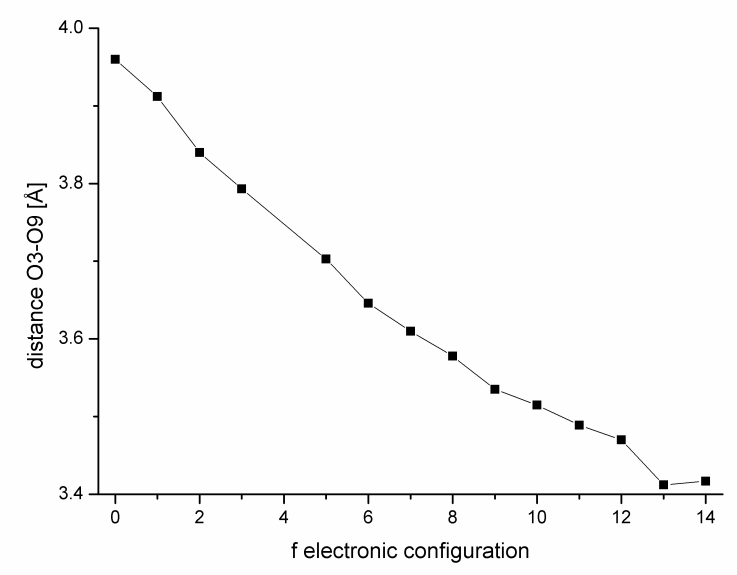

Figure S13. Distance O3-O9 vs. f electronic configuration.

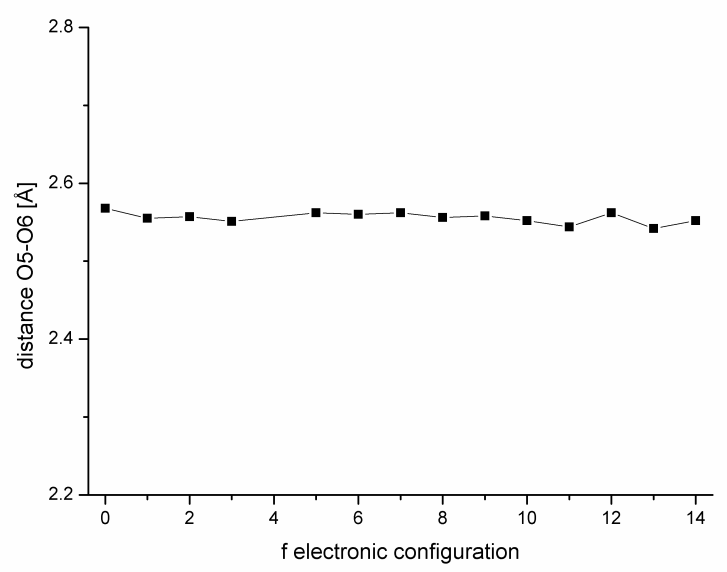

Figure S14. Distance O5-O6 vs. f electronic configuration.

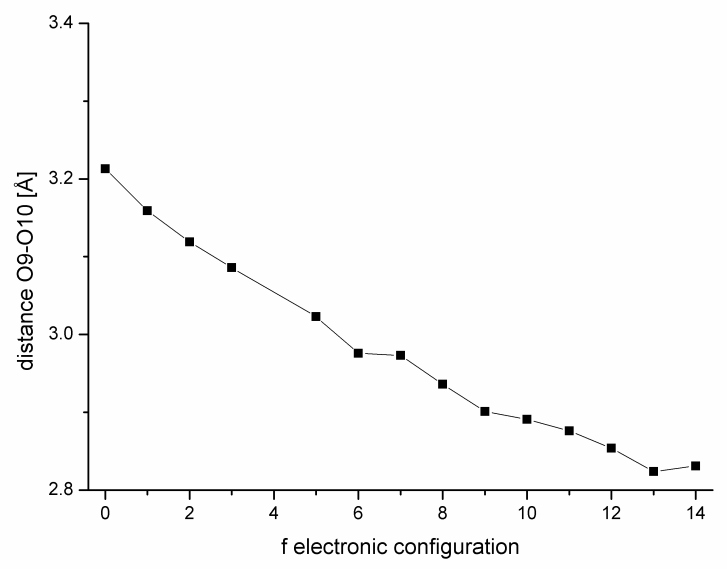

Figure S15. Distance O9-O10 vs. f electronic configuration. 


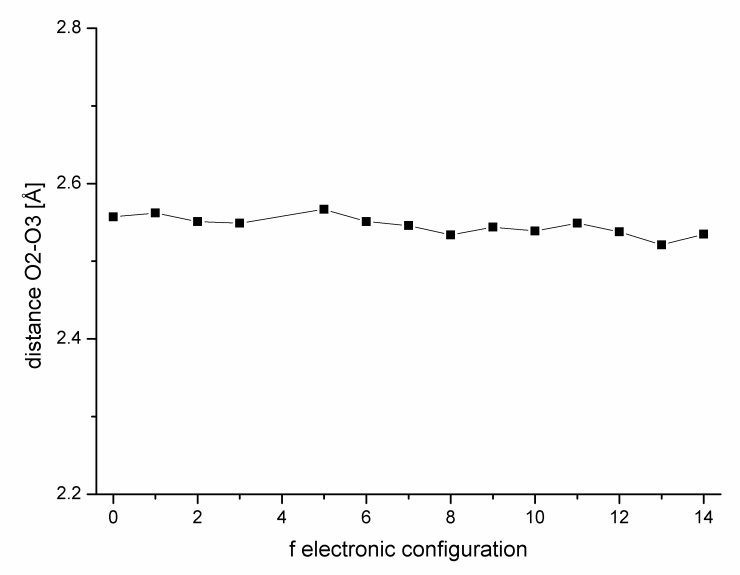

Figure S16. Distance O2-O3 vs. f electronic configuration.

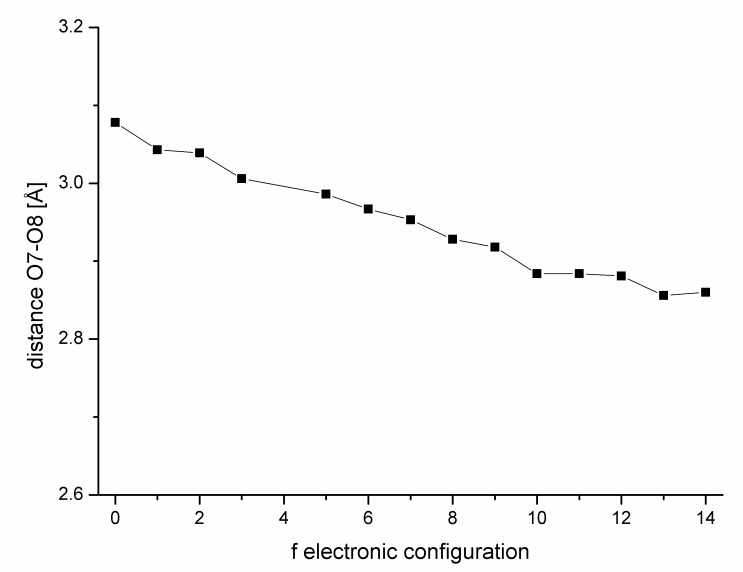

Figure S17. Distance O7-O8 vs. f electronic configuration.

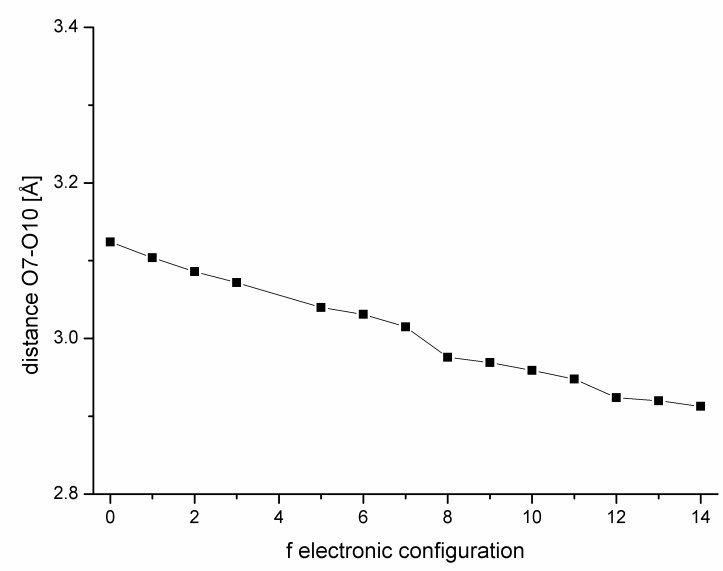

Figure S18. Distance O7-O10 vs. f electronic configuration. 


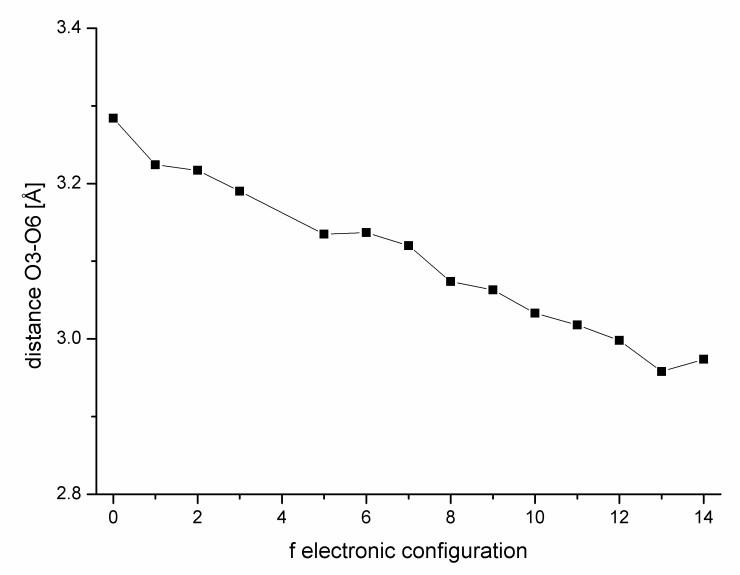

Figure S19. Distance O3-O6 vs. f electronic configuration.

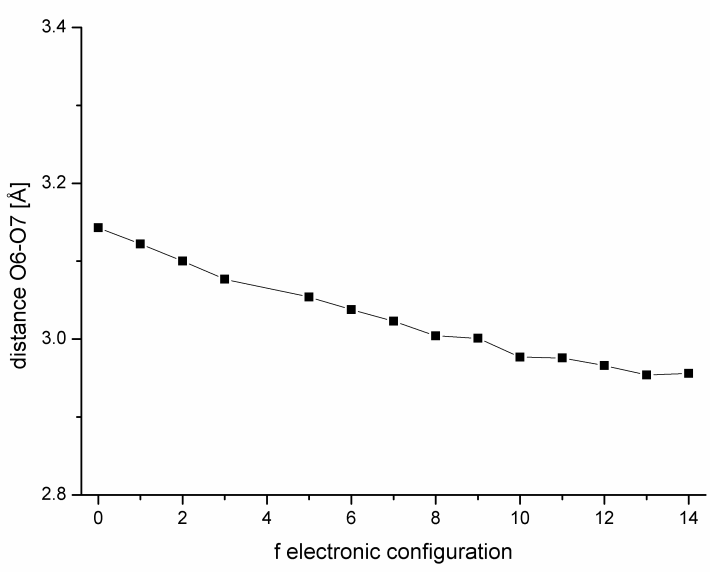

Figure S20. Distance 07-O6 vs. f electronic configuration.

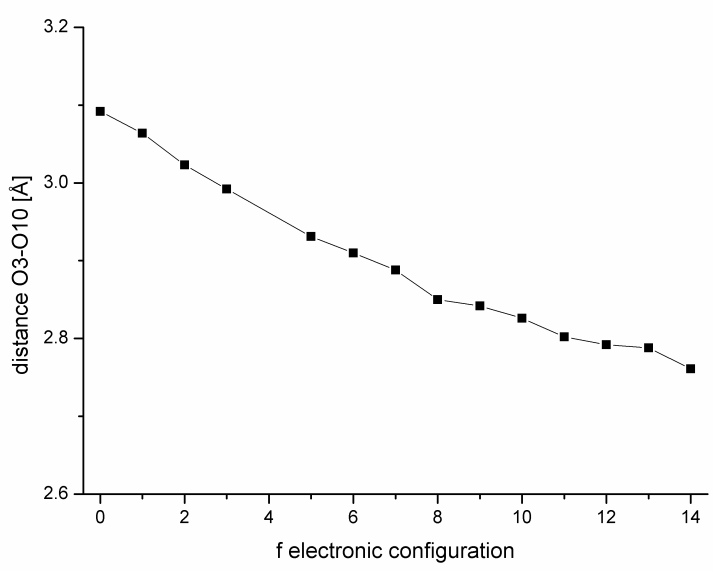

Figure S21. Distance O10-O3 vs. f electronic configuration. 


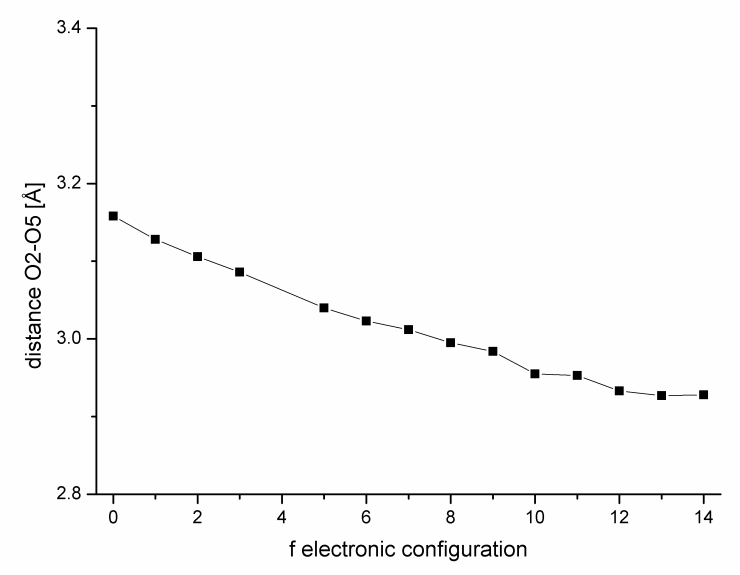

Figure S22. Distance O2-O5 vs. f electronic configuration.

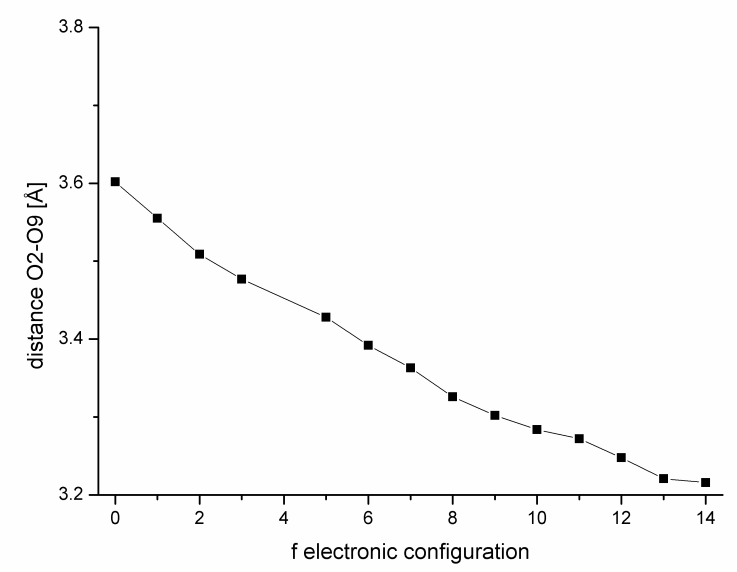

Figure S23. Distance O2-O9 vs. f electronic configuration.

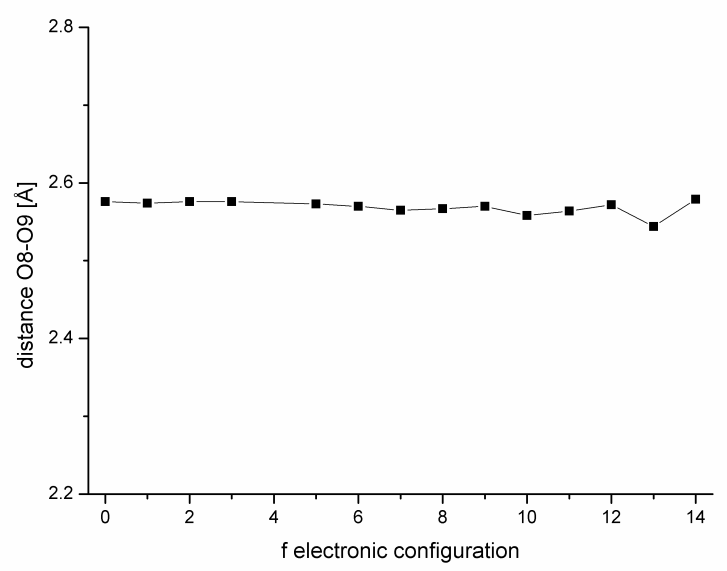

Figure S24. Distance O8-O9 vs. f electronic configuration. 


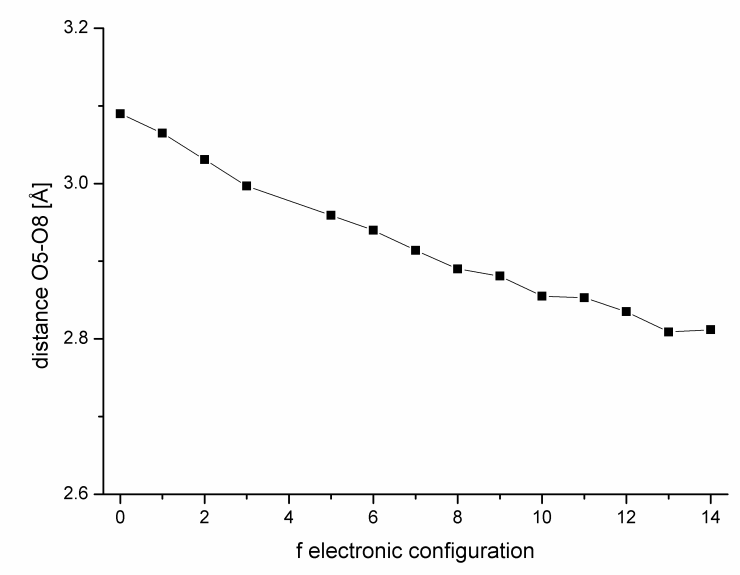

Figure S25. Distance O8-O5 vs. f electronic configuration.

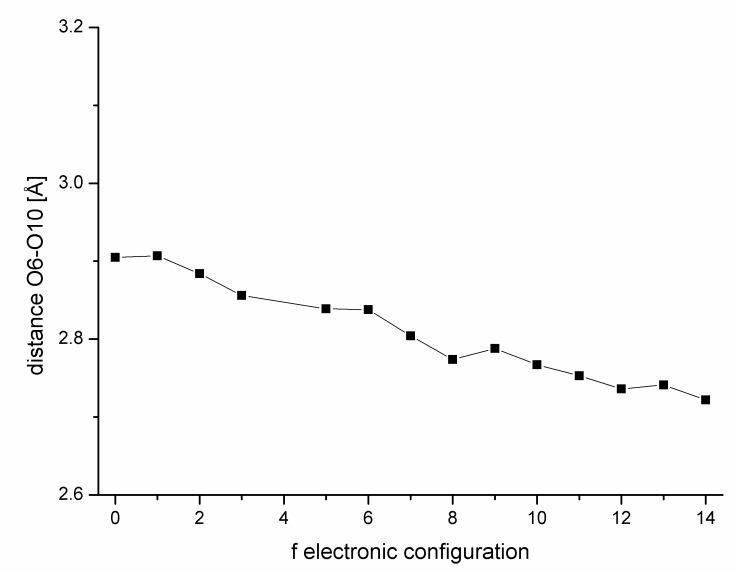

Figure S26. Distance O6-O10 vs. f electronic configuration.

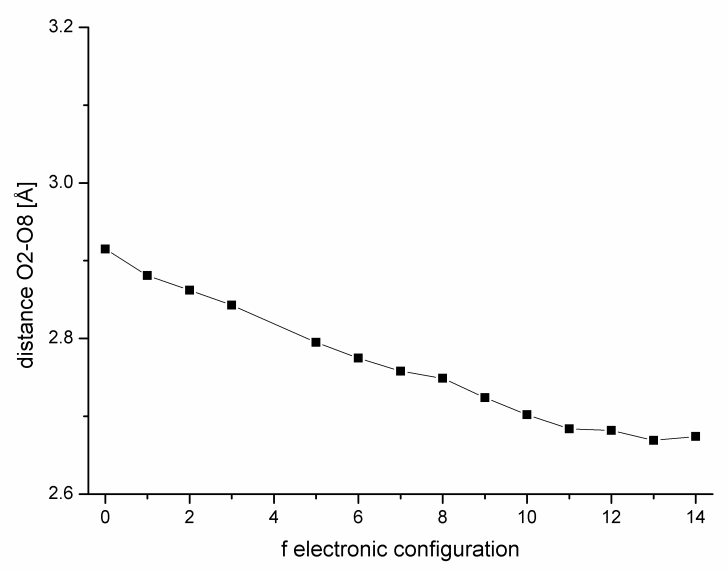

Figure S27. Distance O2-O8 vs. f electronic configuration. 


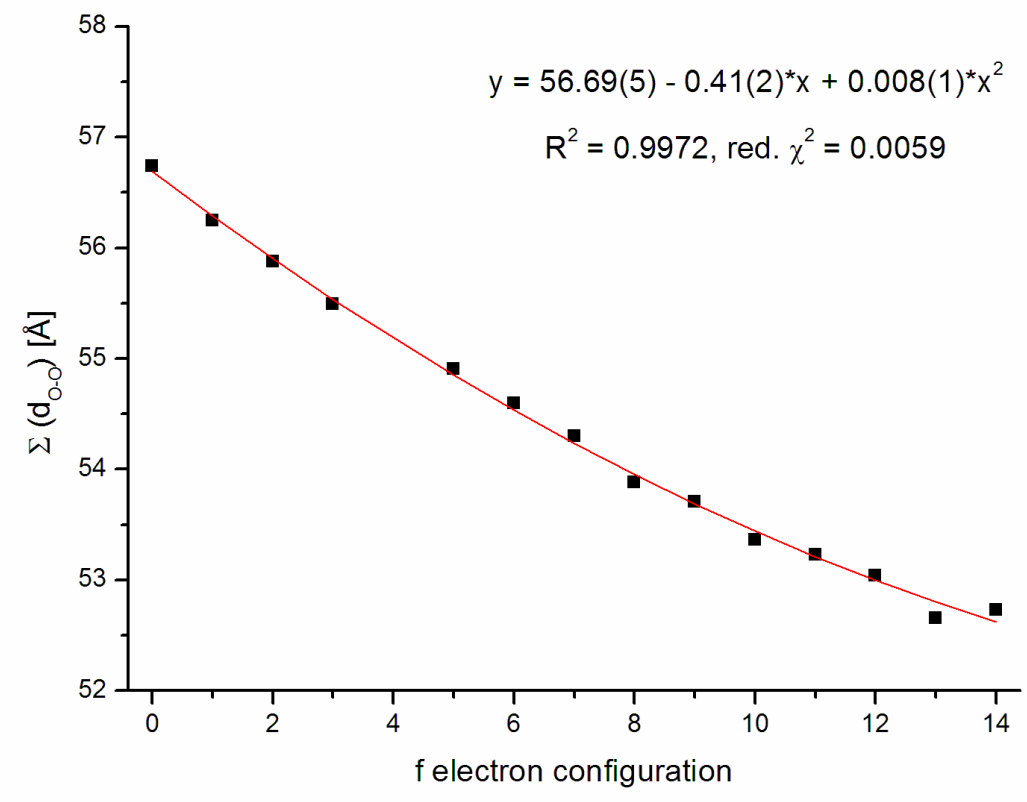

Figure S28. Sum of distance O-O vs. f electronic configuration. Quadratic fit. 


\section{TREN-SAL Lanthanide Complexes ${ }^{4-7}$}

\subsection{Structure}

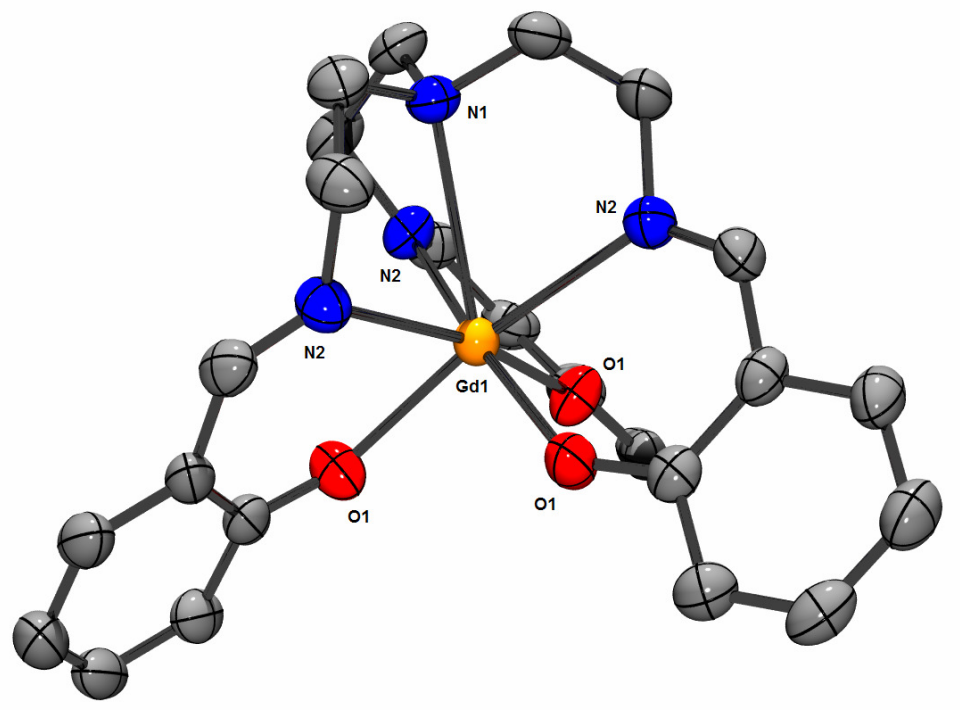

Asymmetric unit of [Gd(TREN-SAL)]. ${ }^{4}$ Thermal ellipsoid plot (Ortep 3 for Windows, ${ }^{1}$ $50 \%$ probability level) with atom numbering scheme. Hydrogens omitted for clarity.

\subsection{Bond Lengths}

Table S4. Bond lengths in TREN-SAL lanthanide complexes. ${ }^{4-7}$

\begin{tabular}{ccccc}
\hline f electrons $(\mathrm{Ln})$ & $\mathrm{d}_{\mathrm{Ln}-\mathrm{N} 1}(\mathrm{su})[\hat{\mathrm{A}}](1 \mathrm{X})$ & $\mathrm{d}_{\mathrm{Ln}-\mathrm{N} 2}(\mathrm{su})[\hat{\mathrm{A}}](3 \mathrm{X})$ & $\mathrm{d}_{\mathrm{Ln}-\mathrm{O} 1}(\mathrm{su})[\hat{\mathrm{A}}](3 \mathrm{X})$ & $\Sigma \mathrm{d}_{\mathrm{Ln}-\mathrm{X}}(\mathrm{su})[\hat{\mathrm{A}}]$ \\
\hline $0(\mathrm{La})$ & - & - & - & - \\
$1(\mathrm{Ce})^{4}$ & $2.834(4)$ & $2.605(3)$ & $2.288(3)$ & $17.513(22)$ \\
$2(\mathrm{Pr})^{4}$ & $2.814(6)$ & $2.577(5)$ & $2.282(5)$ & $17.391(36)$ \\
$3(\mathrm{Nd})^{4}$ & $2.796(5)$ & $2.573(4)$ & $2.273(3)$ & $17.334(26)$ \\
$4(\mathrm{Pm})$ & - & - & - & - \\
$5(\mathrm{Sm})^{5}$ & $2.773(4)$ & $2.531(3)$ & $2.247(3)$ & $17.107(22)$ \\
$6(\mathrm{Eu})^{5}$ & $2.761(9)$ & $2.535(7)$ & $2.233(6)$ & $17.065(48)$ \\
$7(\mathrm{Gd})^{4}$ & $2.746(4)$ & $2.511(3)$ & $2.232(2)$ & $16.975(19)$ \\
$8(\mathrm{~Tb})^{5}$ & $2.732(7)$ & $2.489(5)$ & $2.214(4)$ & $16.841(34)$ \\
$9(\mathrm{Dy})^{6}$ & $2.738(6)$ & $2.480(4)$ & $2.201(4)$ & $16.781(30)$ \\
$10(\mathrm{Ho})^{6}$ & $2.703(9)$ & $2.472(5)$ & $2.193(6)$ & $16.698(42)$ \\
$11(\mathrm{Er})^{7}$ & $2.716(8)$ & $2.455(6)$ & $2.188(5)$ & $16.645(41)$ \\
$12(\mathrm{Tm})^{7}$ & $2.713(8)$ & $2.449(6)$ & $2.167(5)$ & $16.561(41)$ \\
$13(\mathrm{Yb})^{4}$ & $2.694(10)$ & $2.430(7)$ & $2.160(6)$ & $16.464(49)$ \\
$14(\mathrm{Lu})^{7}$ & $2.722(8)$ & $2.426(6)$ & $2.162(5)$ & $16.486(41)$ \\
\hline $\mathrm{d}_{\mathrm{Ce}} / \mathrm{d} \mathrm{d}_{\mathrm{Lu}}$ & 1.041 & 1.074 & 1.058 & 1.062 \\
\hline
\end{tabular}




\section{$2.3 \mathrm{X}-\mathrm{X}$ Distances}

Table S5. X-X Distances in TREN-SAL lanthanide complexes. ${ }^{4-7}$

\begin{tabular}{|c|c|c|c|c|c|c|}
\hline f electrons (Ln) & $\mathrm{d}_{\mathrm{N} 1-\mathrm{N} 2}[\dot{\mathrm{A}}](3 \mathrm{x})$ & $\mathrm{d}_{\mathrm{N2}-\mathrm{N} 2^{\prime}}[\dot{\mathrm{A}}](3 \mathrm{x})$ & $\mathrm{d}_{\mathrm{N2}-\mathrm{O} 1}[\dot{\mathrm{A}}](3 \mathrm{x})$ & $\mathrm{d}_{\mathrm{N2}-01^{\prime}}[\dot{\mathrm{A}}](3 \mathrm{x})$ & $\mathrm{d}_{\mathrm{O} 1-\mathrm{O} 1^{\prime}}[\dot{\mathrm{A}}](3 \mathrm{x})$ & $\Sigma \mathrm{d}_{\mathrm{x}-\mathrm{x}}[\AA]$ \\
\hline $0(\mathrm{La})$ & - & - & - & - & - & - \\
\hline $1(\mathrm{Ce})^{4}$ & 2.909 & 4.073 & 2.833 & 3.424 & 3.481 & 50.160 \\
\hline $2(\mathrm{Pr})^{4}$ & 2.900 & 4.042 & 2.821 & 3.394 & 3.454 & 49.833 \\
\hline $3(\mathrm{Nd})^{4}$ & 2.908 & 4.055 & 2.819 & 3.374 & 3.425 & 49.743 \\
\hline $4(\mathrm{Pm})$ & - & - & - & - & - & \\
\hline $5(\mathrm{Sm})^{5}$ & 2.881 & 3.994 & 2.826 & 3.295 & 3.364 & 49.080 \\
\hline $6(\mathrm{Eu})^{5}$ & 2.874 & 3.997 & 2.843 & 3.272 & 3.335 & 48.963 \\
\hline $7(\mathrm{Gd})^{4}$ & 2.871 & 3.973 & 2.826 & 3.273 & 3.305 & 48.744 \\
\hline $8(\mathrm{~Tb})^{5}$ & 2.856 & 3.943 & 2.813 & 3.219 & 3.285 & 48.348 \\
\hline $9(D y)^{6}$ & 2.858 & 3.931 & 2.813 & 3.202 & 3.254 & 48.174 \\
\hline $10(\mathrm{Ho})^{6}$ & 2.840 & 3.923 & 2.819 & 3.187 & 3.223 & 47.976 \\
\hline $11(\mathrm{Er})^{*}$ & - & - & - & - & - & \\
\hline $12(\mathrm{Tm})^{*}$ & - & - & - & - & - & \\
\hline $13(\mathrm{Yb})^{4}$ & 2.836 & 3.873 & 2.794 & 3.102 & 3.163 & 47.304 \\
\hline $14(\mathrm{Lu})^{\star}$ & - & - & - & - & - & - \\
\hline $\mathrm{d}_{\mathrm{Ce}} / \mathrm{d}_{\mathrm{Lu}}$ & 1.026 & 1.052 & 1.014 & 1.104 & 1.101 & 1.060 \\
\hline
\end{tabular}

${ }^{*}$ Coordinates were not available for the calculation of X-X distances.

\subsection{Trends in Bond Lengths and X-X Distances vs. $f$ Electron Configuration}

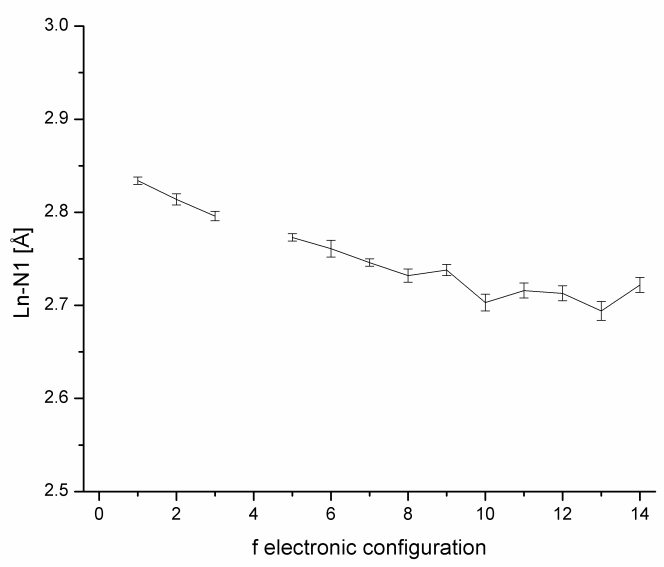

Figure S29. Bond length Ln-N1 vs. f electronic configuration. 


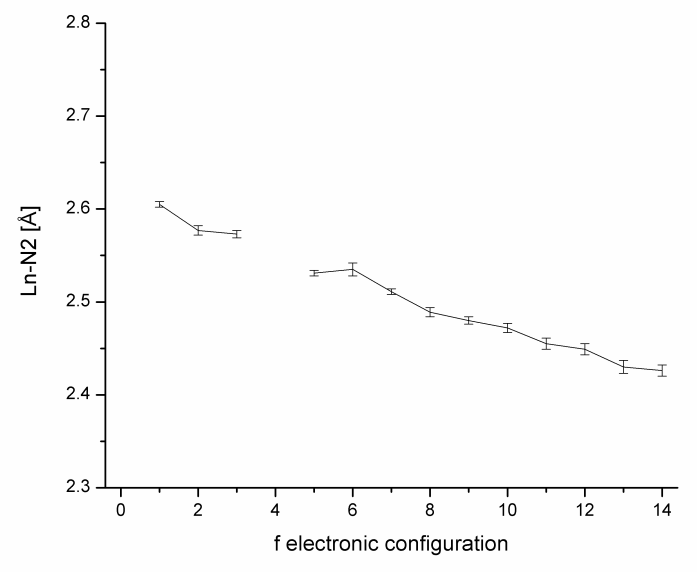

Figure S30. Bond length Ln-N2 vs. f electronic configuration.

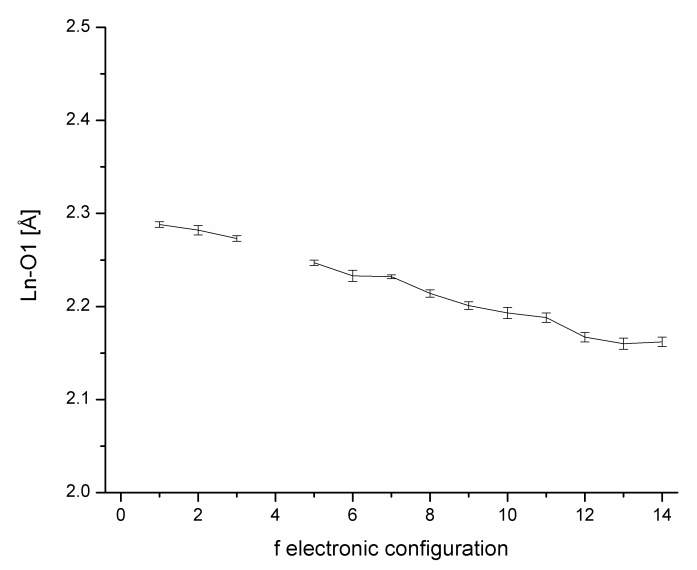

Figure S31. Bond length Ln-O1 vs. f electronic configuration.

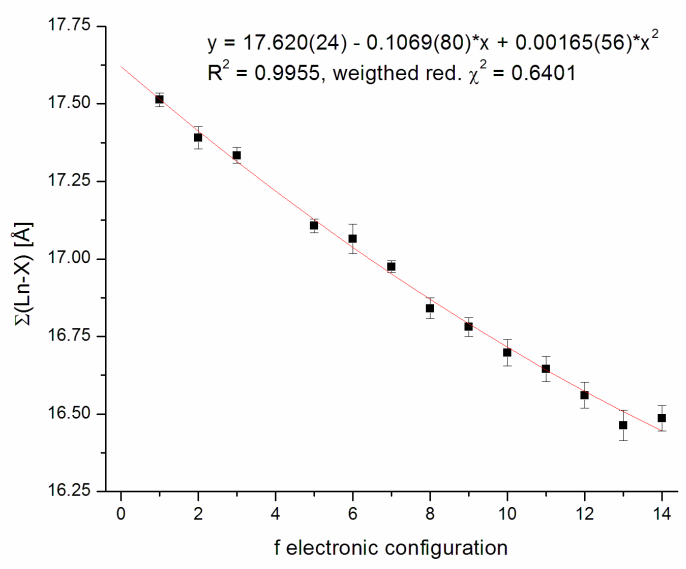

Figure S32. Sum of bond lengths $\operatorname{Ln}-X(X=N, O)$ vs. $f$ electronic configuration. Quadratic fit $\left(\chi^{2}\right.$ - weighting factor: $\left.\mathrm{su}^{-2}\right)$. 


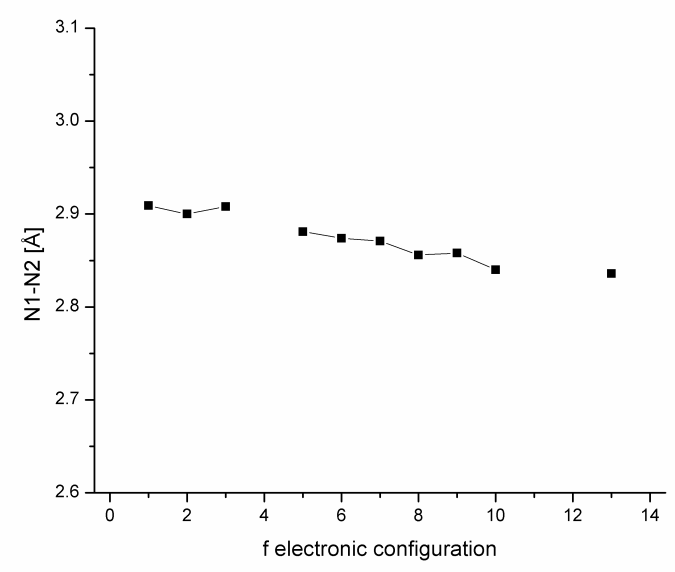

Figure S33. Distance N1-N2 vs. f electronic configuration.

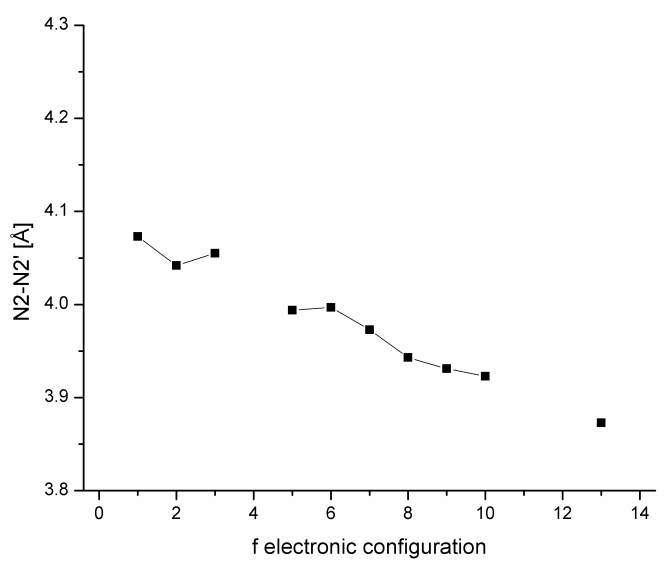

Figure S34. Distance N2-N2' vs. f electronic configuration.

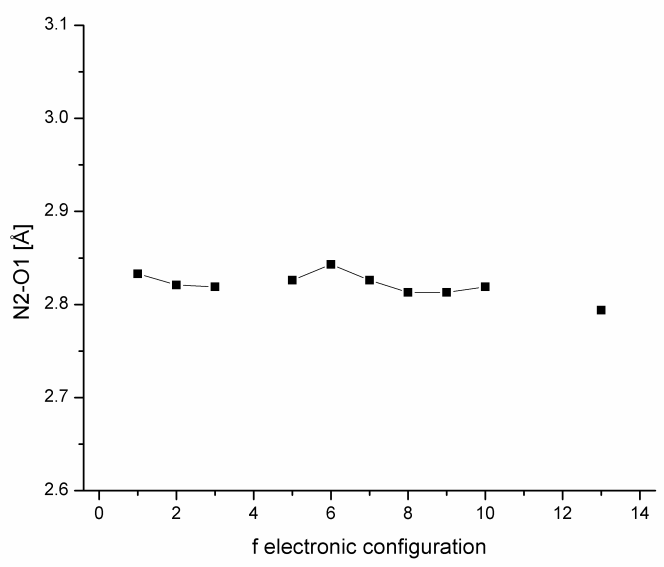

Figure S35. Distance N2-O1 vs. f electronic configuration. 


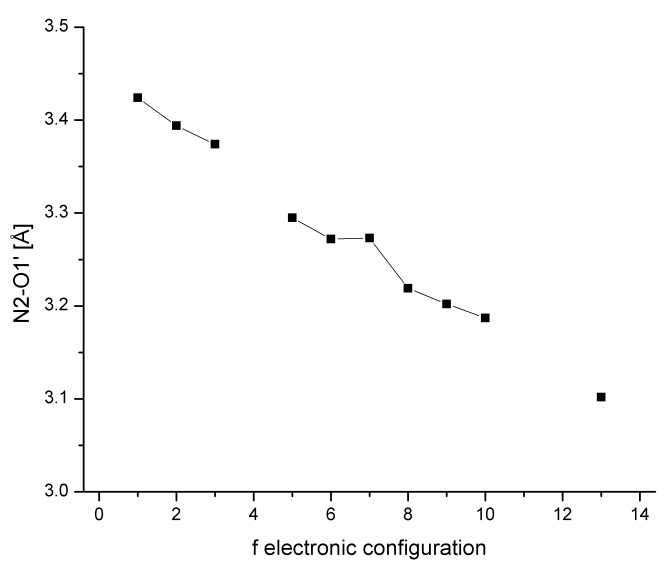

Figure S36. Distance N2-O1'vs. f electronic configuration.

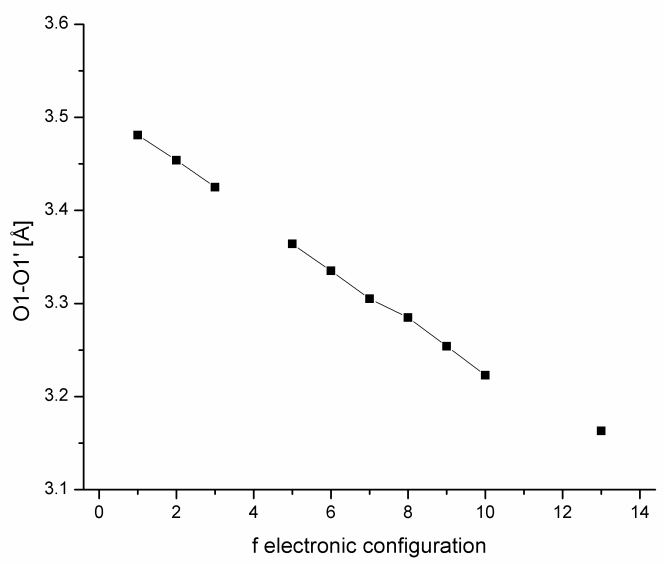

Figure S37. Distance O1-O1'vs. f electronic configuration.

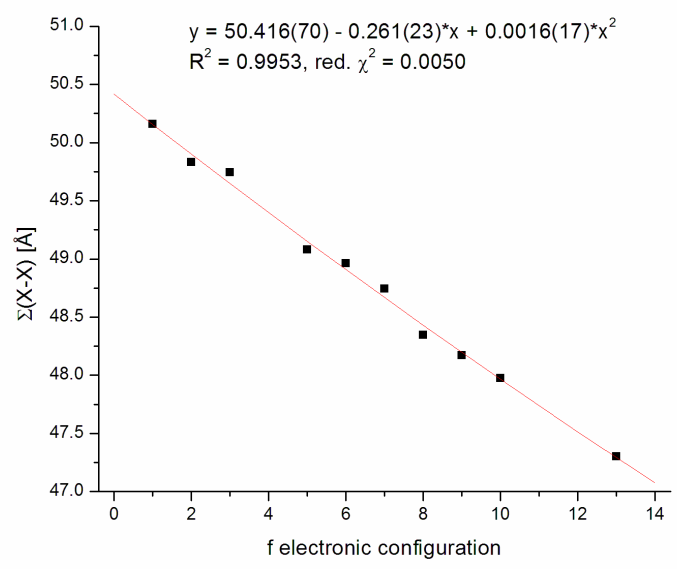

Figure S38. Sum of Distances $X-X(X=N, O$; bound to Ln) vs. $f$ electronic configuration. Quadratic fit. 


\section{3. $\left[\mathrm{Ln}(\mathrm{tptz})\left(\mathrm{NO}_{3}\right)_{3}\left(\mathrm{H}_{2} \mathrm{O}\right)\right]$ Complexes $^{8}$}

\subsection{Structure}

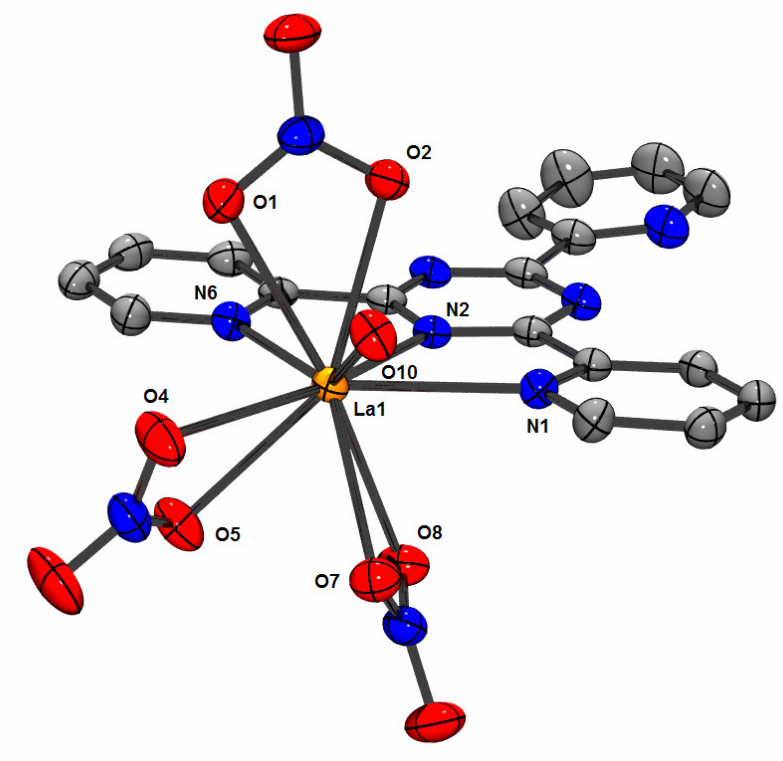

Asymmetric unit of $\left[\mathrm{La}(\mathrm{tptz})\left(\mathrm{NO}_{3}\right)_{3}\left(\mathrm{H}_{2} \mathrm{O}\right)\right]^{*} 2 \mathrm{EtOH}{ }^{8}$ Thermal ellipsoid plot (Ortep 3 for Windows, ${ }^{1} 50 \%$ probability level) with atom numbering scheme. Hydrogens and the two isolated ethanol molecules omitted for clarity.

\subsection{Bond Lengths}

Table S6. Bond lengths in $\left[\mathrm{Ln}(\mathrm{tptz})\left(\mathrm{NO}_{3}\right)_{3}\left(\mathrm{H}_{2} \mathrm{O}\right)\right]^{8}$

\begin{tabular}{|c|c|c|c|c|c|c|c|c|c|c|c|}
\hline $\begin{array}{c}\text { f electrons } \\
\text { (Ln) }\end{array}$ & $\begin{array}{c}\mathrm{d}_{\mathrm{Ln}-\mathrm{N} 1}(\mathrm{su}) \\
{[\AA \AA \AA}\end{array}$ & $\begin{array}{c}\mathrm{d}_{\mathrm{Ln}-\mathrm{N} 2}(\mathrm{su}) \\
{[\AA \AA]}\end{array}$ & $\begin{array}{c}\mathrm{d}_{\mathrm{Ln}-\mathrm{N} 6}(\mathrm{su}) \\
{[\AA \AA A]}\end{array}$ & $\begin{array}{c}\mathrm{d}_{\mathrm{Ln}-\mathrm{O} 1}(\mathrm{su}) \\
{[\AA \AA} \\
{[\mathrm{A}]}\end{array}$ & $\begin{array}{c}\mathrm{d}_{\mathrm{Ln}-\mathrm{O} 2}(\mathrm{su}) \\
{[\AA \AA \AA}\end{array}$ & $\begin{array}{c}\mathrm{d}_{\mathrm{Ln}-04}(\mathrm{su}) \\
{[\AA \AA \AA}\end{array}$ & $\begin{array}{c}\mathrm{d}_{\mathrm{Ln}-05}(\mathrm{su}) \\
{[\AA \AA]}\end{array}$ & $\begin{array}{c}\mathrm{d}_{\mathrm{Ln}-\mathrm{O} 7}(\mathrm{su}) \\
{[\AA \AA \AA} \\
\end{array}$ & $\begin{array}{c}\mathrm{d}_{\text {Ln-08 }}(\mathrm{su}) \\
{[\AA \AA \AA}\end{array}$ & $\begin{array}{c}\mathrm{d}_{\mathrm{Ln-010}}(\mathrm{su}) \\
{[\AA \AA \AA}\end{array}$ & $\begin{array}{c}\sum \mathrm{d}_{\mathrm{Ln}-\mathrm{X}}(\mathrm{su}) \\
{[\AA \AA}\end{array}$ \\
\hline 0 (La) & $2.667(2)$ & $2.691(1)$ & $2.703(1)$ & $2.617(1)$ & $2.570(1)$ & $2.588(2)$ & $2.631(1)$ & $2.595(1)$ & $2.631(1)$ & $2.452(1)$ & $26.145(12)$ \\
\hline $1(\mathrm{Ce})$ & $2.660(4)$ & $2.678(4)$ & $2.696(4)$ & $2.598(4)$ & $2.549(4)$ & $2.565(4)$ & $2.618(4)$ & $2.585(4)$ & $2.611(4)$ & $2.424(4)$ & $25.984(40)$ \\
\hline $2(\operatorname{Pr})$ & $2.630(3)$ & $2.644(3)$ & $2.668(3)$ & $2.583(3)$ & $2.524(3)$ & $2.546(3)$ & $2.594(3)$ & $2.556(2)$ & $2.592(3)$ & $2.412(3)$ & $25.749(29)$ \\
\hline $3(\mathrm{Nd})$ & 2.614(3) & $2.629(3)$ & $2.658(3)$ & $2.568(3)$ & $2.506(3)$ & $2.534(3)$ & $2.584(3)$ & $2.541(3)$ & $2.584(3)$ & $2.400(3)$ & $25.618(30)$ \\
\hline $4(\mathrm{Pm})$ & - & - & - & - & - & - & - & - & - & - & - \\
\hline $5(\mathrm{Sm})$ & $2.593(2)$ & $2.603(2)$ & $2.629(2)$ & $2.546(2)$ & $2.481(2)$ & $2.505(2)$ & $2.566(2)$ & $2.511(2)$ & $2.557(2)$ & $2.368(2)$ & $25.359(20)$ \\
\hline $6(\mathrm{Eu})$ & $2.576(2)$ & $2.579(2)$ & $2.619(2)$ & $2.536(2)$ & $2.465(2)$ & $2.490(2)$ & $2.558(2)$ & $2.496(2)$ & $2.544(2)$ & $2.349(2)$ & $25.212(20)$ \\
\hline $7(\mathrm{Gd})$ & $2.569(2)^{\star}$ & $2.567(2)^{\star}$ & $2.607(2)$ & $2.532(2)$ & $2.456(2)$ & $2.485(2)^{*}$ & $2.555(2)$ & $2.481(2)^{\star}$ & $2.538(2)$ & $2.337(2)$ & $25.127(20)$ \\
\hline $8(\mathrm{~Tb})$ & $2.555(2)^{*}$ & $2.545(2)^{*}$ & $2.592(2)$ & $2.523(2)^{*}$ & $2.439(2)$ & $2.464(2)$ & $2.552(2)$ & $2.467(2)$ & $2.520(2)^{\star}$ & $2.322(2)$ & $24.979(20)$ \\
\hline 9 (Dy) & $2.547(2)^{*}$ & $2.534(2)^{\star}$ & $2.582(2)$ & $2.516(2)^{\star}$ & $2.426(2)$ & $2.451(2)$ & $2.545(2)$ & $2.453(2)$ & $2.515(2)^{\star}$ & $2.311(2)$ & $24.880(20)$ \\
\hline $10(\mathrm{Ho})$ & $2.537(2)^{\star}$ & $2.524(2)^{\star}$ & $2.573(2)$ & $2.512(2)^{*}$ & $2.416(1)$ & $2.442(2)$ & $2.544(2)$ & $2.442(1)$ & $2.506(2)^{\star}$ & $2.303(2)$ & $24.799(18)$ \\
\hline $11(\mathrm{Er})$ & $2.528(2)^{\star}$ & $2.513(2)^{\star}$ & $2.562(3)$ & $2.503(3)^{*}$ & $2.401(3)$ & $2.429(2)$ & $2.544(3)$ & $2.432(2)$ & $2.495(2)^{\star}$ & $2.292(3)$ & $24.699(25)$ \\
\hline $12(\mathrm{Tm})$ & $2.508(4)$ & $2.508(4)$ & $2.519(3)$ & $2.542(3)^{*}$ & $2.404(3)$ & $2.419(3)$ & $2.520(3)^{\star}$ & $2.437(3)$ & $2.528(3)$ & $2.262(3)$ & $24.647(32)$ \\
\hline $13(\mathrm{Yb})$ & $2.510(2)^{*}$ & $2.490(2)^{\star}$ & $2.541(2)$ & $2.504(2)^{\star}$ & $2.377(2)$ & $2.399(2)$ & $2.567(2)$ & $2.413(2)$ & $2.482(2)^{\star}$ & $2.270(2)$ & $24.553(20)$ \\
\hline $14(\mathrm{Lu})$ & - & - & - & - & - & - & - & - & - & - & - \\
\hline $\mathrm{d}_{\mathrm{La}} / \mathrm{d}_{\mathrm{Yb}}$ & 1.063 & 1.081 & 1.064 & 1.080 & 1.081 & 1.079 & 1.075 & 1.045 & 1.060 & 1.025 & 1.065 \\
\hline
\end{tabular}




\subsection{X-X Distances}

Table S7. Distances $X-X(X=N, O$ bound to $\mathrm{Ln})$ in $\left[\mathrm{Ln}(\mathrm{tptz})\left(\mathrm{NO}_{3}\right)_{3}\left(\mathrm{H}_{2} \mathrm{O}\right)\right]^{8}$

\begin{tabular}{|c|c|c|c|c|c|c|c|c|c|c|c|c|c|c|c|c|c|c|c|c|c|c|}
\hline $\begin{array}{l}\text { electrons } \\
\text { (Ln) }\end{array}$ & $\begin{array}{c}\mathrm{d}_{\mathrm{N1}-\mathrm{N} 2} \\
{[\AA \AA}\end{array}$ & $\begin{array}{c}\mathrm{d}_{\mathrm{N} 1-02} \\
{[\hat{A}]}\end{array}$ & $\begin{array}{c}\mathrm{d}_{\mathrm{N} 1-010} \\
{[\AA]}\end{array}$ & $\begin{array}{c}\mathrm{d}_{\mathrm{N} 1-07} \\
{[\AA]}\end{array}$ & $\begin{array}{c}\mathrm{d}_{\mathrm{N1}-08} \\
{[\AA \AA \AA}\end{array}$ & 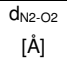 & $\begin{array}{c}\mathrm{d}_{\mathrm{O2-010}} \\
[\AA]]\end{array}$ & $\begin{array}{c}\mathrm{d}_{\mathrm{O} 10-07} \\
{[\AA \AA]}\end{array}$ & $\begin{array}{c}\mathrm{d}_{07-08} \\
{[\AA \hat{A}]}\end{array}$ & $\begin{array}{c}\mathrm{d}_{\mathrm{OB-N} 2} \\
{[\AA \AA \AA}\end{array}$ & $\begin{array}{c}\mathrm{d}_{\mathrm{N} 6-\mathrm{O} 1} \\
{[\AA \AA}\end{array}$ & $\begin{array}{c}d_{01-04} \\
[\AA]]\end{array}$ & $\begin{array}{c}\mathrm{d}_{\mathrm{O} 4 \mathrm{-05}} \\
{[\AA \AA]}\end{array}$ & $\begin{array}{c}\mathrm{d}_{\mathrm{O5-N6}} \\
{[\AA]}\end{array}$ & $\begin{array}{c}\mathrm{d}_{\mathrm{N} 2-\mathrm{N} 6} \\
{[\AA \AA]}\end{array}$ & 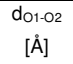 & 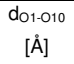 & $\begin{array}{c}\mathrm{d}_{010-04} \\
[\AA]]\end{array}$ & $\begin{array}{c}\mathrm{d}_{04-07} \\
{[\AA \AA]}\end{array}$ & $\begin{array}{c}\mathrm{d}_{07-05} \\
[\AA]]\end{array}$ & $\begin{array}{c}d_{05-08} \\
{[\AA \AA]}\end{array}$ & $\begin{array}{c}\Sigma \mathrm{d}_{\mathrm{x}-\mathrm{x}} \\
{[\AA \AA \AA]}\end{array}$ \\
\hline 0 (La) & 2.705 & 3.175 & 3.195 & 3.246 & 2.979 & 2.918 & 3.025 & 3.115 & 2.164 & 3.145 & 3.048 & 3.042 & 2.152 & 3.089 & 2.698 & 2.159 & 3.080 & 3.042 & 3.154 & 3.028 & 2.993 & 61.152 \\
\hline $1(\mathrm{Ce})$ & 2.715 & 3.157 & 3.176 & 3.237 & 2.967 & 2.900 & 3.002 & 3.084 & 2.165 & 3.124 & 3.045 & 3.014 & 2.161 & 3.078 & 2.700 & 2.162 & 3.039 & 2.974 & 3.100 & 2.981 & 2.949 & 60.730 \\
\hline $2(\mathrm{Pr})$ & 2.701 & 3.141 & 3.148 & 3.179 & 2.934 & 2.871 & 2.975 & 3.060 & 2.163 & 3.080 & 3.004 & 2.974 & 2.159 & 3.041 & 2.691 & 2.161 & 3.014 & 2.959 & 3.080 & 2.950 & 2.906 & 60.191 \\
\hline $3(\mathrm{Nd})$ & 2.702 & 3.121 & 3.128 & 3.149 & 2.918 & 2.848 & 2.971 & 3.032 & 2.170 & 3.053 & 2.994 & 2.955 & 2.162 & 3.026 & 2.687 & 2.160 & 2.993 & 2.928 & 3.057 & 2.931 & 2.889 & 59.874 \\
\hline $4(\mathrm{Pm})$ & - & - & - & - & - & - & - & - & - & - & - & - & - & - & - & - & - & - & - & - & - & - \\
\hline $5(\mathrm{Sm})$ & 2.703 & 3.099 & 3.087 & 3.111 & 2.902 & 2.833 & 2.928 & 3.004 & 2.168 & 3.001 & 2.945 & 2.906 & 2.155 & 2.990 & 2.677 & 2.163 & 2.958 & 2.890 & 3.017 & 2.887 & 2.842 & 59.266 \\
\hline $6(\mathrm{Eu})$ & 2.691 & 3.089 & 3.066 & 3.083 & 2.884 & 2.816 & 2.914 & 2.974 & 2.171 & 2.968 & 2.929 & 2.886 & 2.153 & 2.964 & 2.676 & 2.150 & 2.942 & 2.869 & 3.003 & 2.876 & 2.820 & 3.924 \\
\hline $7(\mathrm{Gd})$ & 2.692 & 3.077 & 3.056 & 3.068 & 2.874 & 2.808 & 2.895 & 2.963 & 2.163 & 2.955 & 2.914 & 2.869 & 2.157 & 2.953 & 2.667 & 2.159 & 2.925 & 2.862 & 2.993 & 2.859 & 2.806 & 58.715 \\
\hline $8(\mathrm{~Tb})$ & 2.688 & 3.065 & 3.031 & 3.043 & 2.859 & 2.789 & 2.881 & 2.945 & 2.158 & 2.920 & 2.901 & 2.846 & 2.152 & 2.936 & 2.659 & 2.153 & 2.911 & 2.838 & 2.972 & 2.840 & 2.788 & 58.375 \\
\hline 9 (Dy) & 2.690 & 3.052 & 3.019 & 3.025 & 2.850 & 2.775 & 2.868 & 2.926 & 2.162 & 2.906 & 2.881 & 2.825 & 2.149 & 2.925 & 2.656 & 2.156 & 2.900 & 2.824 & 2.958 & 2.822 & 2.770 & 58.139 \\
\hline $10(\mathrm{Ho})$ & 2.686 & 3.036 & 3.004 & 3.009 & 2.842 & 2.763 & 2.859 & 2.911 & 2.159 & 2.889 & 2.871 & 2.816 & 2.151 & 2.909 & 2.659 & 2.156 & 2.890 & 2.818 & 2.947 & 2.813 & 2.754 & 57.942 \\
\hline $11(\mathrm{Er})$ & 2.686 & 3.026 & 2.988 & 2.993 & 2.827 & 2.748 & 2.834 & 2.906 & 2.156 & 2.873 & 2.855 & 2.800 & 2.144 & 2.899 & 2.656 & 2.156 & 2.871 & 2.814 & 2.930 & 2.801 & 2.744 & 57.707 \\
\hline $12(\mathrm{Tm})$ & 2.647 & 3.056 & 3.015 & 2.980 & 2.797 & 2.767 & 2.836 & 2.913 & 2.167 & 2.860 & 2.853 & 2.803 & 2.159 & 2.899 & 2.631 & 2.151 & 2.851 & 2.748 & 2.938 & 2.753 & 2.731 & 57.555 \\
\hline $13(\mathrm{Yb})$ & 2.677 & 3.007 & 2.974 & 2.977 & 2.826 & 2.729 & 2.825 & 2.876 & 2.162 & 2.836 & 2.842 & 2.776 & 2.141 & 2.888 & 2.642 & 2.148 & 2.861 & 2.788 & 2.906 & 2.785 & 2.719 & 57.385 \\
\hline $14(\mathrm{Lu})$ & - & - & - & - & - & - & - & - & - & - & - & - & - & - & - & - & - & - & - & - & - & - \\
\hline$d_{q_{a}} / d_{Y b}$ & 1.010 & 1.056 & 1.074 & 1.080 & 1.054 & 1.069 & 1.071 & 1.083 & 1.001 & 1.109 & 1.072 & 1.096 & 1.005 & 1.070 & 1.021 & 1.005 & 1.077 & 1.091 & 1.085 & 1.087 & 1.101 & 1.066 \\
\hline
\end{tabular}

\subsection{Trends in Bond Lengths and X-X Distances vs. $f$ Electron Configuration} Bond Lengths:

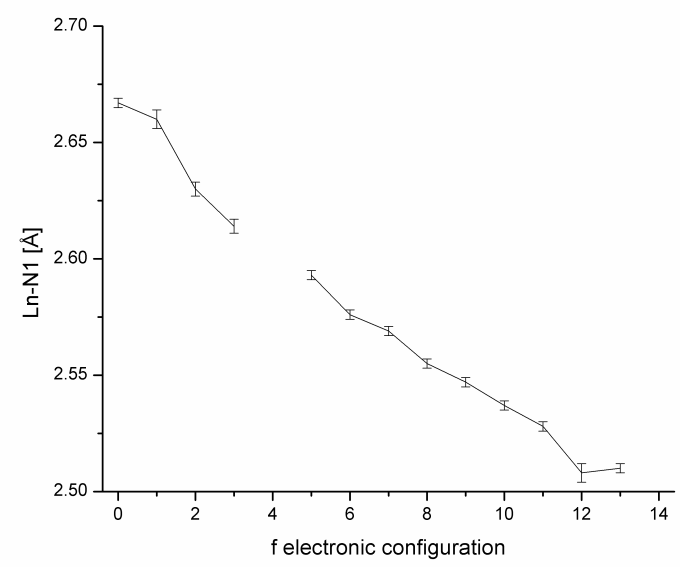

Figure S39. Bond length Ln-N1 vs. f electronic configuration. 


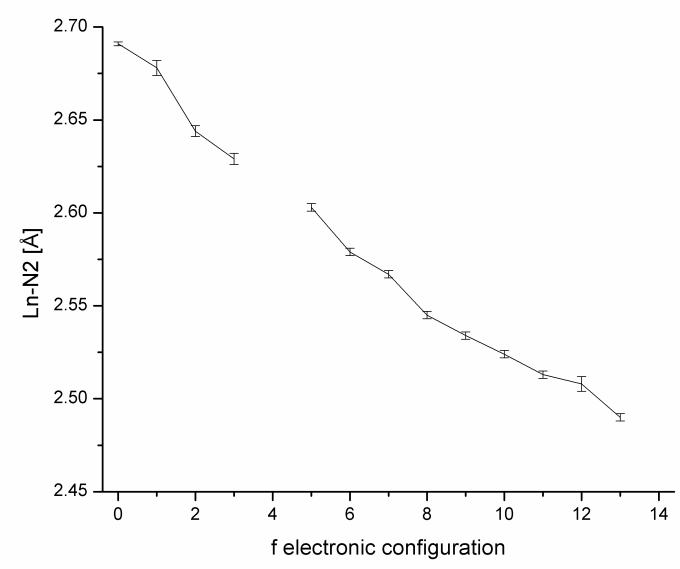

Figure S40. Bond length Ln-N2 vs. f electronic configuration.

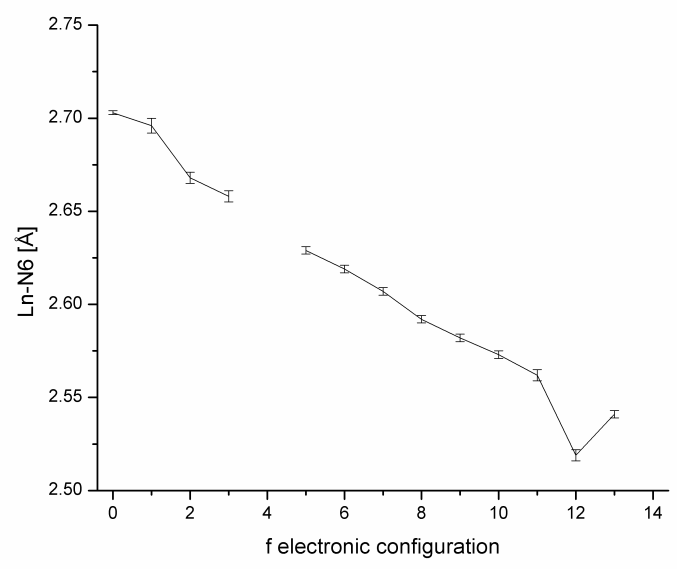

Figure S41. Bond length Ln-N6 vs. f electronic configuration.

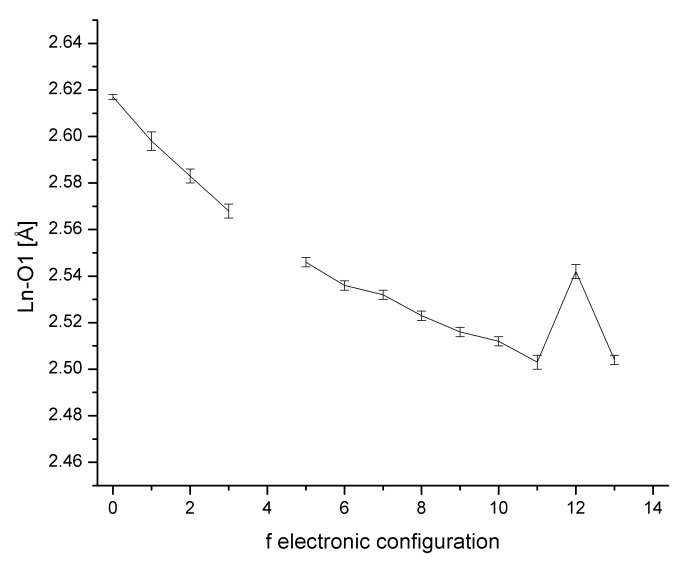

Figure S42. Bond length Ln-O1 vs. f electronic configuration. 


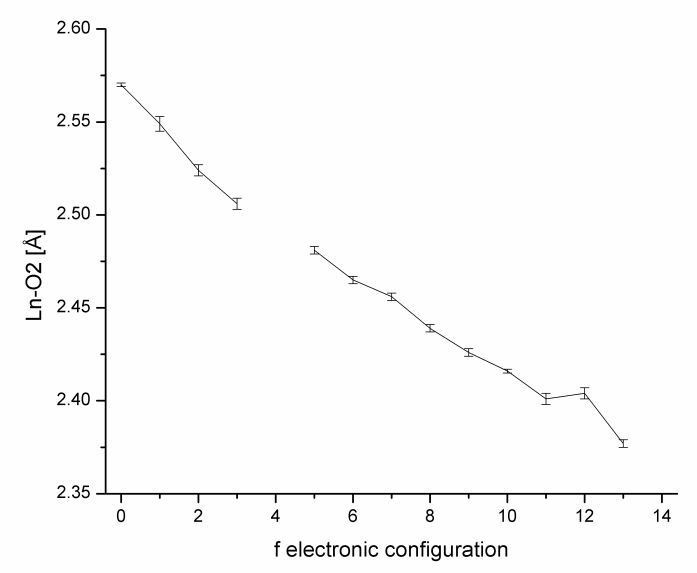

Figure S43. Bond length Ln-O2 vs. f electronic configuration.

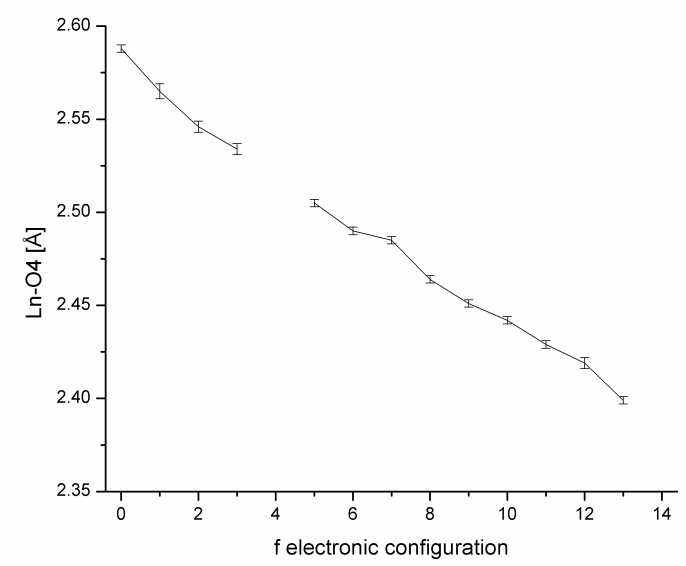

Figure S44. Bond length Ln-O4 vs. f electronic configuration.

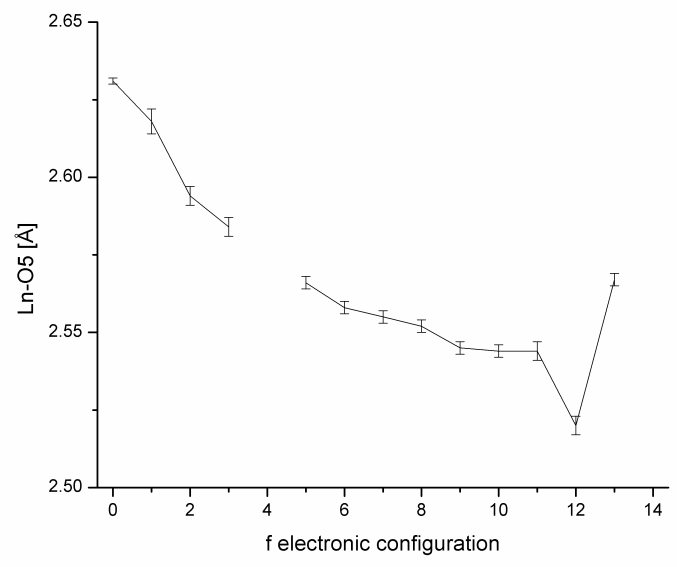

Figure S45. Bond length Ln-O5 vs. f electronic configuration. 


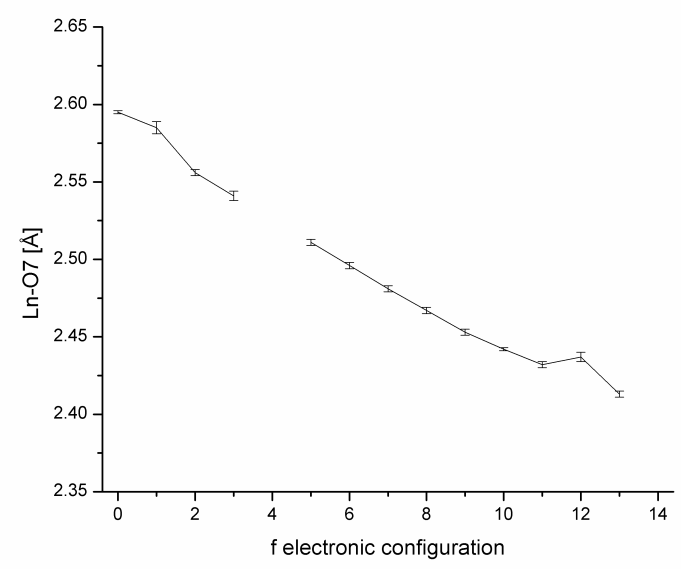

Figure S46. Bond length Ln-O7 vs. f electronic configuration.

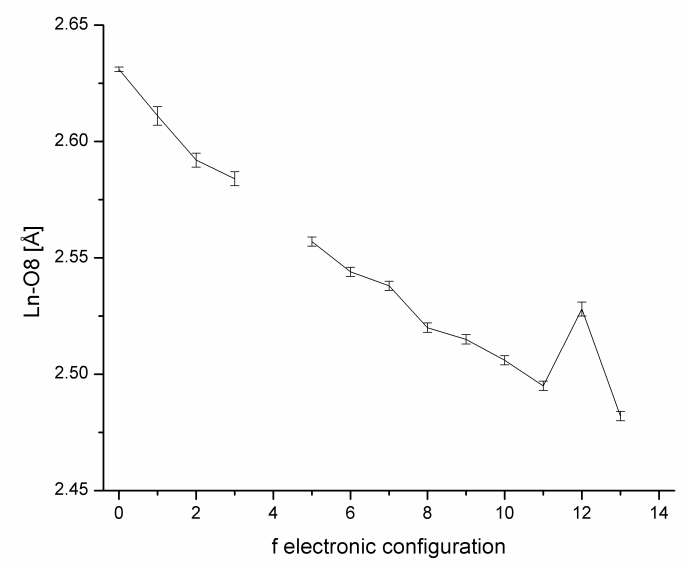

Figure S47. Bond length Ln-O8 vs. f electronic configuration.

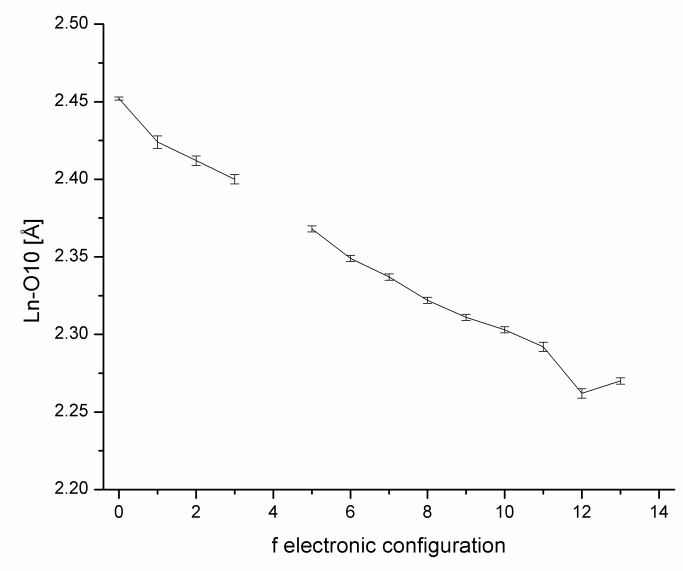

Figure S48. Bond length Ln-O10 vs. f electronic configuration. 


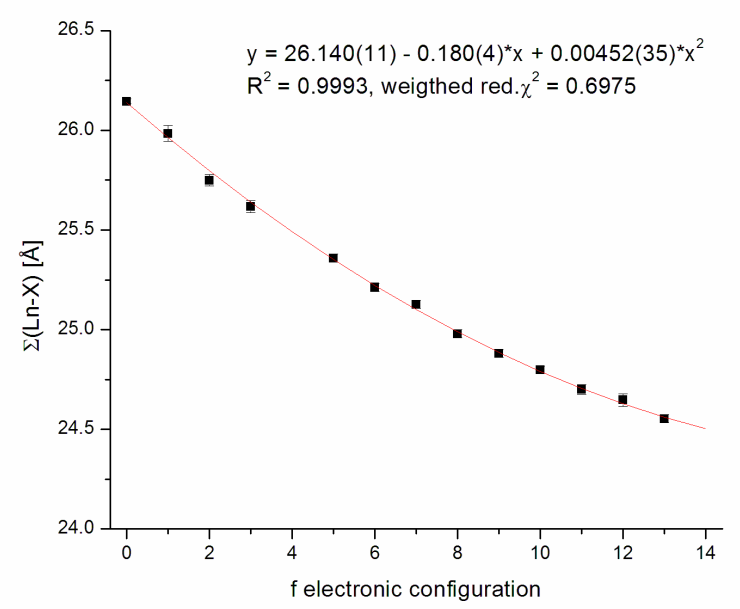

Figure S49. Sum of bond lengths $\operatorname{Ln}-X \operatorname{Ln}-X(X=N, O)$ vs. $f$ electronic configuration. Quadratic fit $\left(\chi^{2}\right.$ - weighting factor: $\left.\mathrm{su}^{-2}\right)$.

Distances $X-X(X=N, O)$ :

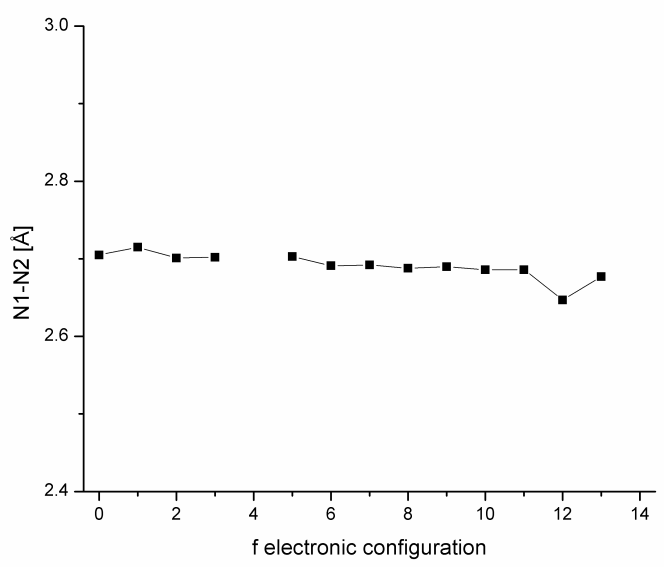

Figure S50. Distance N1-N2 vs. f electronic configuration.

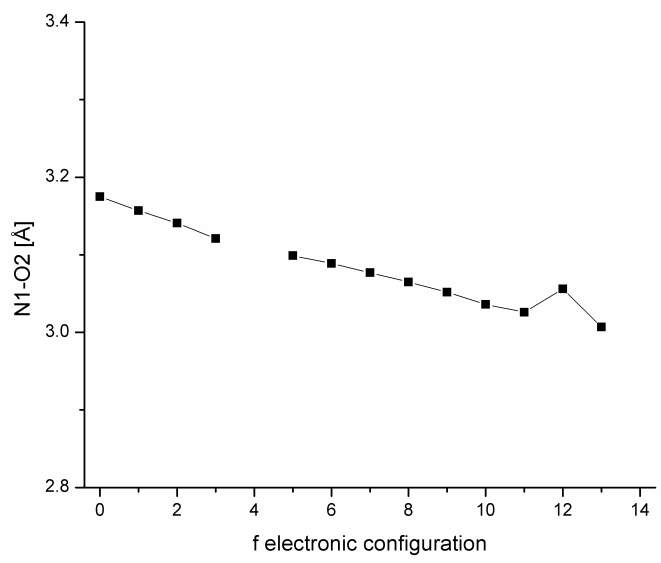

Figure S51. Distance N1-O2 vs. f electronic configuration. 


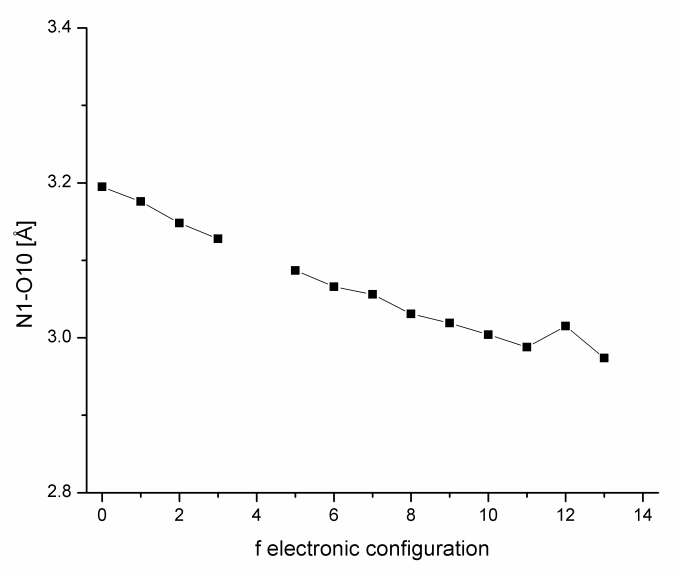

Figure S52. Distance N1-O10 vs. f electronic configuration.

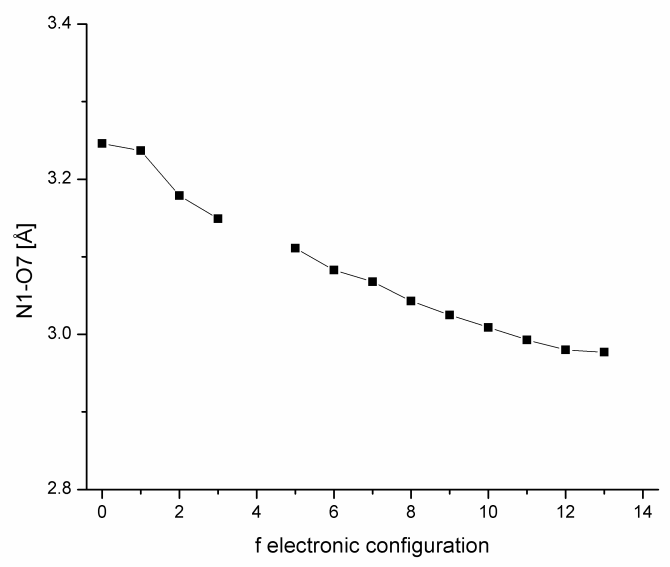

Figure S53. Distance N1-O7 vs. f electronic configuration.

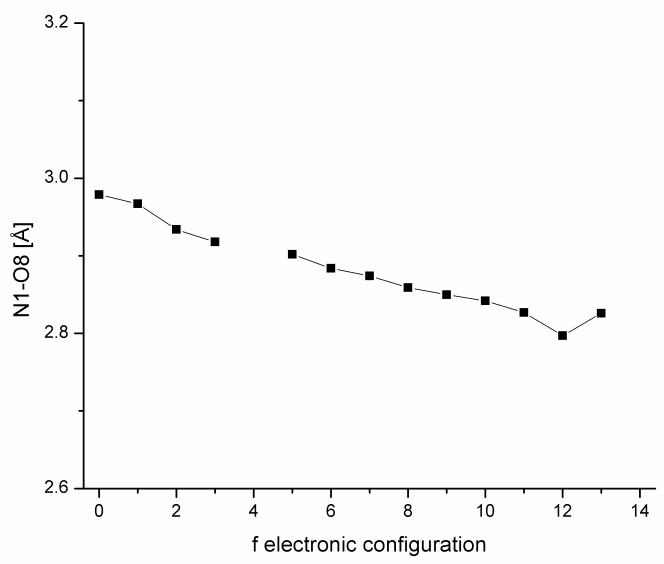

Figure S54. Distance N1-O8 vs. f electronic configuration. 


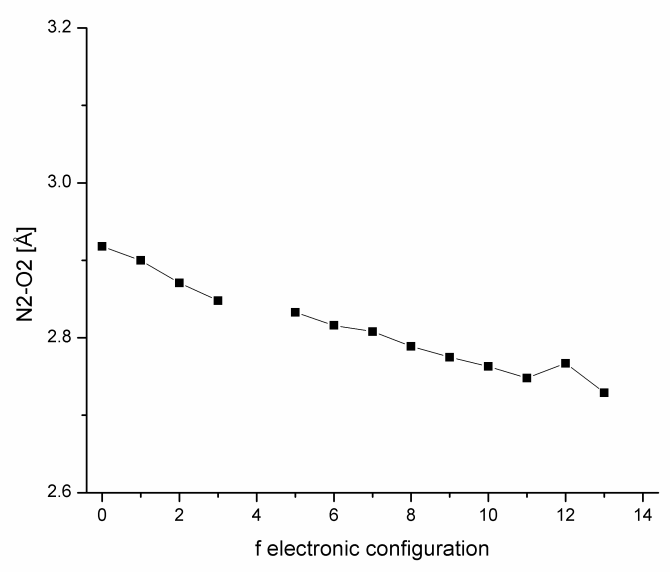

Figure S55. Distance N2-O2 vs. f electronic configuration.

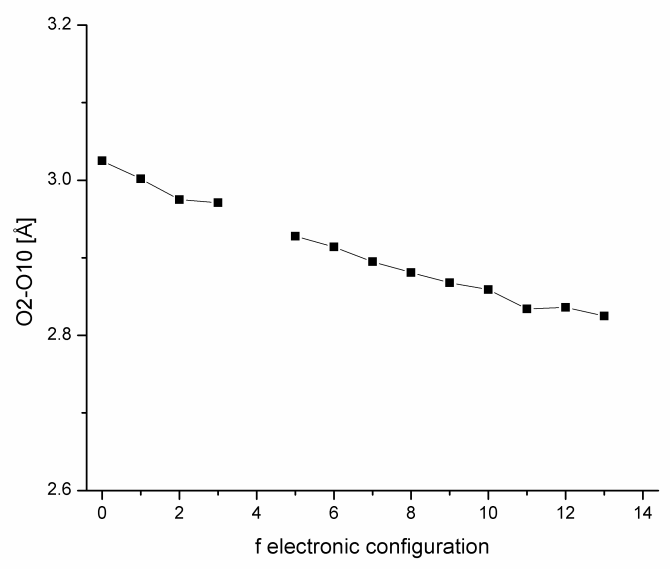

Figure S56. Distance O2-O10 vs. f electronic configuration.

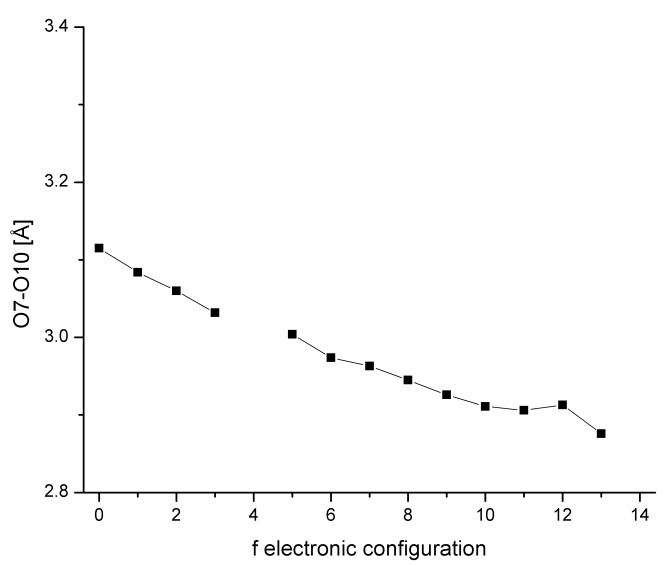

Figure S57. Distance O7-O10 vs. f electronic configuration. 


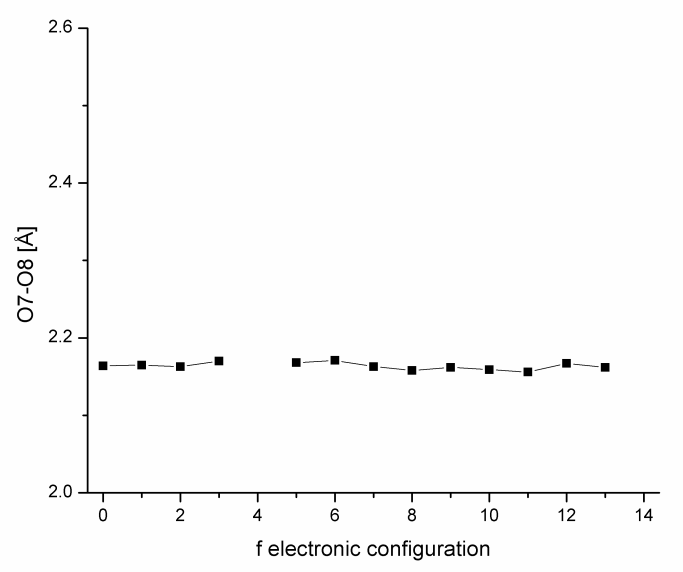

Figure S58. Distance O7-O8 vs. f electronic configuration.

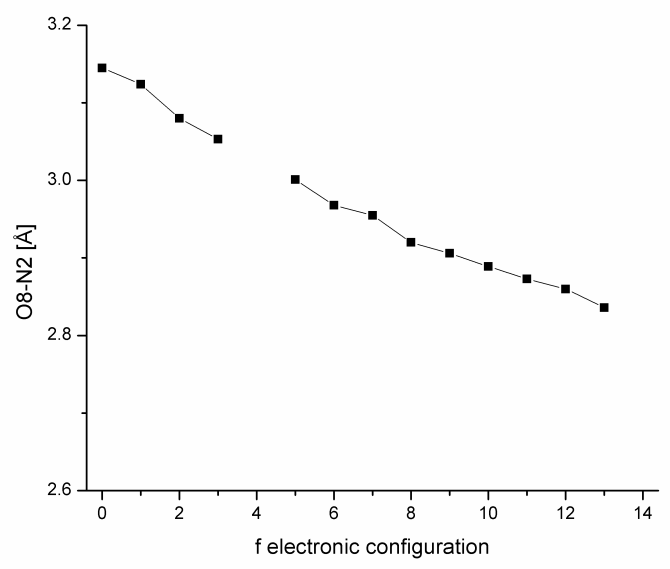

Figure S59. Distance O8-N2 vs. f electronic configuration.

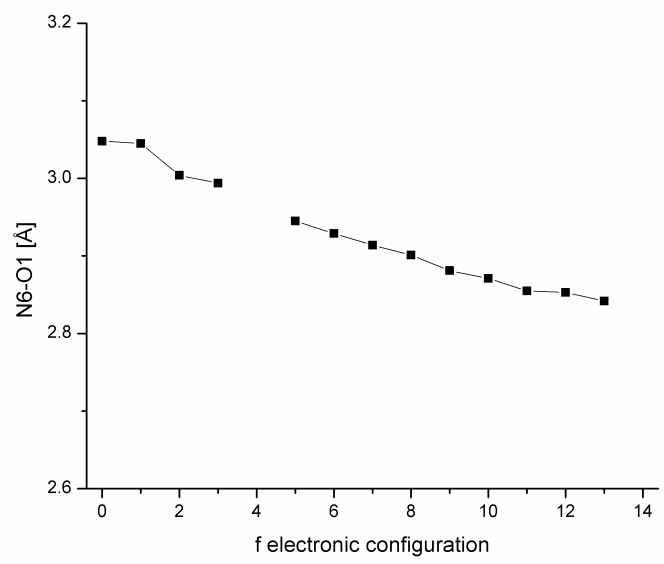

Figure S60. Distance N6-O1 vs. f electronic configuration. 


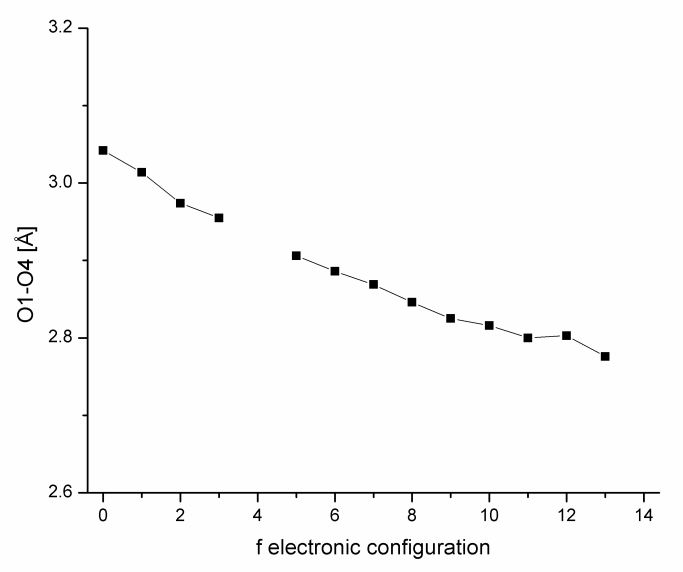

Figure S61. Distance O1-O4 vs. f electronic configuration.

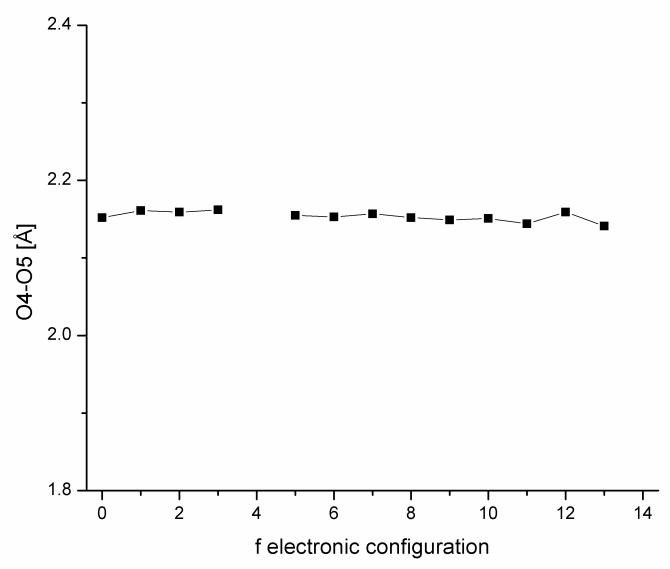

Figure S62. Distance O4-O5 vs. f electronic configuration.

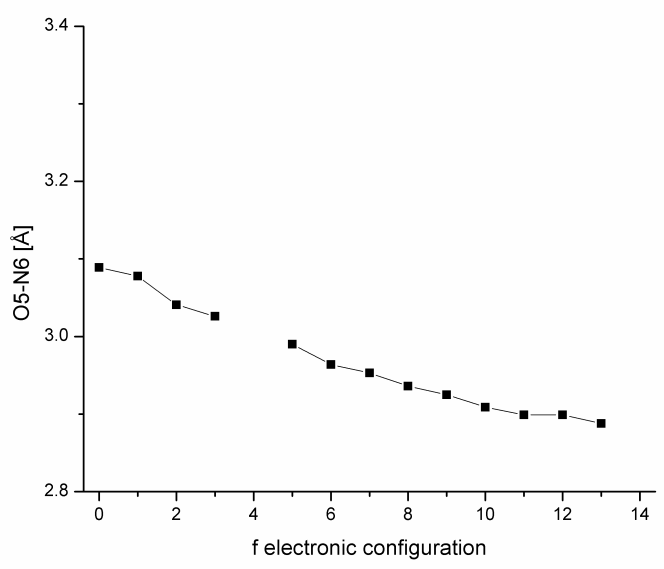

Figure S63. Distance O5-N6 vs. f electronic configuration. 


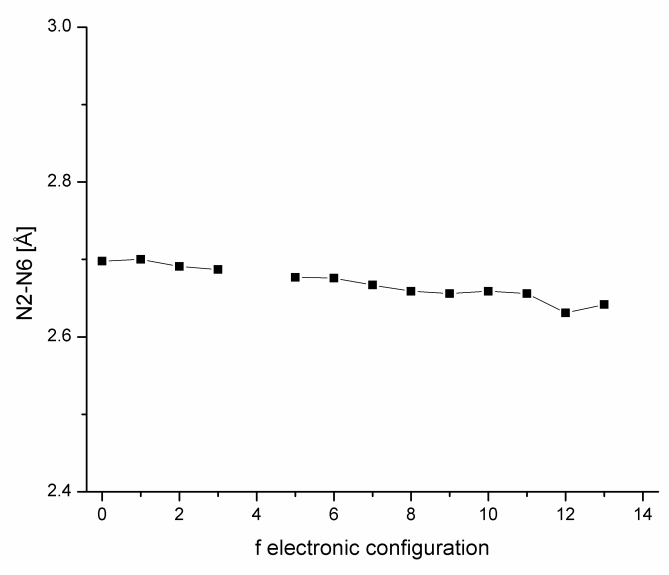

Figure S64. Distance N2-N6 vs. f electronic configuration.

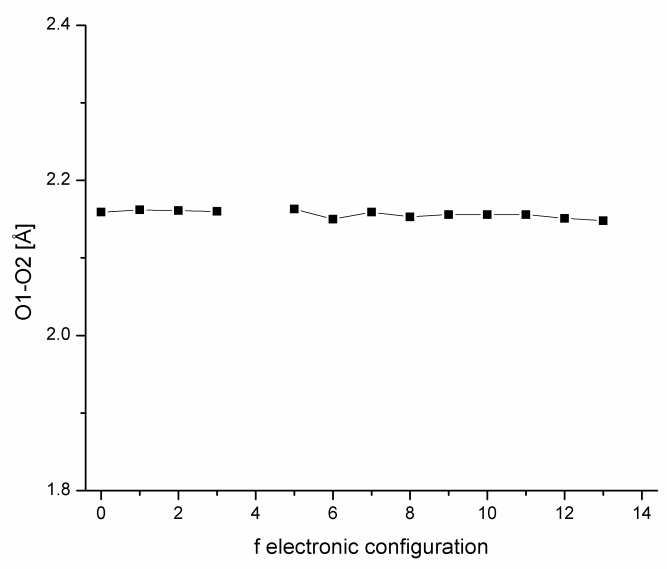

Figure S65. Distance O1-O2 vs. f electronic configuration.

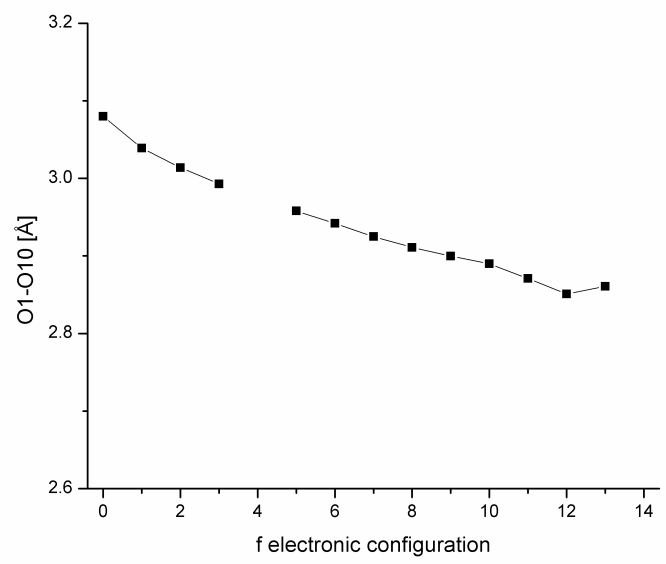

Figure S66. Distance O1-O10 vs. f electronic configuration. 


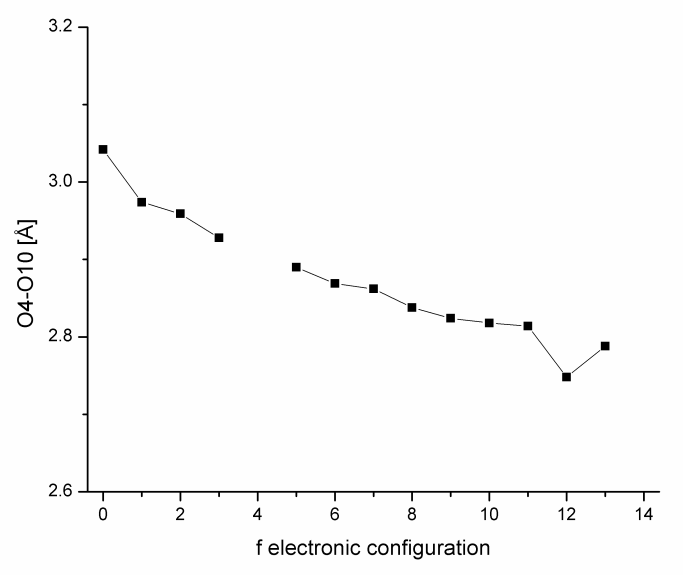

Figure S67. Distance O4-O10 vs. f electronic configuration.

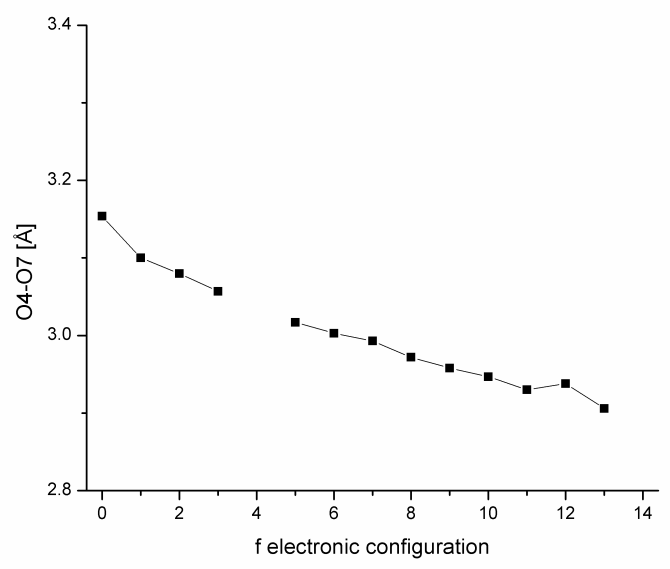

Figure S68. Distance O4-O7 vs. f electronic configuration.

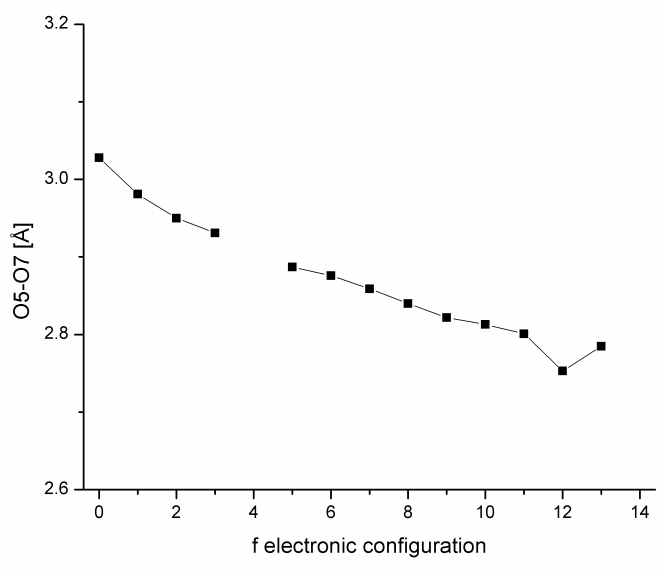

Figure S69. Distance O5-O7 vs. f electronic configuration. 


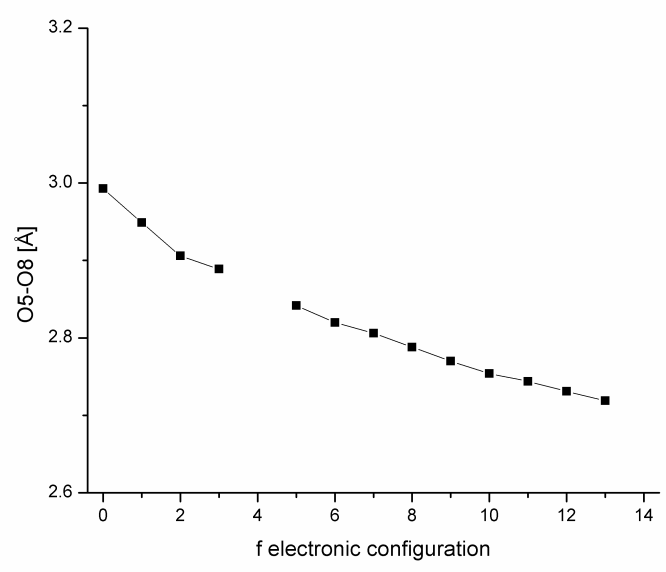

Figure S70. Distance O5-O8 vs. f electronic configuration.

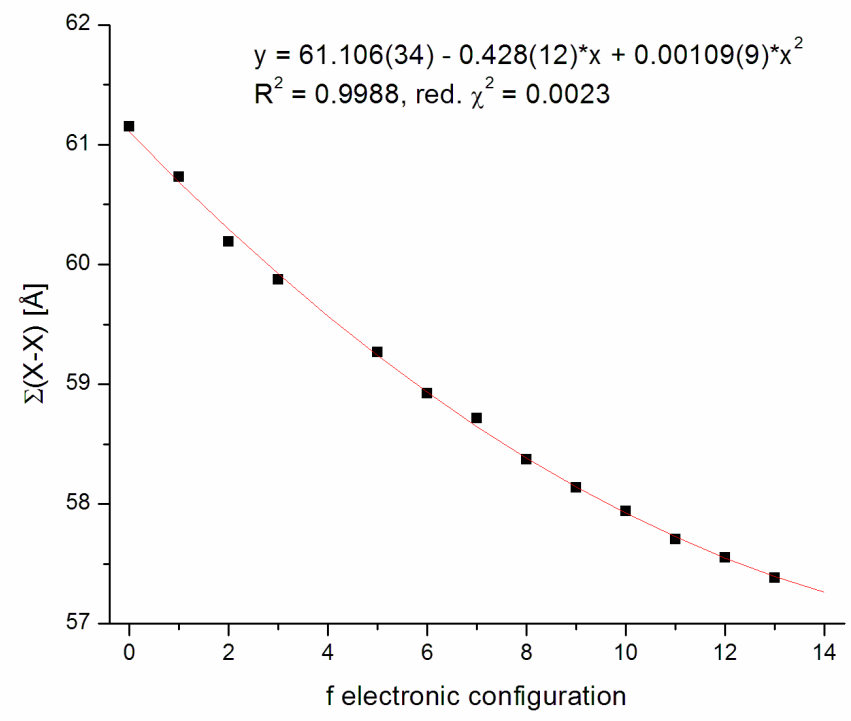

Figure S71. Sum of distance $X-X(X=N, O)$ vs. f electronic configuration. Quadratic fit. 


\section{4. [Ln(PhMeCH-DOTAM) $\left.\left(\mathrm{H}_{2} \mathrm{O}\right)\right](\mathrm{OTf})_{3}$ Complexes $^{9-11}$}

\subsection{Structure}

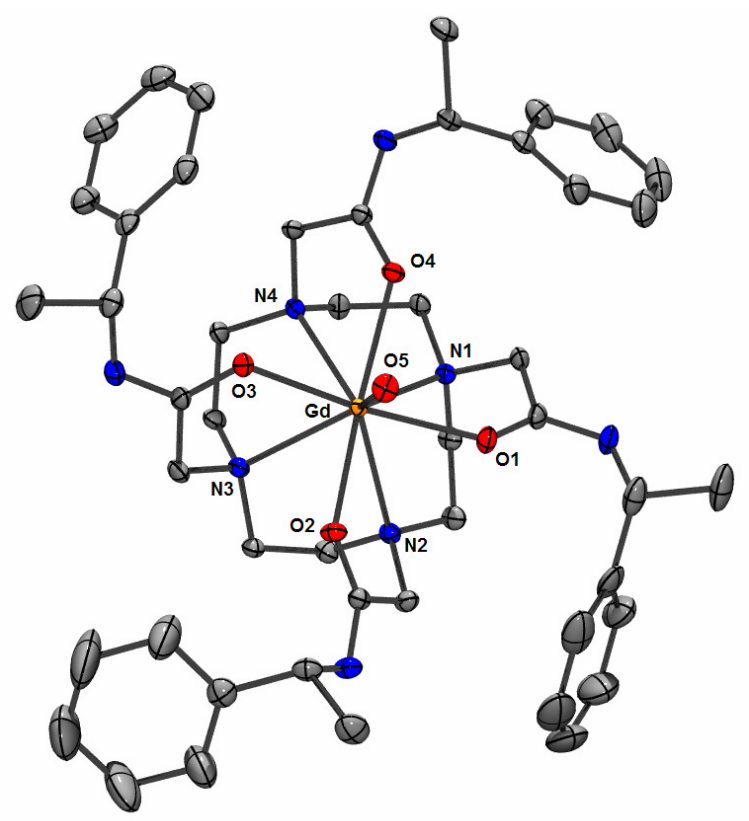

Asymmetric unit of $\left[\mathrm{Ln}(\mathrm{PhMeCH}-\mathrm{DOTAM})\left(\mathrm{H}_{2} \mathrm{O}\right)\right](\mathrm{OTf})_{3}{ }^{*} 3 \mathrm{H}_{2} \mathrm{O}$. ${ }^{9}$ Thermal ellipsoid plot (Ortep 3 for Windows, ${ }^{1} 50 \%$ probability level) with atom numbering scheme.

Hydrogens, triflates, and the three isolated water molecules omitted for clarity.

\subsection{Bond Lengths}

Table S8. Bond lengths in $\left[\mathrm{Ln}(\mathrm{PhMeCH}-\mathrm{DOTAM})\left(\mathrm{H}_{2} \mathrm{O}\right)\right](\mathrm{OTf})_{3}{ }^{*} 3 \mathrm{H}_{2} \mathrm{O} .{ }^{9-11}$

\begin{tabular}{|c|c|c|c|c|c|c|c|c|c|c|}
\hline $\begin{array}{c}\text { f electrons } \\
\text { (Ln) }\end{array}$ & $\begin{array}{c}\mathrm{d}_{\mathrm{Ln}-\mathrm{O} 1}(\mathrm{su}) \\
{[\mathrm{A}]}\end{array}$ & $\begin{array}{c}\mathrm{d}_{\mathrm{Ln}-\mathrm{O} 2}(\mathrm{su}) \\
{[\AA \AA}\end{array}$ & $\begin{array}{c}\mathrm{d}_{\mathrm{Ln}-03}(\mathrm{su}) \\
{[\AA \AA]}\end{array}$ & $\begin{array}{c}d_{L n-04}(s u) \\
{[\AA ̊]}\end{array}$ & $\begin{array}{c}\mathrm{d}_{\mathrm{Ln}-05}(\mathrm{su}) \\
{[\AA \AA \AA}\end{array}$ & $\begin{array}{c}\mathrm{d}_{\mathrm{Ln}-\mathrm{N} 1}(\mathrm{su}) \\
{[\AA \AA]}\end{array}$ & $\begin{array}{c}\mathrm{d}_{\mathrm{Ln}-\mathrm{N} 2}(\mathrm{su}) \\
{[\AA \AA}\end{array}$ & $\begin{array}{c}\mathrm{d}_{\mathrm{Ln-N3}}(\mathrm{su}) \\
{[\AA \AA]}\end{array}$ & $\begin{array}{c}\mathrm{d}_{\mathrm{Ln}-\mathrm{N} 4}(\mathrm{su}) \\
{[\AA \AA]}\end{array}$ & $\begin{array}{c}\Sigma \mathrm{d}_{\mathrm{Ln}-\mathrm{X}}(\mathrm{su}) \\
{[\mathrm{A}]}\end{array}$ \\
\hline $0(\mathrm{La})$ & - & - & - & - & - & - & - & - & - & - \\
\hline $1(\mathrm{Ce})$ & - & - & - & - & - & - & - & - & - & - \\
\hline $2(\operatorname{Pr})^{9}$ & $2.428(2)$ & $2.403(2)$ & $2.434(2)$ & $2.415(2)$ & $2.516(2)$ & $2.696(2)$ & $2.738(2)$ & $2.708(2)$ & $2.746(2)$ & $23.084(18)$ \\
\hline $3(\mathrm{Nd})^{9}$ & $2.414(2)$ & $2.392(2)$ & $2.419(2)$ & $2.402(2)$ & $2.501(2)$ & $2.677(2)$ & $2.715(2)$ & $2.690(2)$ & $2.725(2)$ & $22.935(18)$ \\
\hline $4(\mathrm{Pm})$ & - & - & - & - & - & - & - & - & - & - \\
\hline $5(\mathrm{Sm})^{9}$ & $2.374(4)$ & $2.352(4)$ & $2.383(4)$ & $2.362(4)$ & $2.495(2)$ & $2.647(5)$ & $2.685(5)$ & $2.654(5)$ & $2.697(5)$ & $22.649(40)$ \\
\hline $6(\mathrm{Eu})^{9}$ & $2.377(2)$ & $2.355(2)$ & $2.379(2)$ & $2.361(2)$ & $2.482(2)$ & $2.643(2)$ & $2.680(2)$ & $2.655(2)$ & $2.689(2)$ & $22.621(18)$ \\
\hline $7(\mathrm{Gd})^{9}$ & $2.368(2)$ & $2.346(2)$ & $2.367(2)$ & $2.353(2)$ & $2.460(2)$ & $2.633(2)$ & $2.670(2)$ & $2.650(2)$ & $2.675(2)$ & $22.522(18)$ \\
\hline $8(\mathrm{~Tb})^{10}$ & $2.355(2)$ & $2.333(2)^{*}$ & $2.350(2)^{*}$ & $2.331(2)$ & $2.461(3)$ & $2.624(3)$ & $2.664(3)^{*}$ & $2.642(3)^{*}$ & $2.669(3)$ & $22.429(23)$ \\
\hline $9(\mathrm{Dy})^{9}$ & $2.344(2)$ & $2.315(2)$ & $2.336(2)$ & $2.317(2)$ & $2.454(2)$ & $2.620(2)$ & $2.654(2)$ & $2.644(2)$ & $2.658(2)$ & $22.342(18)$ \\
\hline $10(\mathrm{Ho})$ & - & - & - & - & - & - & - & - & - & - \\
\hline $11(\mathrm{Er})^{10}$ & $2.321(1)$ & $2.296(2)$ & $2.310(1)$ & $2.294(2)$ & 2.432(2) & $2.605(2)$ & $2.639(2)$ & $2.629(2)$ & $2.636(2)$ & $22.162(15)$ \\
\hline $12(\mathrm{Tm})$ & - & - & - & - & - & - & - & - & - & - \\
\hline $13(\mathrm{Yb})^{11}$ & $2.298(2)^{*}$ & $2.274(2)$ & $2.288(2)$ & $2.274(2)^{\star}$ & $2.440(2)$ & $2.598(2)$ & $2.636(2)$ & $2.626(2)$ & $2.630(3)$ & $22.064(19)$ \\
\hline $14(\mathrm{Lu})^{10}$ & $2.292(2)$ & $2.273(2)$ & $2.283(2)$ & $2.273(2)$ & $2.426(2)$ & $2.592(2)$ & $2.636(2)$ & $2.623(2)$ & $2.623(3)$ & $22.021(19)$ \\
\hline $\mathrm{d}_{\mathrm{Pr}} / \mathrm{d}_{\mathrm{Lu}}$ & 1.059 & 1.057 & 1.066 & 1.062 & 1.037 & 1.040 & 1.039 & 1.032 & 1.047 & 1.048 \\
\hline
\end{tabular}

${ }^{*}$ Bond lengths taken from the published cif-files. Incorrectly listed in the original publication (see ref. 10 and 11). 


\subsection{X-X Distances}

Table S9. Distances $\mathrm{X}-\mathrm{X}(\mathrm{X}=\mathrm{N}, \mathrm{O}$ bound to $\mathrm{Ln})$ in [ $\left.\mathrm{Ln}(\mathrm{PhMeCH}-\mathrm{DOTAM})\left(\mathrm{H}_{2} \mathrm{O}\right)\right]$ $(\mathrm{OTf})_{3}{ }^{*} 3 \mathrm{H}_{2} \mathrm{O}$.

\begin{tabular}{|c|c|c|c|c|c|c|c|c|c|c|c|c|c|c|c|c|c|c|c|c|c|}
\hline $\begin{array}{c}\text { f electrons } \\
\text { (Ln) }\end{array}$ & $\begin{array}{c}\mathrm{d}_{\mathrm{O5-01}} \\
{[\AA \AA]}\end{array}$ & $\begin{array}{c}\mathrm{d}_{\mathrm{O5-02}} \\
{[\mathrm{A}]}\end{array}$ & $\begin{array}{c}\mathrm{d}_{\mathrm{O5-03}} \\
{[\AA \AA \AA}\end{array}$ & $\begin{array}{c}d_{05-04} \\
{[\AA \AA]}\end{array}$ & $\begin{array}{c}\mathrm{d}_{\mathrm{O} 1-02} \\
{[\AA \AA]}\end{array}$ & $\begin{array}{c}\mathrm{d}_{\mathrm{O2}-03} \\
{[\AA \AA \AA}\end{array}$ & $\begin{array}{c}\mathrm{d}_{03-04} \\
{[\hat{A}]}\end{array}$ & $\begin{array}{c}\mathrm{d}_{04-01} \\
{[\AA \AA]}\end{array}$ & $\begin{array}{c}\mathrm{d}_{01-\mathrm{N} 1} \\
{[\AA \AA]}\end{array}$ & $\begin{array}{c}\mathrm{d}_{\mathrm{O} 1 \mathrm{-N} 2} \\
{[\AA \AA]}\end{array}$ & $\begin{array}{c}\mathrm{d}_{\mathrm{O} 2 \mathrm{~N} 2} \\
{[\AA \AA]}\end{array}$ & $\begin{array}{c}\mathrm{d}_{\mathrm{O} 2-\mathrm{N} 3} \\
{[\AA \AA \mathrm{A}]}\end{array}$ & $\begin{array}{c}\mathrm{d}_{\text {O3-N3 }} \\
{[\AA \AA \times}\end{array}$ & $\begin{array}{c}\mathrm{d}_{\mathrm{O}-\mathrm{N} 4} \\
{[\AA \AA]}\end{array}$ & $\begin{array}{c}\mathrm{d}_{\mathrm{O}-\mathrm{N} 4} \\
{[\AA \AA \AA}\end{array}$ & $\begin{array}{c}\mathrm{d}_{\mathrm{O}-\mathrm{N} 1} \\
{[\AA \AA \AA}\end{array}$ & $\begin{array}{c}\mathrm{d}_{\mathrm{N} 1-\mathrm{N} 2} \\
{[\AA \AA]}\end{array}$ & $\begin{array}{c}\mathrm{d}_{\mathrm{N2}-\mathrm{N} 3} \\
{[\AA \AA]}\end{array}$ & $\begin{array}{c}\mathrm{d}_{\mathrm{N} 3-\mathrm{N} 4} \\
{[\AA \AA \AA}\end{array}$ & $\begin{array}{c}\mathrm{d}_{\mathrm{N} 4-\mathrm{N} 1} \\
{[\AA \AA \AA}\end{array}$ & $\begin{array}{c}\Sigma \mathrm{d}_{\mathrm{X}-\mathrm{X}} \\
{[\AA \AA]}\end{array}$ \\
\hline 0 (La) & - & - & - & - & - & - & - & - & - & - & - & - & - & - & - & - & - & - & - & - & - \\
\hline $1(\mathrm{Ce})$ & - & - & - & - & - & - & - & - & - & - & - & - & - & - & - & - & - & - & - & - & - \\
\hline $2(\operatorname{Pr})^{9}$ & 2.909 & 2.984 & 3.015 & 2.989 & 3.259 & 3.310 & 3.222 & 3.372 & 2.794 & 3.086 & 2.729 & 3.037 & 2.785 & 3.096 & 2.763 & 3.114 & 2.999 & 3.019 & 2.990 & 3.025 & 60.497 \\
\hline $3(\mathrm{Nd})^{9}$ & 2.883 & 2.955 & 2.967 & 2.953 & 3.236 & 3.276 & 3.209 & 3.337 & 2.791 & 3.067 & 2.725 & 3.018 & 2.779 & 3.082 & 2.764 & 3.087 & 2.988 & 3.009 & 2.979 & 3.013 & 60.118 \\
\hline $4(\mathrm{Pm})$ & - & - & - & - & - & - & - & - & - & - & - & - & - & - & - & - & - & - & - & - & - \\
\hline $5(\mathrm{Sm})^{9}$ & 2.831 & 2.889 & 2.913 & 2.893 & 3.171 & 3.200 & 3.136 & 3.274 & 2.776 & 3.009 & 2.713 & 2.971 & 2.766 & 3.062 & 2.751 & 3.074 & 2.962 & 2.997 & 2.975 & 2.987 & 59.350 \\
\hline $6(\mathrm{Eu})^{9}$ & 2.814 & 2.886 & 2.891 & 2.874 & 3.171 & 3.200 & 3.140 & 3.256 & 2.779 & 3.039 & 2.722 & 2.986 & 2.769 & 3.050 & 2.752 & 3.046 & 2.972 & 2.985 & 2.964 & 2.992 & 59.288 \\
\hline $7(\mathrm{Gd})^{9}$ & 2.793 & 2.861 & 2.858 & 2.844 & 3.154 & 3.176 & 3.133 & 3.236 & 2.776 & 3.024 & 2.724 & 2.980 & 2.770 & 3.038 & 2.751 & 3.028 & 2.965 & 2.980 & 2.953 & 2.984 & 59.028 \\
\hline $8(T b)^{10}$ & 2.781 & 2.847 & 2.838 & 2.823 & 3.129 & 3.153 & 3.101 & 3.211 & 2.770 & 3.006 & 2.720 & 2.970 & 2.767 & 3.025 & 2.745 & 3.010 & 2.962 & 2.980 & 2.950 & 2.981 & 58.769 \\
\hline $9(\mathrm{Dy})^{9}$ & 2.758 & 2.838 & 2.822 & 2.796 & 3.116 & 3.114 & 3.093 & 3.182 & 2.765 & 3.000 & 2.716 & 2.955 & 2.761 & 3.015 & 2.736 & 2.997 & 2.954 & 2.978 & 2.953 & 2.981 & 58.530 \\
\hline $10(\mathrm{Ho})$ & - & - & - & - & - & - & - & - & - & - & - & - & - & - & - & - & - & - & - & - & - \\
\hline $11(\mathrm{Er})^{10}$ & 2.729 & 2.801 & 2.768 & 2.746 & 3.077 & 3.071 & 3.063 & 3.137 & 2.759 & 2.981 & 2.716 & 2.938 & 2.751 & 2.989 & 2.732 & 2.974 & 2.943 & 2.964 & 2.940 & 2.972 & 58.051 \\
\hline $12(\mathrm{Tm})$ & - & - & - & - & - & - & - & - & - & - & - & - & - & - & - & - & - & - & - & - & - \\
\hline $13(\mathrm{Yb})^{11}$ & 2.715 & 2.797 & 2.758 & 2.727 & 3.038 & 3.035 & 3.037 & 3.111 & 2.750 & 2.959 & 2.717 & 2.926 & 2.745 & 2.972 & 2.725 & 2.956 & 2.933 & 2.963 & 2.935 & 2.974 & 57.773 \\
\hline $14(\mathrm{Lu})^{10}$ & 2.704 & 2.777 & 2.736 & 2.710 & 3.032 & 3.022 & 3.033 & 3.101 & 2.747 & 2.954 & 2.727 & 2.921 & 2.742 & 2.963 & 2.733 & 2.953 & 2.930 & 2.966 & 2.931 & 2.974 & 57.656 \\
\hline $\mathrm{d}_{\mathrm{Pr}} / \mathrm{d}_{\mathrm{Lu}}$ & 1.076 & 1.075 & 1.102 & 1.103 & 1.075 & 1.095 & 1.062 & 1.087 & 1.017 & 1.045 & 1.001 & 1.040 & 1.016 & 1.045 & 1.011 & 1.055 & 1.024 & 1.018 & 1.020 & 1.017 & 1.049 \\
\hline
\end{tabular}

\subsection{Trends in Bond Lengths and $X-X$ Distances vs. $f$ Electron Configuration}

Bond Lengths:

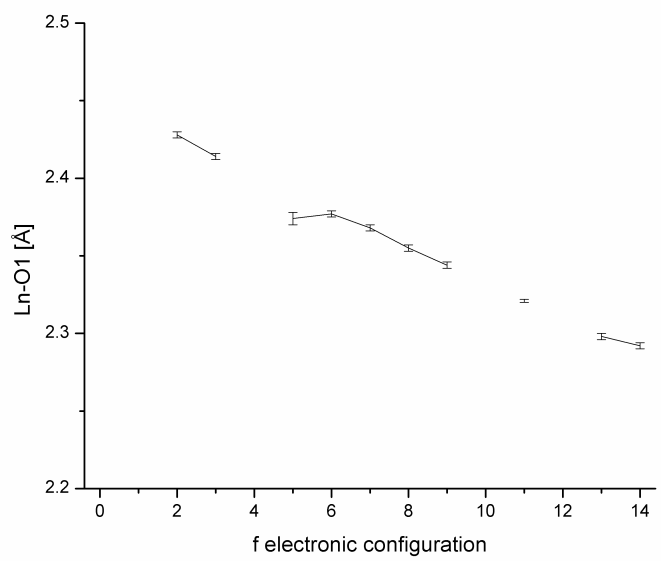

Figure S72. Bond length Ln-O1 vs. f electronic configuration. 


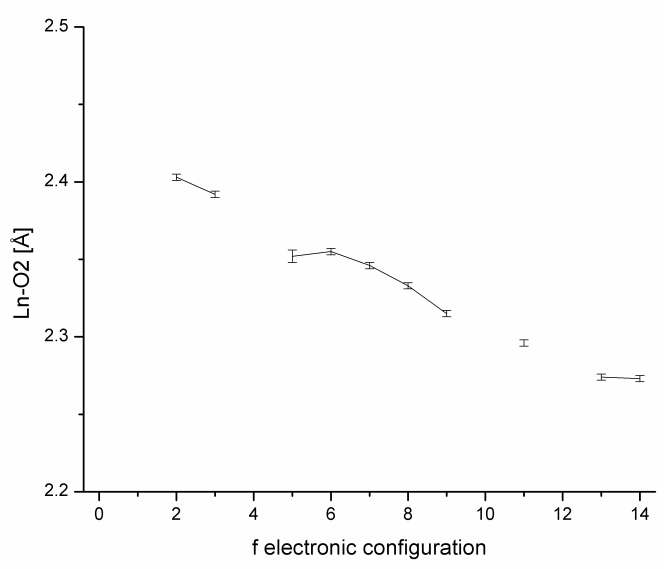

Figure S73. Bond length Ln-O2 vs. f electronic configuration.

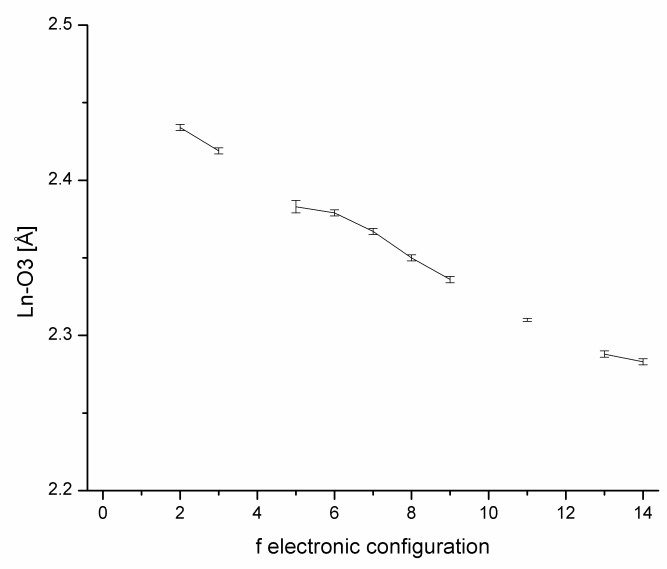

Figure S74. Bond length Ln-O3 vs. f electronic configuration.

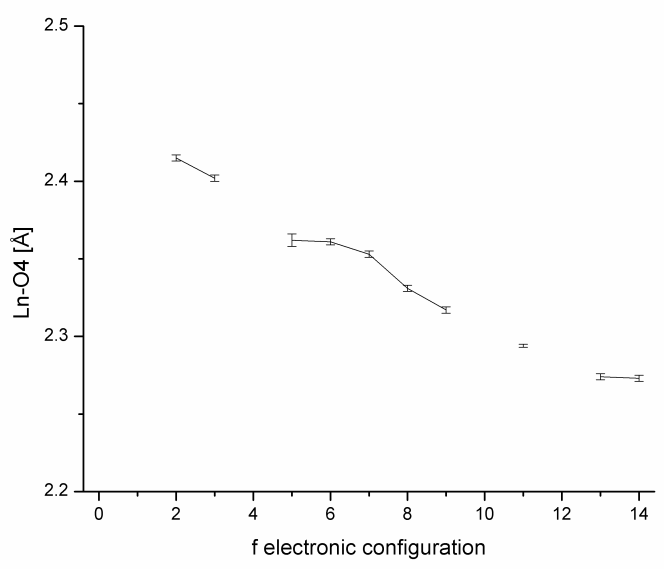

Figure S75. Bond length Ln-O4 vs. f electronic configuration. 


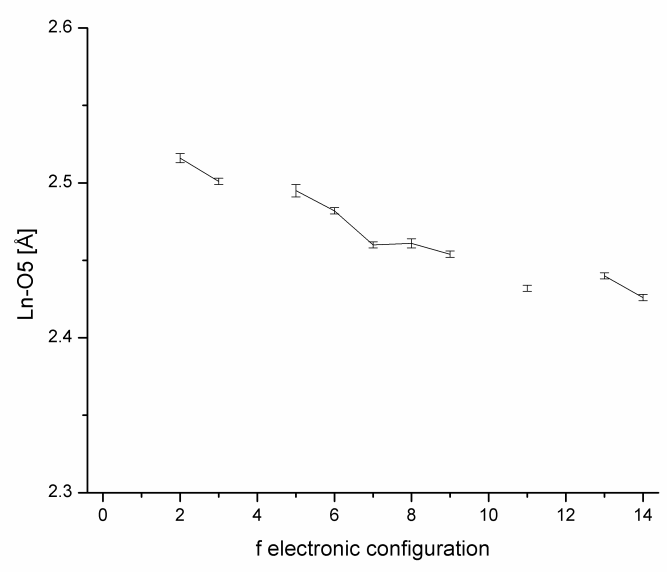

Figure S76. Bond length Ln-O5 vs. f electronic configuration.

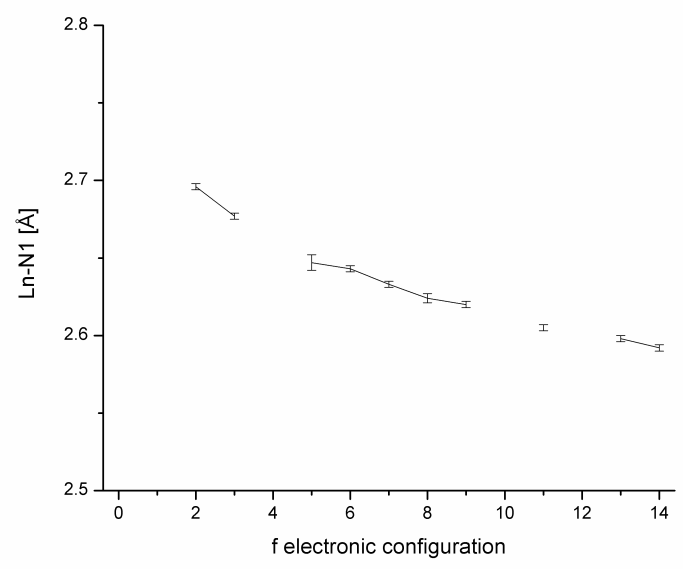

Figure S77. Bond length Ln-N1 vs. f electronic configuration.

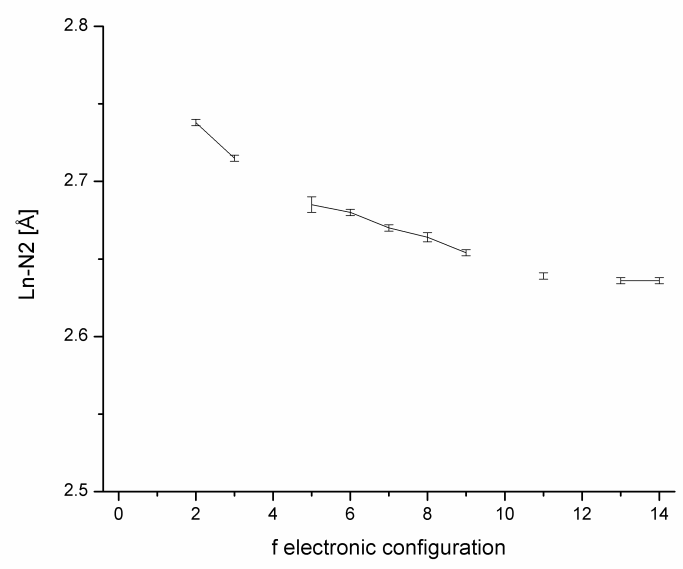

Figure S78. Bond length Ln-N2 vs. f electronic configuration. 


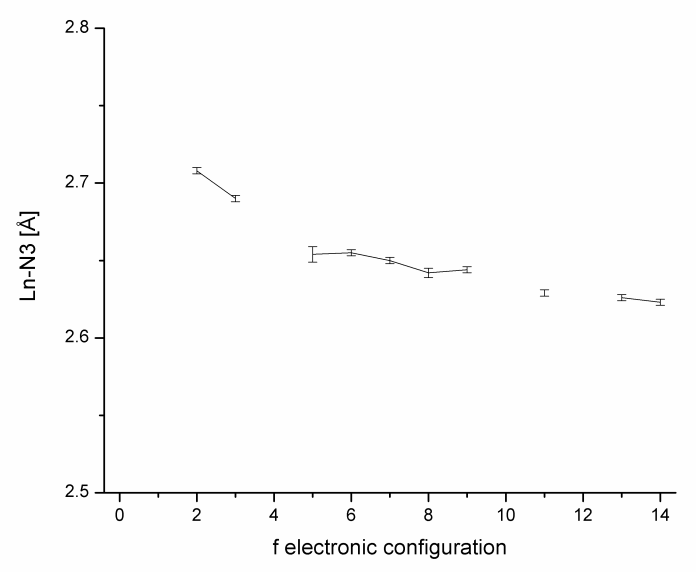

Figure S79. Bond length Ln-N3 vs. f electronic configuration.

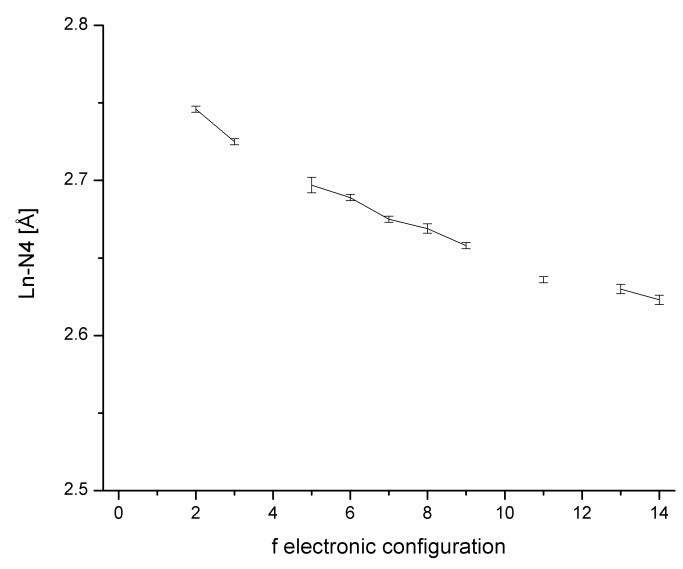

Figure S80. Bond length Ln-N4 vs. f electronic configuration.

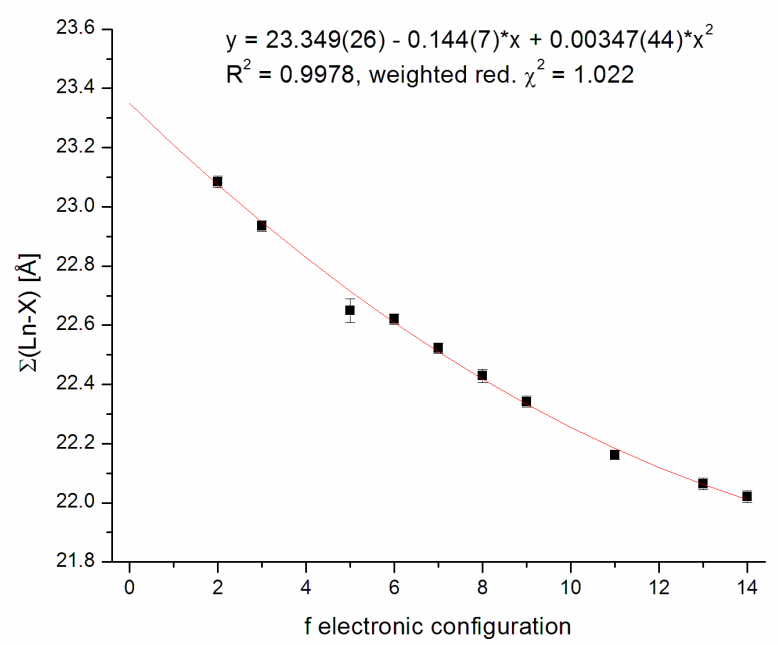

Figure S81. Sum of bond lengths $\operatorname{Ln}-X \operatorname{Ln}-X(X=N, O)$ vs. $f$ electronic configuration. Quadratic fit $\left(\chi^{2}\right.$ - weighting factor: $\left.\mathrm{su}^{-2}\right)$. 
Distances $X-X(X=N, O)$ :

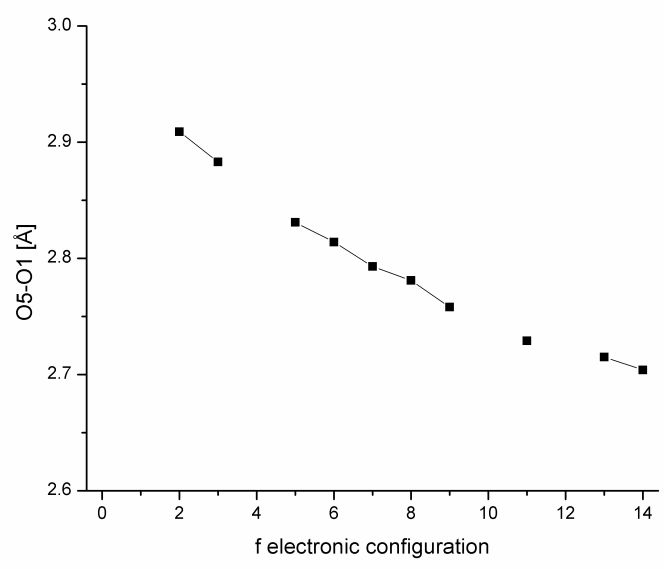

Figure S82. Distance O5-O1 vs. f electronic configuration.

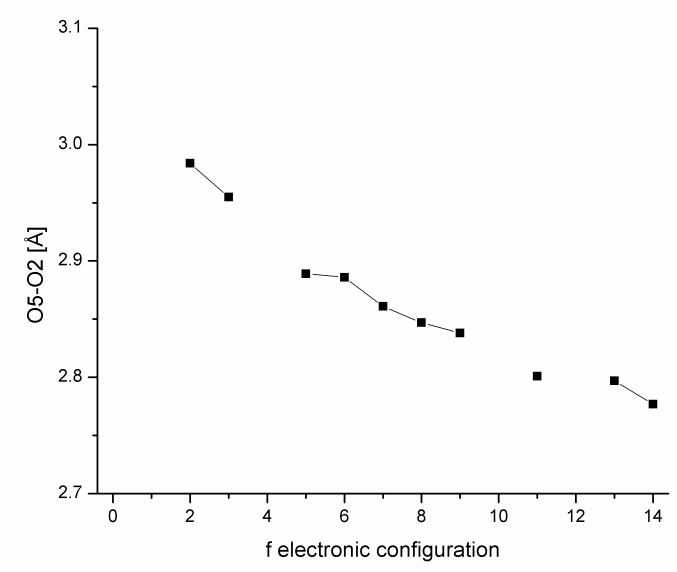

Figure S83. Distance O5-O2 vs. f electronic configuration.

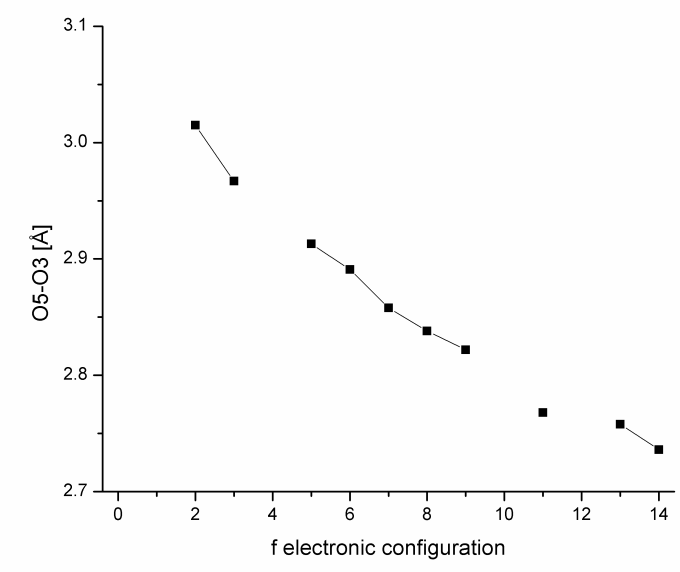

Figure S84. Distance O5-O3 vs. f electronic configuration. 


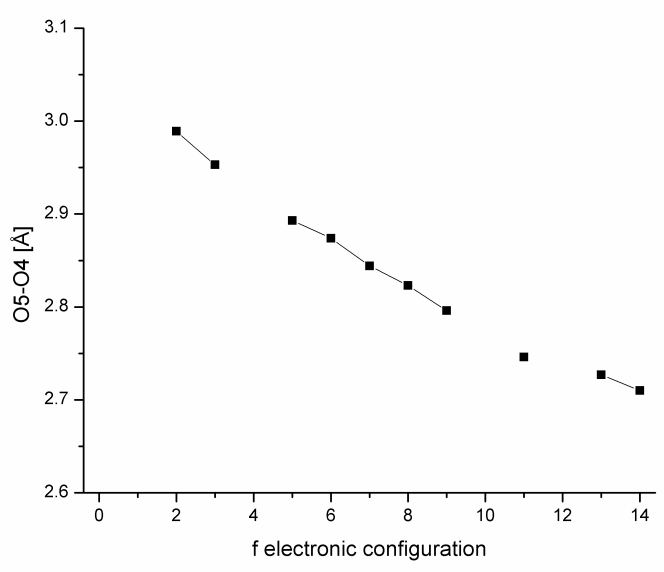

Figure S85. Distance O5-O4 vs. f electronic configuration.

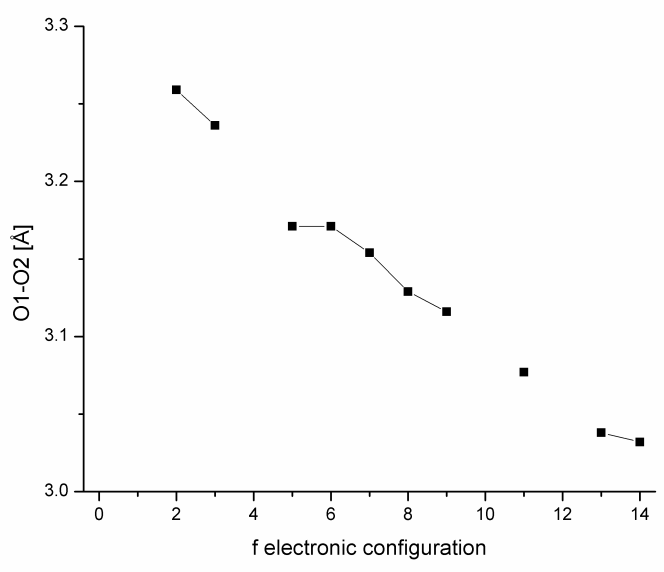

Figure S86. Distance O1-O2 vs. f electronic configuration.

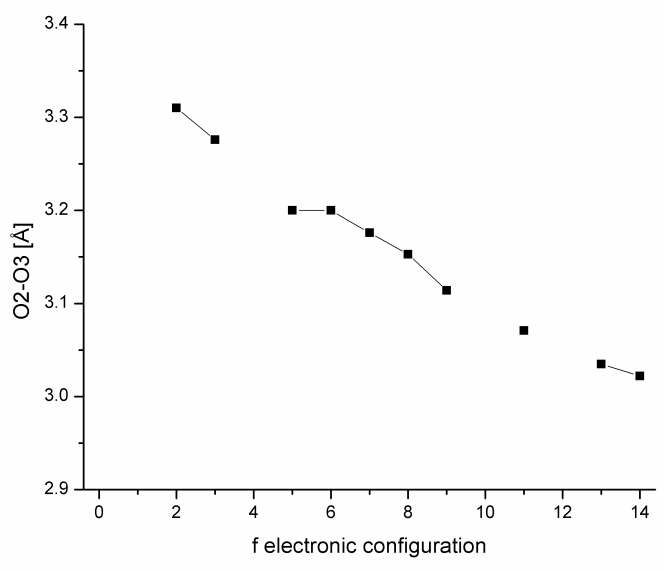

Figure S87. Distance O2-O3 vs. f electronic configuration. 


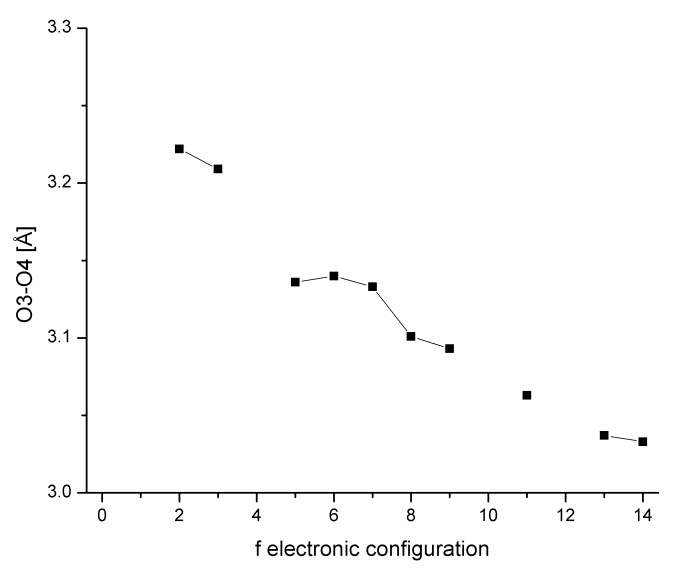

Figure S88. Distance O3-O4 vs. f electronic configuration.

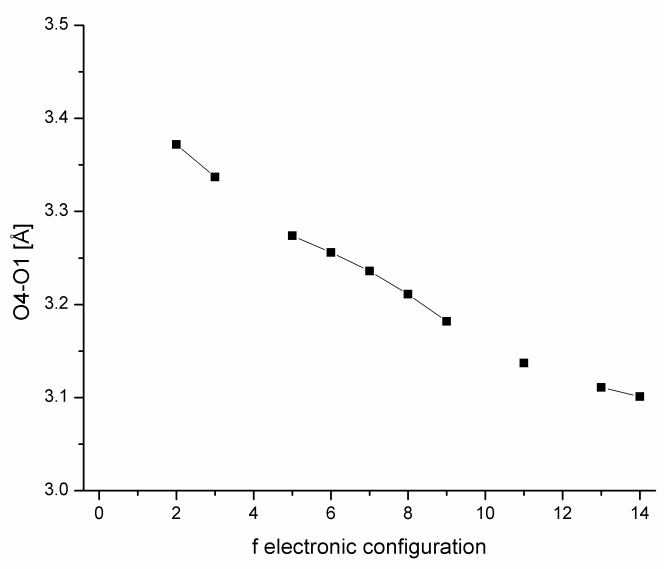

Figure S89. Distance O4-O1 vs. f electronic configuration.

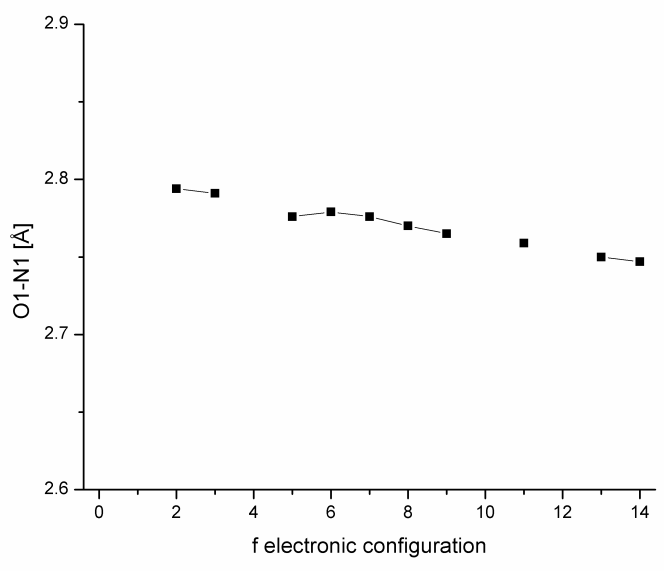

Figure S90. Distance O1-N1 vs. f electronic configuration. 


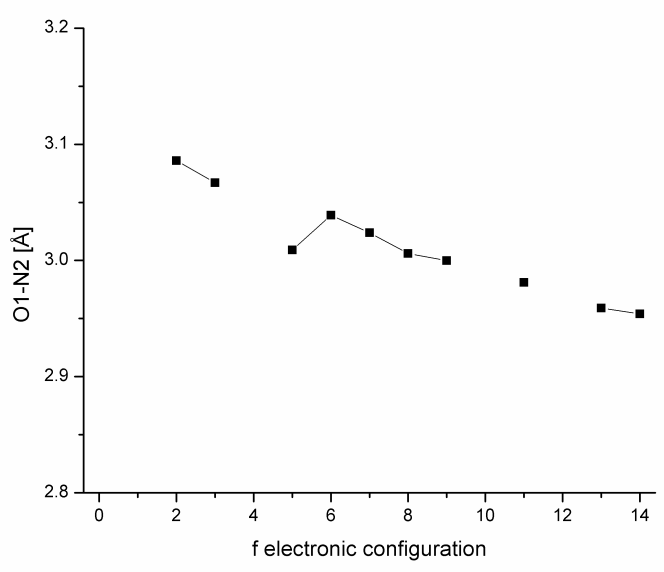

Figure S91. Distance O1-N2 vs. f electronic configuration.

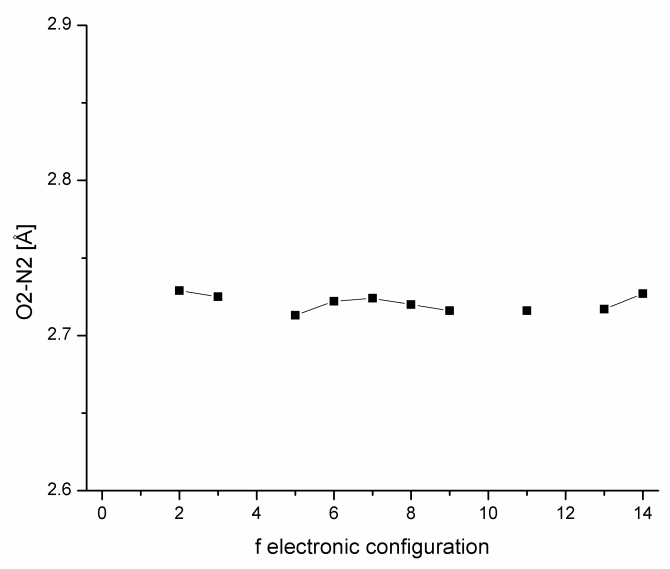

Figure S92. Distance O2-N2 vs. f electronic configuration.

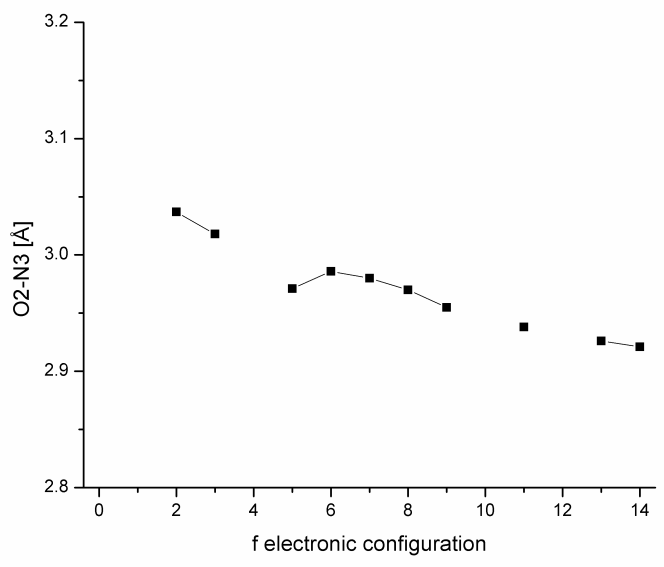

Figure S93. Distance O2-N3 vs. f electronic configuration. 


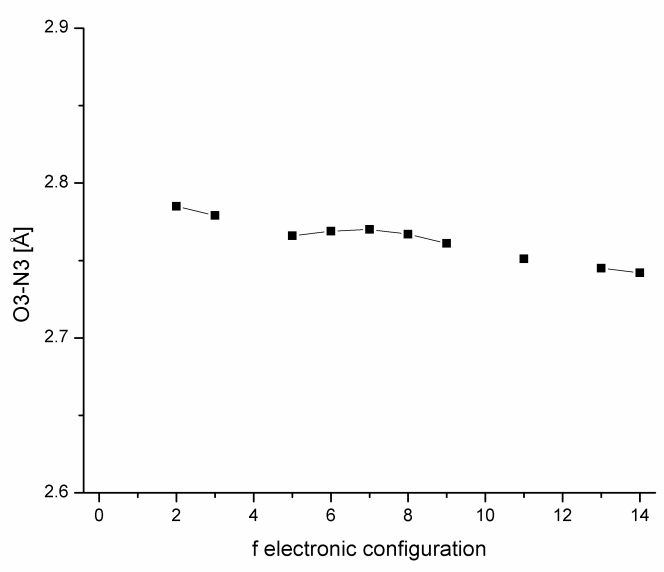

Figure S94. Distance O3-N3 vs. f electronic configuration.

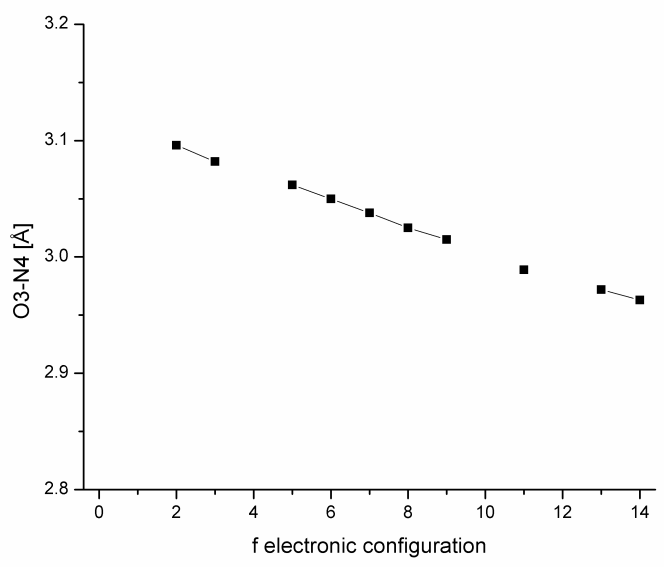

Figure S95. Distance O3-N4 vs. f electronic configuration.

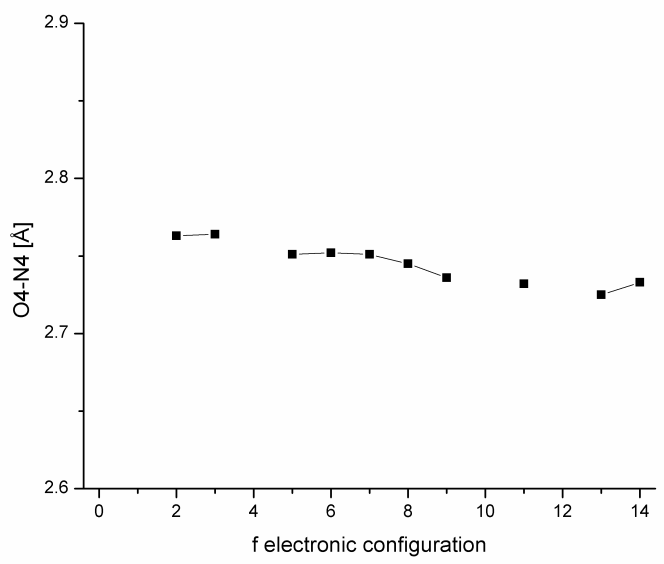

Figure S96. Distance O4-N4 vs. f electronic configuration. 


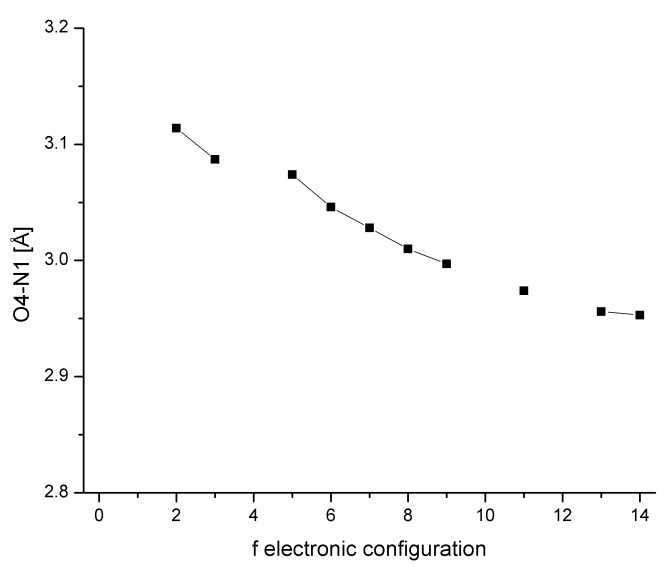

Figure S97. Distance O4-N1 vs. f electronic configuration.

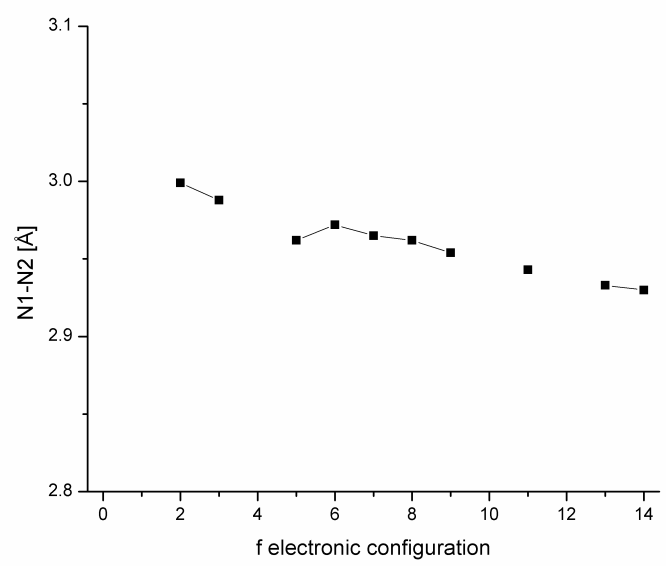

Figure S98. Distance N1-N2 vs. f electronic configuration.

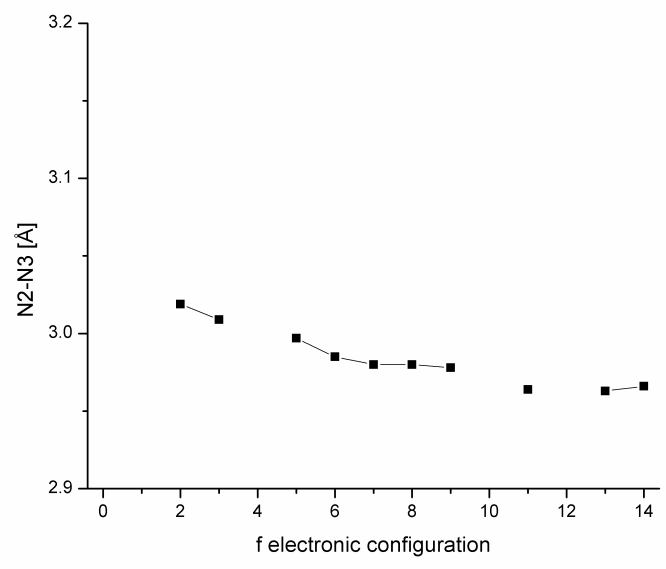

Figure S99. Distance N2-N3 vs. f electronic configuration. 


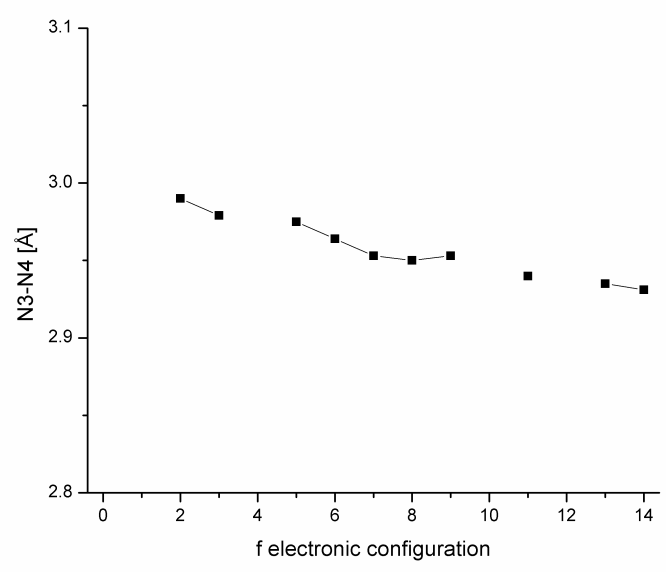

Figure S100. Distance N3-N4 vs. f electronic configuration.

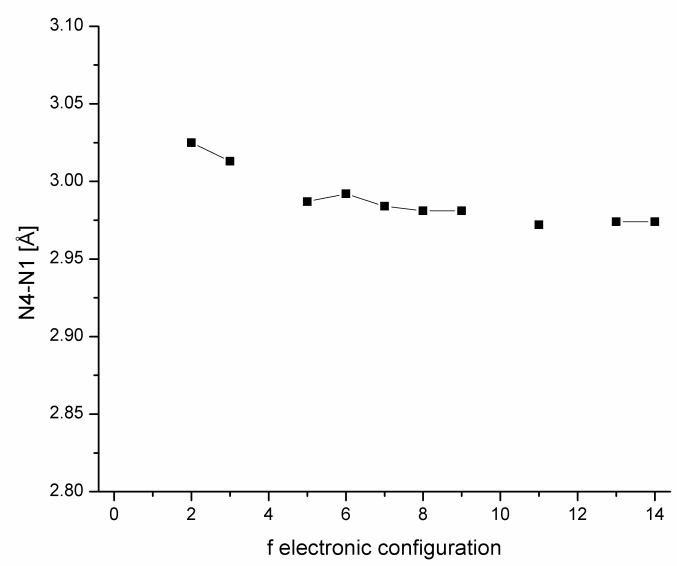

Figure S101. Distance N4-N1 vs. f electronic configuration.

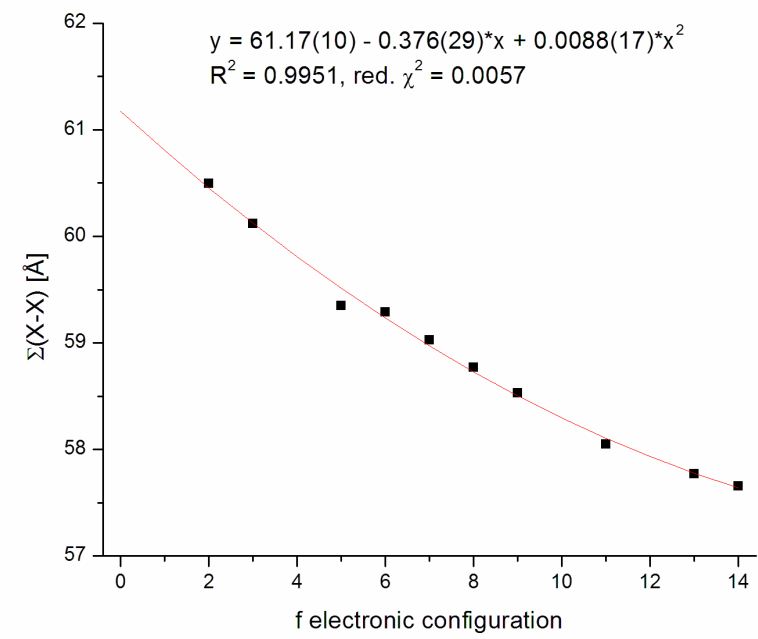

Figure S102. Sum of distance $X-X(X=N, O)$ vs. f electronic configuration. Quadratic fit. 


\section{$5 \quad\left[\mathrm{Ln}\left(\mathrm{H}_{2} \mathrm{O}\right)_{9}\right]\left(\mathrm{EtOSO}_{3}\right)_{3}$ Complexes $^{12}$}

\subsection{Bond Lengths}

Table S10. Bond lengths in $\left[\mathrm{Ln}\left(\mathrm{H}_{2} \mathrm{O}\right)_{9}\right]\left(\mathrm{EtOSO}_{3}\right)_{3} \cdot{ }^{12}$

\begin{tabular}{|c|c|c|c|}
\hline f electrons (Ln) & $\mathrm{d}_{\mathrm{Ln}-01}(\mathrm{su})[\hat{\mathrm{A}}](6 \mathrm{x})$ & $\mathrm{d}_{\mathrm{Ln}-02}(\mathrm{su})[\dot{\mathrm{A}}](3 \mathrm{x})$ & $\Sigma \mathrm{d}_{\mathrm{Ln}-\mathrm{O}}(\mathrm{su})[\AA]$ \\
\hline 0 (La) & $2.517(1)$ & $2.616(3)$ & $22.950(15)$ \\
\hline $1(\mathrm{Ce})$ & $2.491(1)$ & $2.600(3)$ & $22.746(15)$ \\
\hline $2(\operatorname{Pr})$ & $2.470(2)$ & $2.583(3)$ & $22.569(21)$ \\
\hline $3(\mathrm{Nd})$ & $2.457(1)$ & $2.570(2)$ & $22.452(12)$ \\
\hline $4(\mathrm{Pm})$ & - & - & \\
\hline $5(\mathrm{Sm})$ & $2.430(1)$ & $2.550(4)$ & $22.230(18)$ \\
\hline $6(\mathrm{Eu})$ & $2.415(1)$ & $2.542(3)$ & 22.116(15) \\
\hline $7(\mathrm{Gd})$ & $2.401(1)$ & $2.536(3)$ & $22.014(15)$ \\
\hline $8(\mathrm{~Tb})$ & $2.382(2)$ & $2.527(3)$ & $21.873(21)$ \\
\hline 9 (Dy) & $2.371(1)$ & $2.517(4)$ & $21.777(15)$ \\
\hline $10(\mathrm{Ho})$ & $2.362(2)$ & $2.511(4)$ & $21.705(24)$ \\
\hline $11(\mathrm{Er})$ & $2.352(2)$ & $2.503(3)$ & $21.621(21)$ \\
\hline $12(\mathrm{Tm})$ & $2.340(2)$ & $2.504(5)$ & $21.552(27)$ \\
\hline $13(\mathrm{Yb})$ & $2.324(2)$ & $2.503(3)$ & $21.453(21)$ \\
\hline $14(\mathrm{Lu})$ & $2.318(2)$ & $2.497(3)$ & $21.399(21)$ \\
\hline$\overline{d_{L a} / d_{L u}}$ & 1.086 & 1.048 & 1.072 \\
\hline
\end{tabular}

\subsection{O-O Distances}

Table S11. Distances O-O (O bound to $\mathrm{Ln})$ in $\left[\mathrm{Ln}\left(\mathrm{H}_{2} \mathrm{O}\right)_{9}\right]\left(\mathrm{EtOSO}_{3}\right)_{3}{ }^{12}$

\begin{tabular}{|c|c|c|c|c|c|}
\hline f electrons (Ln) & $\mathrm{d}_{\mathrm{O} 1^{\prime}-\mathrm{O} 2}[\AA](6 \mathrm{x})$ & 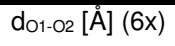 & 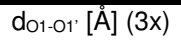 & $\mathrm{d}_{\mathrm{O} 1^{\prime}-\mathrm{O}{ }^{\prime}}[\dot{\mathrm{A}}](6 \mathrm{x})^{*}$ & $\Sigma \mathrm{d}_{\mathrm{O}-\mathrm{O}}[\AA]$ \\
\hline $0(\mathrm{La})$ & 2.770 & 3.058 & 3.531 & 3.108 & 64.209 \\
\hline $1(\mathrm{Ce})$ & 2.753 & 3.034 & 3.509 & 3.062 & 63.621 \\
\hline $2(\mathrm{Pr})$ & 2.735 & 3.009 & 3.483 & 3.033 & 63.111 \\
\hline $3(\mathrm{Nd})$ & 2.726 & 2.995 & 3.475 & 3.010 & 62.811 \\
\hline $4(\mathrm{Pm})$ & - & - & - & - & - \\
\hline $5(\mathrm{Sm})$ & 2.704 & 2.966 & 3.444 & 2.969 & 62.166 \\
\hline $6(\mathrm{Eu})$ & 2.695 & 2.952 & 3.428 & 2.948 & 61.854 \\
\hline $7(\mathrm{Gd})$ & 2.683 & 2.940 & 3.402 & 2.935 & 61.554 \\
\hline $8(\mathrm{~Tb})$ & 2.673 & 2.920 & 3.379 & 2.909 & 61.149 \\
\hline 9 (Dy) & 2.661 & 2.909 & 3.369 & 2.890 & 60.867 \\
\hline $10(\mathrm{Ho})$ & 2.654 & 2.901 & 3.357 & 2.878 & 60.669 \\
\hline $11(\mathrm{Er})$ & 2.649 & 2.884 & 3.339 & 2.868 & 60.423 \\
\hline $12(\mathrm{Tm})$ & 2.648 & 2.875 & 3.325 & 2.853 & 60.231 \\
\hline $13(\mathrm{Yb})$ & 2.638 & 2.865 & 3.300 & 2.833 & 59.916 \\
\hline $14(\mathrm{Lu})$ & 2.633 & 2.854 & 3.285 & 2.832 & 59.769 \\
\hline $\mathrm{d}_{\mathrm{La}} / \mathrm{d}_{\mathrm{Lu}}$ & 1.052 & 1.071 & 1.075 & 1.097 & 1.074 \\
\hline
\end{tabular}

* Distance not listed in the original publication; values calculated from the published coordinates (see ref. 12). 


\subsection{Trends in Bond Lengths and O-O Distances vs. $\mathrm{f}$ Electron Configuration}

Bond Lengths:

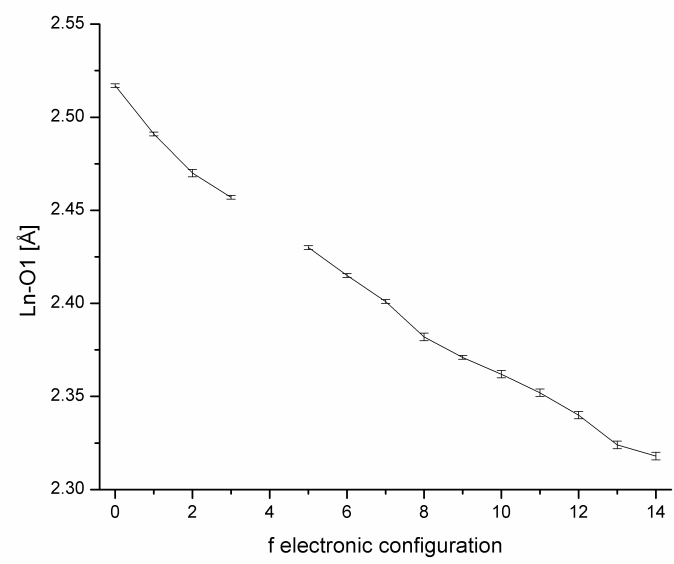

Figure S103. Bond length Ln-O1 vs. f electronic configuration.

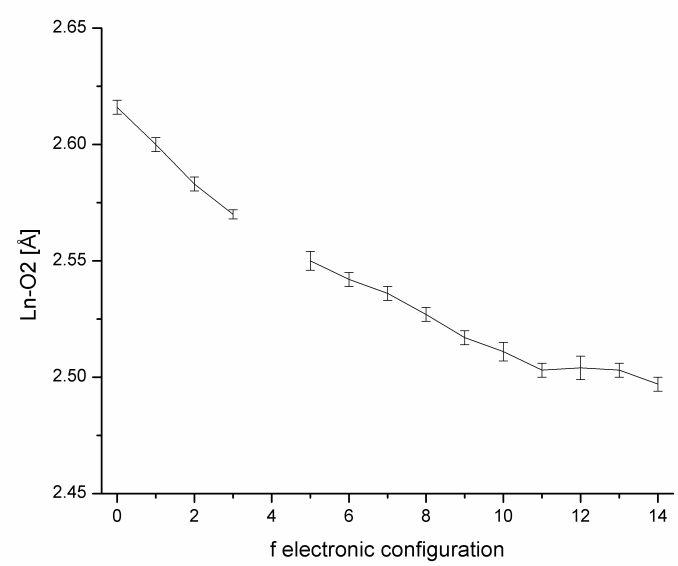

Figure S104. Bond length Ln-O2 vs. f electronic configuration.

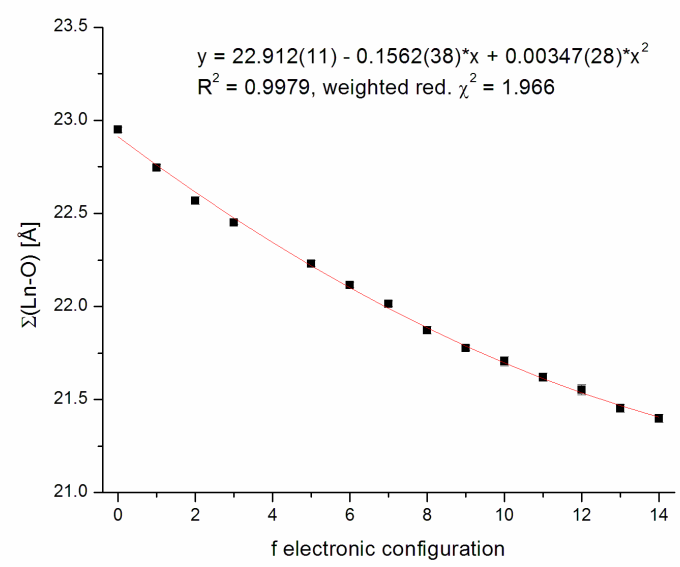

Figure S105. Sum of bond lengths Ln-O vs. f electronic configuration. Quadratic fit $\left(\chi^{2}\right.$ - weighting factor: $\left.\mathrm{su}^{-2}\right)$. 


\section{Distances O-O:}

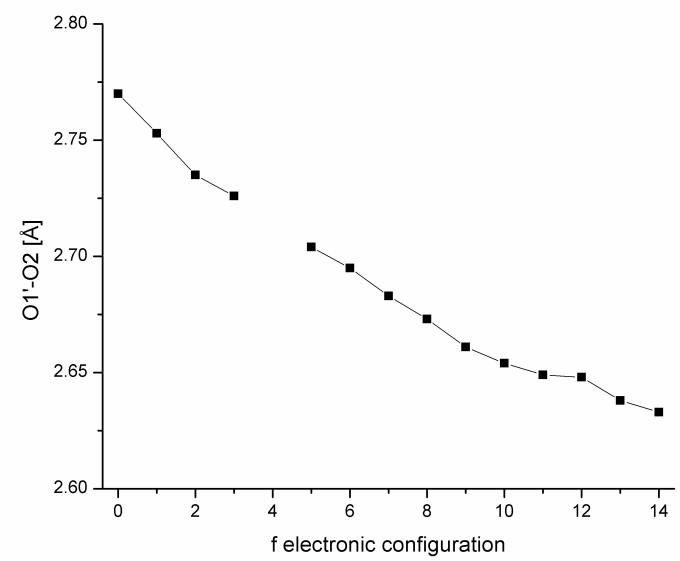

Figure S106. Distance O1'-O2 vs. f electronic configuration.

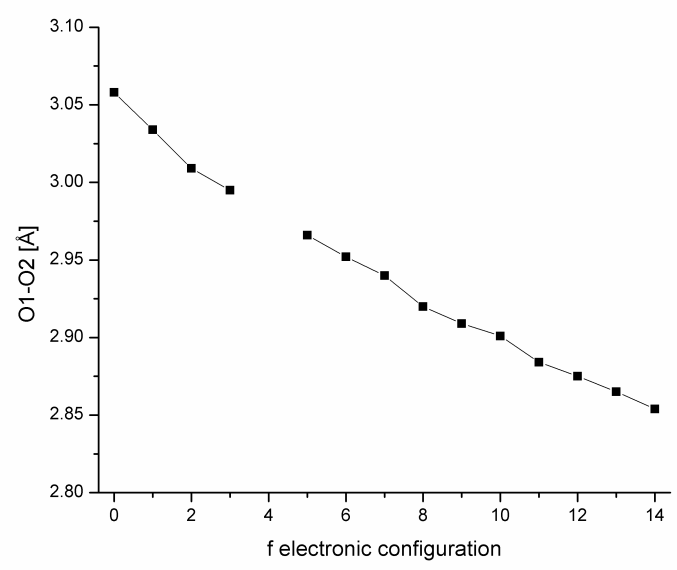

Figure S107. Distance O1-O2 vs. f electronic configuration.

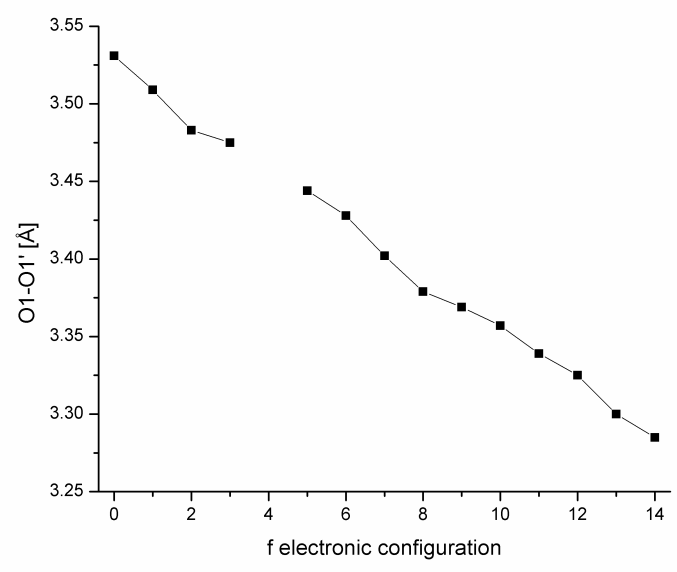

Figure S108. Distance O1-O1'vs. f electronic configuration. 


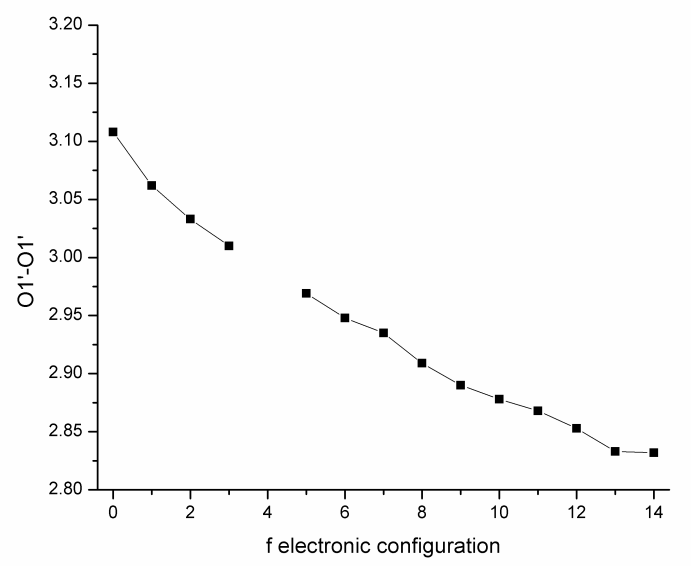

Figure S109. Distance O1'-O1'vs. f electronic configuration.

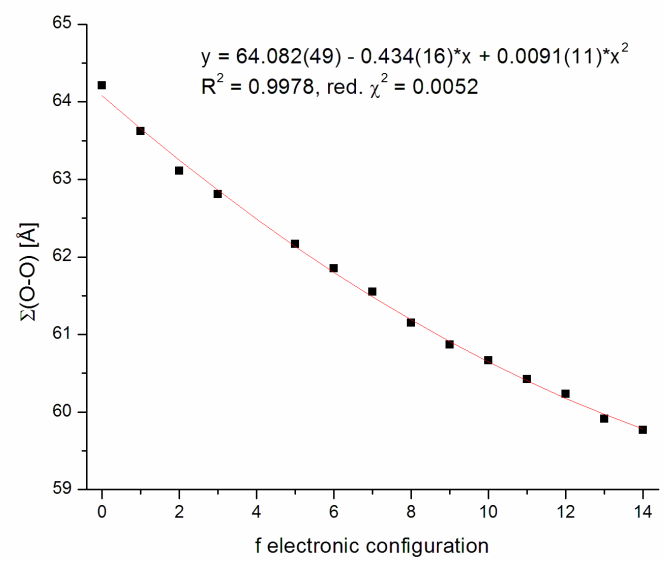

Figure S110. Sum of distances O-O vs. f electronic configuration. Quadratic fit $\left(\chi^{2}\right.$ - weighting factor: $\left.\mathrm{su}^{-2}\right)$. 


\section{The Average X-X Distance vs. the Average Ln-X Bond Length}

Table S12. Average distances Ln-X and X-X (X=N,O; bound to Ln).

\begin{tabular}{|c|c|c|c|c|c|c|c|c|c|c|}
\hline $\begin{array}{c}\text { f electrons } \\
\text { (Ln) }\end{array}$ & $\begin{array}{r}\text { [Ln(TREN-1,2- } \\
\left.\text { HOIQO) }\left(\mathrm{H}_{2} \mathrm{O}\right)\right] \\
\text { avg. Ln-O }[\hat{A}]\end{array}$ & $\begin{array}{c}\text { [Ln(TREN-1,2- } \\
\left.\text { HOIQO) }\left(\mathrm{H}_{2} \mathrm{O}\right)\right] \\
\text { avg. O-O }[\hat{\mathrm{A}}]\end{array}$ & $\begin{array}{l}{\left[\mathrm{Ln} \text { (tptz) }\left(\mathrm{NO}_{3}\right)_{3}\right.} \\
\left.\quad\left(\mathrm{H}_{2} \mathrm{O}\right)\right] \\
\text { avg. } \mathrm{Ln}-\mathrm{X}[\mathrm{A}]\end{array}$ & $\begin{array}{c}{\left[\mathrm{Ln}(\mathrm{tptz})\left(\mathrm{NO}_{3}\right)_{3}\right.} \\
\left.\left(\mathrm{H}_{2} \mathrm{O}\right)\right] \\
\text { avg. X-X } \mathrm{X}[\AA]\end{array}$ & $\begin{array}{c}{[\mathrm{Ln}((\mathrm{PhMeCH}-} \\
\left.\text { DOTAM) }\left(\mathrm{H}_{2} \mathrm{O}\right)\right](\mathrm{OTf})_{3} \\
\text { avg. } \mathrm{Ln}-\mathrm{X}[\dot{\mathrm{A}}]\end{array}$ & $\begin{array}{c}{[\mathrm{Ln}((\mathrm{PhMeCH}-} \\
\left.\text { DOTAM) }\left(\mathrm{H}_{2} \mathrm{O}\right)\right](\mathrm{OTf})_{3} \\
\text { avg. X-X }[\AA \AA A]\end{array}$ & 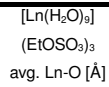 & 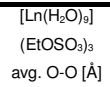 & 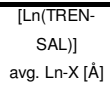 & $\begin{array}{c}\text { [Ln(TREN- } \\
\text { SAL)] } \\
\text { avg. X-X }[\dot{A}]\end{array}$ \\
\hline 0 (La) & 2.486 & 3.152 & 2.615 & 2.912 & - & - & 2.550 & 3.058 & - & - \\
\hline $2(\mathrm{Pr})$ & 2.448 & 3.104 & 2.575 & 2.866 & 2.565 & 3.025 & 2.508 & 3.005 & 2.558 & 3.322 \\
\hline $3(\mathrm{Nd})$ & 2.431 & 3.083 & 2.562 & 2.851 & 2.548 & 3.006 & 2.495 & 2.991 & 2.547 & 3.316 \\
\hline $4(\mathrm{Pm})$ & - & - & - & - & - & - & - & - & - & - \\
\hline $7(\mathrm{Gd})$ & 2.381 & 3.017 & 2.513 & 2.796 & 2.502 & 2.951 & 2.446 & 2.931 & 2.496 & 3.250 \\
\hline $8(\mathrm{~Tb})$ & 2.362 & 2.993 & 2.498 & 2.780 & 2.492 & 2.938 & 2.430 & 2.912 & 2.478 & 3.223 \\
\hline 9 (Dy) & 2.355 & 2.984 & 2.488 & 2.769 & 2.482 & 2.927 & 2.420 & 2.898 & 2.473 & 3.212 \\
\hline $10(\mathrm{Ho})$ & 2.340 & 2.965 & 2.480 & 2.759 & - & - & 2.412 & 2.889 & 2.456 & 3.198 \\
\hline $11(\mathrm{Er})$ & 2.334 & 2.957 & 2.470 & 2.748 & 2.462 & 2.903 & 2.402 & 2.877 & - & - \\
\hline $12(\mathrm{Tm})$ & 2.324 & 2.947 & 2.465 & 2.741 & - & - & 2.395 & 2.868 & - & - \\
\hline
\end{tabular}

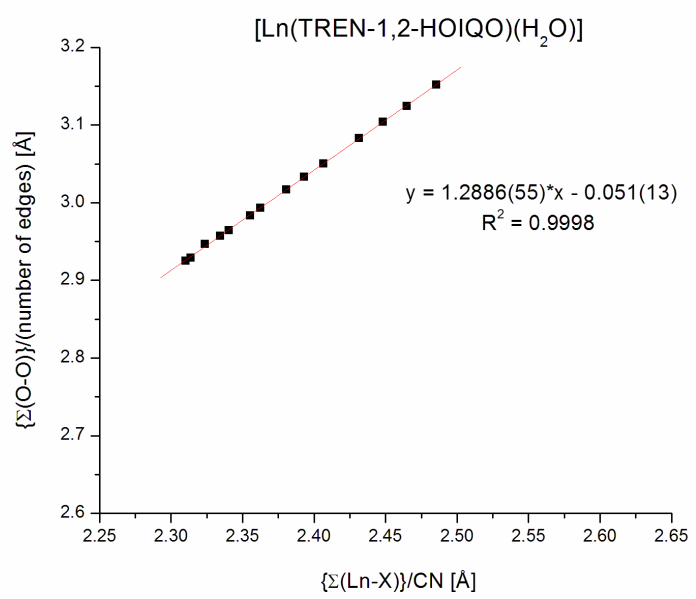

Figure S111. Average O-O vs. average $\mathrm{Ln}-\mathrm{O}$ in $\left[\mathrm{Ln}(\mathrm{TREN}-1,2-\mathrm{HOIQO})\left(\mathrm{H}_{2} \mathrm{O}\right)\right]$.

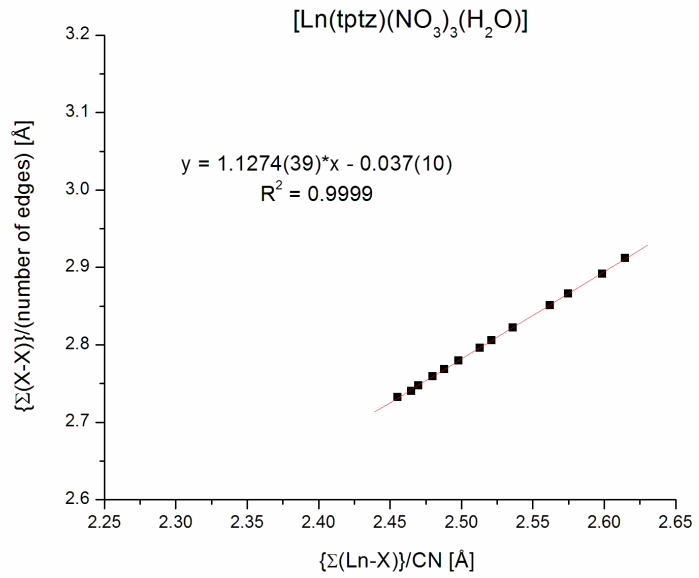

Figure $\mathrm{S} 112$. Average O-O vs. average $\mathrm{Ln}-\mathrm{O}$ in $\left[\mathrm{Ln}(\mathrm{tptz})\left(\mathrm{NO}_{3}\right)_{3}\left(\mathrm{H}_{2} \mathrm{O}\right)\right]$. 


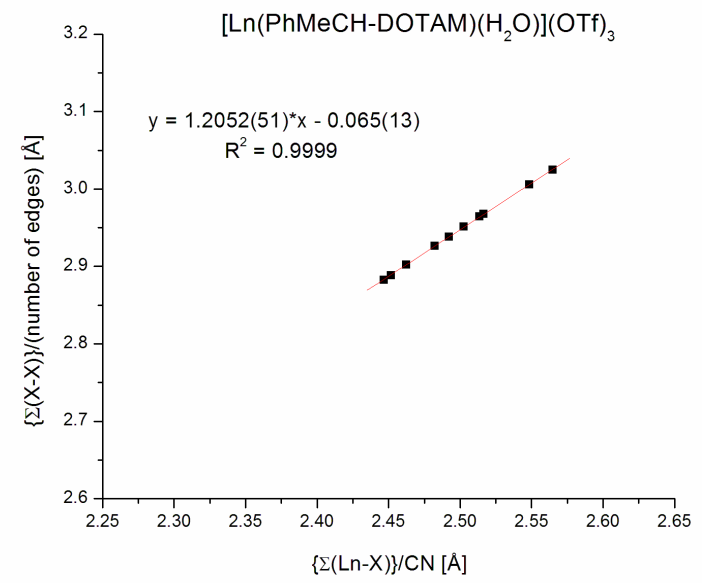

Figure S113. Average O-O vs. average $\mathrm{Ln}-\mathrm{O}$ in $[\mathrm{Ln}(\mathrm{PhMeCH}-\mathrm{DOTAM})$ $\left.\left(\mathrm{H}_{2} \mathrm{O}\right)\right](\mathrm{OTf})_{3}$.

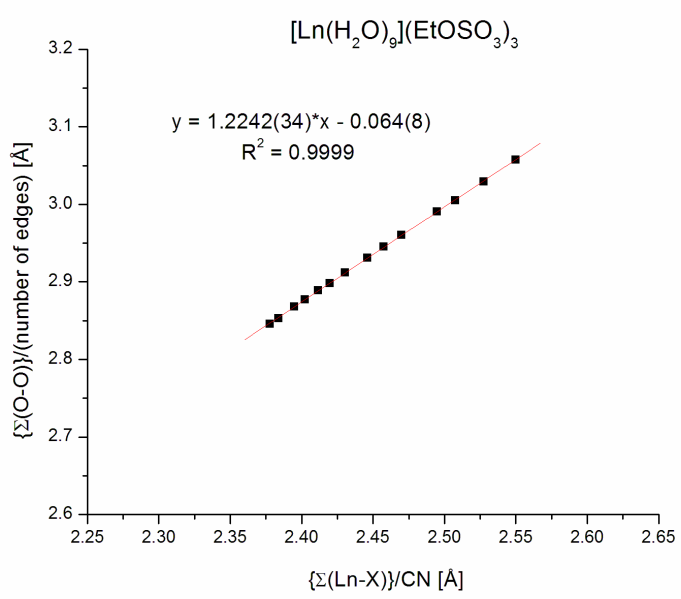

Figure S114. Average O-O vs. average $\mathrm{Ln}-\mathrm{O}$ in $\left[\mathrm{Ln}\left(\mathrm{H}_{2} \mathrm{O}\right)_{9}\right]\left(\mathrm{EtOSO}_{3}\right)_{3}$.

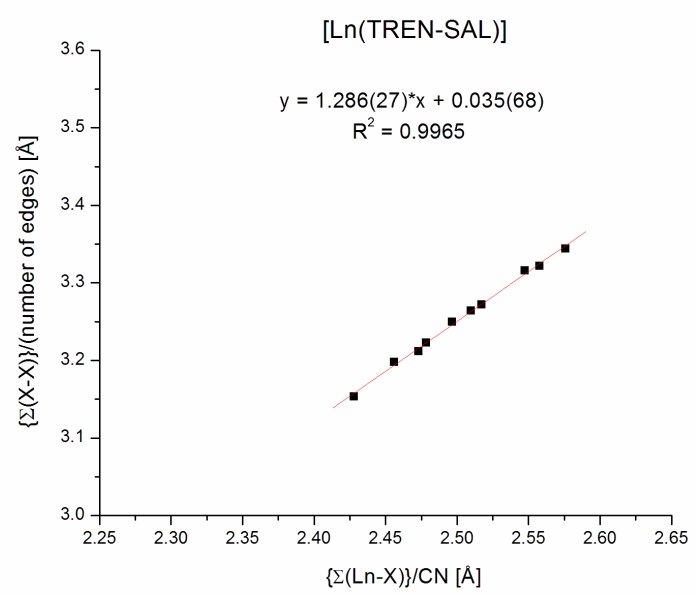

Figure S115. Average X-X vs. average $\operatorname{Ln}-\mathrm{X}$ in [Ln(TREN-SAL)]. 


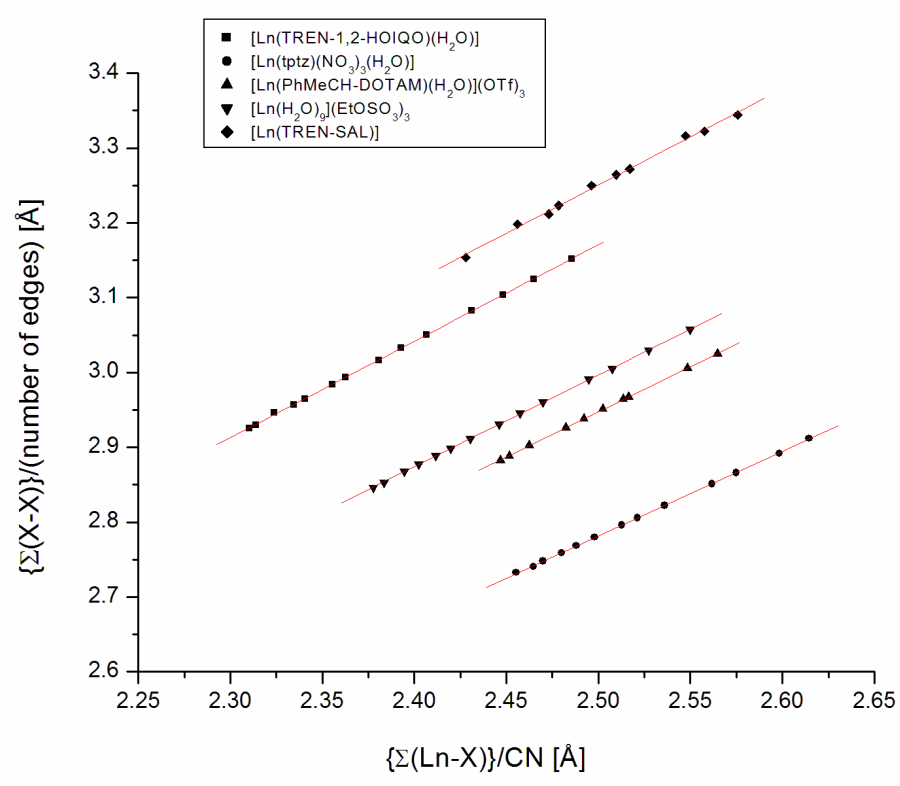

Figure S116. Average $X-X$ vs. average $L n-X$ in all complexes. 


\section{References}

1 ORTEP-3 for Windows: Farrugia, L.J. J. Appl. Cryst. 1997, 30, 565.

2 Seitz, M.; Pluth, M.D.; Raymond, K.N. Inorg. Chem. 2007, 46, 351353.

3 For the definition of the shape measure $S$ see: Xu, J.; Radkov, E.; Ziegler, M.; Raymond, K.N. Inorg. Chem. 2000, 39, 4156-4164.

4 Bernhardt, P.V.; Flanagan, B.M.; Riley, M.J.; Aust. J. Chem. 2001, 54, 229-232.

5 Bernhardt, P.V.; Flanagan, B.M.; Riley, M.J.; Aust. J. Chem. 2000, 53, 229-231.

6 Kanesato, M.; Yokoyama, T.; Anal. Sci. 2000, 16, 335-336.

7 Kanesato, M.; Yokoyama, T.; Chem. Lett. 1999, 137-138.

8 Cotton, S.A.; Franckevicius, V.; Mahon, M.F.; Ooi, L.L.; Raithby, P.R.; Teat, S.J. Polyhedron 2006, 25, 1057-1068.

9 Parker, D.; Puschmann, H.; Batsanov, A.S.; Senanayake, K. Inorg. Chem. 2003, 42, 8646-8651.

10 Aime, S.; Barge, A.; Batsanov, A.S.; Botta, M.; Delli Castelli, D.; Fedeli, F.; Mortillaro, A.; Parker, D.; Puschmann, H. Chem. Commun. 2002, 1120-1121.

11 Batsanov, A.S.; Beeby, A.; Bruce, J.I.; Howard, J.A.K.; Kenwright, A.M.; Parker, D. Chem. Commun. 1999, 1011-1012.

12 Gerkin, R.E.; Reppart, W.J. Acta Crystallogr. C 1984, 40, 781-786. 